\title{
CONVERGENCE OF A BOUNDARY INTEGRAL METHOD FOR WATER WAVES*
}

\author{
J. THOMAS BEALE ${ }^{\dagger}$, THOMAS Y. HOU ${ }^{\ddagger}$, AND JOHN LOWENGRUB $\$$
}

\begin{abstract}
We prove nonlinear stability and convergence of certain boundary integral methods for time-dependent water waves in a two-dimensional, inviscid, irrotational, incompressible fluid, with or without surface tension. The methods are convergent as long as the underlying solution remains fairly regular (and a sign condition holds in the case without surface tension). Thus, numerical instabilities are ruled out even in a fully nonlinear regime. The analysis is based on delicate energy estimates, following a framework previously developed in the continuous case [Beale, Hou, and Lowengrub, Comm. Pure Appl. Math., 46 (1993), pp. 1269-1301]. No analyticity assumption is made for the physical solution. Our study indicates that the numerical methods must satisfy certain compatibility conditions in order to be stable. Violation of these conditions will lead to numerical instabilities. A breaking wave is calculated as an illustration.
\end{abstract}

Key words. water waves, boundary integral methods, surface tension

AMS subject classifications. Primary, 65M12; Secondary, 76B15

1. Introduction. In this paper we prove convergence of certain boundary integral methods for time-dependent, two-dimensional water waves, that is, waves on the surface of an inviscid, incompressible fluid in irrotational motion under the influence of gravity and possibly surface tension. Boundary integral methods have been used extensively for calculating the motion of fluid interfaces. A partial list of works, discussed further below, includes [And, BMO, BN, BS, CS, Do, DY, GAS, Kerr, Kral, Kra2, LC, Moor, NMP, Pull, RS, Rob, Shel, SS, Tryg, VB, WBJ, Ye]. In boundary integral methods, the interface is tracked by markers which move with the fluid. While there are many variants, a key feature is that only quantities on the surface need to be computed because of the irrotationality. For water waves, the velocity field is expressed as a singular integral, determined by the position of the interface and one more scalar quantity, which could be the velocity potential, dipole sheet strength, or vortex sheet strength. The formulation used here is closely related to that of [BMO, Bak]. Numerical instabilities have been encountered and dealt with in computations, and linear analysis at equilibrium has identified sources of instability [Rob, Do, BN], but there has been no complete error analysis of boundary integral methods. (An exception is the ill-posed case of vortex sheets for which the solutions must be assumed analytic [CL, HLK].) The rigorous proof of convergence of the particular numerical methods for water waves presented here means that numerical instabilities are ruled out even in the fully nonlinear regime, as long as the solution remains fairly regular. A filtering in high wave numbers is used in evaluating the singular integrals occurring in the time step in order to maintain the numerical stability; the methods are designed so that accuracy is preserved even with this filtering. A calculation of a breaking wave without surface tension is presented as an example of the capability of these methods (see Figures 6-12).

Error analysis for boundary integral methods is difficult, especially far away from equilibrium, because of the nonstandard nature of the equations. Our convergence proof uses energy

\footnotetext{
${ }^{*}$ Received by the editors November 11, 1993; accepted for publication (in revised form) November 7, 1994.

${ }^{\dagger}$ Mathematics Department, Box 90320, Duke University, Durham, NC 27708 (beale@math.duke.edu). The research of this author was supported by NSF grant DMS-9102782. Research at the M.S.R.I. was supported in part by NSF grant DMS-8505550.

${ }^{\ddagger}$ Department of Applied Mathematics, California Institute of Technology, Pasadena, CA 91125 (hou@ ama.caltech.edu). The research of this author was supported by a Sloan Foundation Research Fellowship, NSF grant DMS-9003202, and in part by AFOSR grant AFOSR-90-0090 and NSF grant DMS-9100383.

$\S$ Department of Mathematics, University of Minnesota, Minneapolis, MN 55455 (lowengrb@math.umn.edu) The research of this author was supported by an NSF Postdoctoral Fellowship, the University of Minnesota Army High Performance Computing Research Center, and the Minnesota Supercomputer Center.
} 
estimates in discrete Sobolev spaces with norms measuring several derivatives. In order to maintain numerical stability, balances among terms with singular integrals and derivatives have to be taken into account. Examples indicate that poorly chosen discretizations can indeed lead to numerical instabilities. Our analysis follows a framework developed in the continuous case for linearized motion perturbed about an arbitrary exact solution [BHL1]. A balance of important terms was observed in the continuous equations. However, new difficulties arise at the discrete level. The discretization must not introduce new instabilities in the high modes, and this requires that certain compatibility conditions be imposed in the way various terms in the equations are discretized. For this reason, the choice of rules for integration and differentiation, as well as the placement of the filtering, is interdependent and affects whether or not the numerical method is stable.

We now state the water wave problem in a form which leads naturally to the numerical method of interest. This formulation is very close to that of [BMO] for the more general case of an interface separating two fluids. For simplicity we assume that the fluid has infinite depth. The fluid interface is parametrized by a complex variable $z(\alpha, t)$ at the time $t$. The parameter $\alpha$ is the Lagrangian variable. Further, we denote by $\phi(\alpha, t)$ the velocity potential on the interface, and the real part and the imaginary parts of $z$ as $x$ and $y$, i.e., $z(\alpha, t)=x(\alpha, t)+i y(\alpha, t)$. To obtain a system of evolution equations, we need to express the velocity potential in the fluid domain in terms of these two variables. Following [BMO] and [BHL1], we express the complex potential by a double-layer representation. Denote by $\mu(\alpha, t)$ the dipole strength to be determined from $\phi$. We can write the complex potential $\Phi$ in the fluid domain in terms of $\mu$ as the principal value integral

$$
\Phi=\frac{1}{2 \pi i} \int \frac{1}{z-z\left(\alpha^{\prime}, t\right)} \mu\left(\alpha^{\prime}, t\right) d z\left(\alpha^{\prime}\right) .
$$

The real velocity potential in the interior is

$$
\phi=\operatorname{Re} \Phi=\int \frac{\partial}{\partial n\left(\alpha^{\prime}\right)} G\left(z-z\left(\alpha^{\prime}, t\right)\right) \mu\left(\alpha^{\prime}, t\right) d s\left(\alpha^{\prime}\right),
$$

where $G(z)=(2 \pi)^{-1} \log |z|$. The normal derivative is taken outward from the fluid domain. The nonnormalized vortex sheet strength $\gamma$ is given as the Lagrangian derivative of the dipole strength, i.e., $\gamma=\mu_{\alpha}=\partial \mu / \partial \alpha$. For simplicity, we often drop the time variable from now on, but all the quantities $z, \mu, \phi$, and $\gamma$ will be time-dependent. It follows from the Plemelj formula of complex variables, or the properties of the double-layer potential, that the value of $\phi$ on the interface is given by

$$
\phi(\alpha)=\frac{1}{2} \mu(\alpha)+\operatorname{Re} \frac{1}{2 \pi i} \int \frac{1}{z(\alpha)-z\left(\alpha^{\prime}\right)} \mu\left(\alpha^{\prime}\right) d z\left(\alpha^{\prime}\right)
$$

or

$$
\phi(\alpha)=\frac{1}{2} \mu(\alpha)+\int \frac{\partial}{\partial n\left(\alpha^{\prime}\right)} G\left(z(\alpha)-z\left(\alpha^{\prime}\right)\right) \mu\left(\alpha^{\prime}\right) d s\left(\alpha^{\prime}\right) .
$$

Differentiating both sides of the $\phi$ equation with respect to $\alpha$ and integrating by parts, we obtain

$$
\phi_{\alpha}(\alpha)=\frac{1}{2} \gamma+\operatorname{Re}\left[\frac{z_{\alpha}(\alpha)}{2 \pi i} \int \frac{1}{z(\alpha)-z\left(\alpha^{\prime}\right)} \gamma\left(\alpha^{\prime}\right) d \alpha^{\prime}\right] .
$$

The complex velocity $w=u-i v$ can be obtained by differentiating the complex potential with respect to $z$. We get

$$
w=\frac{d \Phi}{d z}=\frac{1}{2 \pi i} \int \frac{1}{z-z\left(\alpha^{\prime}\right)} \gamma\left(\alpha^{\prime}\right) d \alpha^{\prime} .
$$


Using the Plemelj formula one more time, we obtain the velocity on the interface

$$
w(\alpha)=\frac{1}{2 \pi i} \int \frac{\gamma\left(\alpha^{\prime}\right)}{z(\alpha)-z\left(\alpha^{\prime}\right)} d \alpha^{\prime}+\frac{\gamma(\alpha)}{2 z_{\alpha}(\alpha)}
$$

where we have taken the limit of $z$ approaching the free surface $z(\alpha)$ from the fluid region. Since $\alpha$ is a Lagrangian coordinate, the velocity of the interface is that of the fluid below, and we obtain an evolution equation for the interface

$$
\frac{\partial z}{\partial t}(\alpha, t)=w^{*}(\alpha, t)
$$

where the asterisk denotes the complex conjugate. For the evolution of $\phi(\alpha, t)$, we use Bernoulli's equation. If we neglect surface tension, the pressure is zero at the interface. (The case with surface tension included will be treated later.) Thus, Bernoulli's equation in the Lagrangian frame is

$$
\phi_{t}-\frac{1}{2}|w|^{2}+g y=0
$$

From now on, with $z(\alpha, t)=\alpha+s(\alpha, t)$, we assume that $s(\alpha, t)$ and $\phi(\alpha, t)$ are periodic in $\alpha$ with period $2 \pi$. This implies that the flow is at rest at infinity. The singular kernel $\frac{1}{z}$ in the velocity integrand now becomes $\frac{1}{2} \cot \left(\frac{z}{2}\right)$. To summarize, we obtain a system of time evolution equations for $z$ and $\phi$ as follows:

$$
\begin{aligned}
z_{t}^{*} & =\frac{1}{4 \pi i} \int_{-\pi}^{\pi} \gamma\left(\alpha^{\prime}\right) \cot \left(\frac{z(\alpha)-z\left(\alpha^{\prime}\right)}{2}\right) d \alpha^{\prime}+\frac{\gamma(\alpha)}{2 z_{\alpha}(\alpha)} \equiv w(\alpha, t), \\
\phi_{t} & =\frac{1}{2}\left(u^{2}+v^{2}\right)-g \cdot y, \\
\phi_{\alpha} & =\frac{\gamma}{2}+\operatorname{Re}\left[\frac{z_{\alpha}}{4 \pi i} \int_{-\pi}^{\pi} \gamma\left(\alpha^{\prime}\right) \cot \left(\frac{z(\alpha)-z\left(\alpha^{\prime}\right)}{2}\right) d \alpha^{\prime}\right] .
\end{aligned}
$$

Equations (1)-(3) completely determine the motion of the system. A unique solution is specified by giving initial conditions for the interface position $z$ and the velocity potential $\phi$. It can be shown that the integral equation for the vortex sheet strength $\gamma$ can be solved in terms of $\phi[\mathrm{BMO}]$. This is done at each time; we then use the interface equation (1) and the Bernoulli equation (2) to update $z$ and $\phi$.

In order to use the system (1)-(3) for a numerical algorithm we need to make choices for a discrete derivative operator and a quadrature rule. In addition, we use a filtering of the interface location $z$. These choices must be made in conjunction. We find it natural to use a filtering related to the Fourier symbol of the derivative operator. To describe these choices further, we recall that the discrete Fourier transform is defined by

$$
\hat{u}_{k}=\frac{1}{N} \sum_{j=-N / 2+1}^{N / 2} u\left(\alpha_{j}\right) e^{-i k \alpha_{j}}, \quad \alpha_{j}=j h, \quad h=\frac{2 \pi}{N} .
$$

The inversion formula reads

$$
u\left(\alpha_{j}\right)=\sum_{k=-N / 2+1}^{N / 2} \hat{u}_{k} e^{i k \alpha_{j}}
$$


Here we assume $N$ is even. We note that $\hat{u}_{k}$ is periodic in $k$ with period $N$. A discrete derivative operator may be expressed in the Fourier transform as

$$
\left(D_{\hat{h}} f\right)_{k}=i k \rho(k h) \hat{f}_{k}, \quad k=-\frac{N}{2}+1, \ldots, \frac{N}{2} .
$$

We will always assume $\rho(\pi)=0$; this is important in some of the arguments to follow. The choice of $\rho(x)$ depends on the derivative operator. For the second-order centered difference operator, we have

$$
\rho_{2}(k h)=\frac{\sin (k h)}{k h} \geq 0 ;
$$

for the fourth-order centered differencing,

$$
\rho_{4}(k h)=\frac{8 \sin (k h)-\sin (2 k h)}{6 k h} \geq 0 ;
$$

and for the cubic spline approximation,

$$
\rho_{c}(k h)=\left[\frac{\sin (k h)}{k h} \frac{3}{2+\cos (k h)}\right] \geq 0 .
$$

It is clear that for the $r$ th-order difference operator $\rho(k h)$ satisfies

$$
|1-\rho(k h)| \leq C(k h)^{r} .
$$

Alternatively, if $D_{h}$ is a spectral derivative, applied directly in the Fourier transform, we can choose $\rho(k h)$ to be of infinite order. In this case we will assume that $\rho$ satisfies the following properties: (i) $\rho(-x)=\rho(x)$ and $\rho(x) \geq 0$; (ii) $\rho(\cdot) \in C^{2}$ and $\rho(\pi)=0$; and (iii) $\rho(x)=1$ for $|x| \leq \lambda \pi$, where $0<\lambda<1$. Property (iii) guarantees that $D_{h}$ is spectrally accurate. We denote by $S_{h}$ the pseudospectral derivative operator without smoothing. This corresponds to the case of $\rho(k h) \equiv 1$ in the definition of $D_{h}$.

The filtering or smoothing in the interface variable will be done by multiplying by $\rho(k h)$ in the Fourier transform. When $z_{j}$ is an approximation to $z\left(\alpha_{j}\right), s_{j}=z_{j}-\alpha_{j}$ will be periodic. We define $z_{j}^{p}$ as $\alpha_{j}+s_{j}^{p}$, where

$$
\hat{s}_{k}^{p}=\rho(k h) \hat{s}_{k} .
$$

It is clear that $z^{p}$ is an $r$ th-order approximation to $z$ if $\rho$ corresponds to the $r$ th-order derivative operator. Similarly, if $D_{h}$ is a spectral derivative, we take $D_{h} z_{j}$ to mean

$$
D_{h} z_{j}=1+D_{h} s_{j}=1+D_{h}\left(z_{j}-\alpha_{j}\right) .
$$

To approximate the velocity integral, given discrete functions $z_{j} \approx z\left(\alpha_{j}\right)$ and $\gamma_{j} \approx \gamma\left(\alpha_{j}\right)$, we use the alternating trapezoidal rule, with smoothing in $z_{j}$ as above:

$$
\int_{-\pi}^{\pi} \gamma\left(\alpha^{\prime}\right) \cot \left(\frac{z\left(\alpha_{i}\right)-z\left(\alpha^{\prime}\right)}{2}\right) d \alpha^{\prime} \approx \sum_{\substack{j=-N / 2+1 \\(j-i) \text { odd }}}^{N / 2} \gamma_{j} \cot \left(\frac{z_{i}^{p}-z_{j}^{p}}{2}\right) 2 h .
$$

Now we can present our numerical algorithm for the water wave equations (1)-(3) as follows:

$$
\frac{d z_{i}^{*}}{d t}=\frac{1}{4 \pi i} \sum_{\substack{j=-N / 2+1 \\(j-i) \text { odd }}}^{N / 2} \gamma_{j} \cot \left(\frac{z_{i}^{p}-z_{j}^{p}}{2}\right) 2 h+\frac{\gamma_{i}}{2\left(D_{h} z\right)_{i}} \equiv u_{i}-i v_{i},
$$




$$
\begin{aligned}
\frac{d \phi_{i}}{d t} & =\frac{1}{2}\left(u_{i}^{2}+v_{i}^{2}\right)-g y_{i} \\
D_{h} \phi_{i} & =\frac{\gamma_{i}}{2}+\operatorname{Re}\left[\frac{D_{h} z_{i}}{4 \pi i} \sum_{\substack{j=-N / 2+1 \\
(j-i) \text { odd }}}^{N / 2} \gamma_{j} \cot \left(\frac{z_{i}^{p}-z_{j}^{p}}{2}\right) 2 h\right] .
\end{aligned}
$$

These equations can be solved once initial conditions are specified for $z_{i}$ and $\phi_{i}$ and a time discretization is chosen. The integral equation (9) must be solved for $\gamma_{i}$ at each time step. Its solvability is proved in Lemma 5 below. In practice it is solved iteratively. The version of this algorithm with surface tension will be discussed later.

Error estimates will be given in terms of the discrete $L^{2}$-norm, given by

$$
\|z\|_{l^{2}}^{2}=\sum_{j=-N / 2+1}^{N / 2}\left|z_{j}\right|^{2} h .
$$

We now state the convergence theorem for the numerical method without surface tension, followed by further discussion.

THEOREM 1. Assume that an exact solution of the water wave equations is regular enough so that $z(\cdot, t), \phi(\cdot, t) \in C^{m+2}[0,2 \pi]$ and $\gamma(\cdot, t) \in C^{m+1}[0,2 \pi]$ for $m \geq 3$, and $|z(\alpha, t)-z(\beta, t)| \geq c|\alpha-\beta|$ for $0 \leq t \leq T$ and some $c>0$. Furthermore, assume the condition

$$
\left(u_{t}, v_{t}\right) \cdot n-(0,-g) \cdot n \geq c_{0}>0
$$

holds at each point on the interface. Here $(u, v)$ is the Lagrangian velocity, $n$ is the normal vector to the interface (pointing out of the fluid region), and $c_{0}$ is some constant. Suppose the numerical solution $z(t), \phi(t), \gamma(t)$ of the initial value problem is computed using algorithm (7)-(9). Then if $D_{h}$ is an rth-order derivative approximation with $r \geq 4$, we have for $h \leq$ $h_{O}(T)$

$$
\begin{aligned}
\|z(t)-z(\cdot, t)\|_{t^{2}} & \leq C(T) h^{r}, \\
\|\phi(t)-\phi(\cdot, t)\|_{t^{2}} & \leq C(T) h^{r}, \\
\|\gamma(t)-\gamma(\cdot, t)\|_{t^{2}} & \leq C(T) h^{r-1} .
\end{aligned}
$$

If $D_{h}$ is a spectral approximation as above, we have the same convergence result with $h^{r}$ replaced by $h^{m}$ in the right-hand sides.

Condition (11) simply means that the interface is not accelerating downward, normal to itself, as rapidly as the normal acceleration of gravity. It can be viewed as a generalization of the criterion of Taylor [Tay] for horizontal interfaces to rule out Rayleigh-Taylor instabilities. It appears naturally as a sign condition in the argument below, as well as in the analysis of [BHL1]. Of course the exact solution may become singular at a later time, and the theorem asserts convergence only as long as the solution is regular. Existence results for time-dependent water waves with a finite degree of smoothness are rather limited. They began with the work of Nalimov; see [Craig] and the references cited therein.

The result proved here could be extended in several ways. In the case of finite-order derivative operators, we can improve the results by using asymptotic error expansions in the spirit of Strang [Str]. Then we can improve the convergence rate for $\gamma$ to the optimal order, i.e., $h^{r}$. Also, Strang's argument would enable us to prove convergence of the scheme corresponding to the second-order centered difference approximation. While our analysis shows that one set of choices leads to a fully convergent method, it is of course possible that 
other choices could be made which are convergent; for example, a different quadrature rule might be used for the singular integrals, as has been done in practice.

We have assumed here that the depth of the water is infinite. However, this boundary integral formulation can be modified to allow for a solid bottom below the fluid; see [BMO, $\S 4]$. If the bottom is horizontal, we need only replace the fundamental solution $G$ with the half-space Green function with a Neumann boundary condition. For a more general shape we need to include a single-layer integral over the bottom in the velocity potential, leading to an additional integral term in the velocity expression; see [BMO, Eqs. (4.1)-(4.7)]. The new integral terms over the bottom are similar to those before, but filtering of the location is not needed as in the integrals in (7) and (9) on the surface since the bottom boundary does not change. We expect that the convergence proof could be modified to include this more general case.

The Fourier filtering in the discrete integrals of (7) and (9), replacing $z$ with $z^{p}$, is introduced to balance the high wave number errors introduced by $D_{h}$; this is the reason the filtering is determined by the derivative operator. It will become apparent in the analysis that numerical stability is maintained in this way. Of course, this filtering within the increment in the time step is quite different from smoothing the entire computed solution. We do not need to apply filtering to $\gamma$; when we solve for $\gamma$ in (9), it is filtered implicitly through $D_{h}$ and $z^{p}$. To illustrate that filtering is necessary for stability, we present several numerical calculations for finiteorder derivatives without using this filtering (see $\S 6$ ). It is clear that numerical instabilities dominate the calculations in a short time. In the case of the pseudospectral derivative $(\rho=1)$, the high wave number errors would in principle not be generated. However, numerical and analytical experience indicates that the pseudospectral method without smoothing introduces aliasing errors when the physical solution is underresolved (see [GO], [HLS]). Certain Fourier smoothing or dealiasing is thus required to stabilize the method. In our case with spectral derivatives, the inclusion of smoothing in the derivative is needed for our arguments, and it makes the filtering of $z$ necessary, just as for finite-order derivatives.

We treat the case with surface tension in $\S 5$. We show that in the presence of surface tension, properly designed numerical schemes are stable and convergent even in the case where the flow is unstably stratified. The manner of discretizing the spatial derivatives in the curvature is critical for stability. As before, discretization could introduce high wave number errors which destroy the stabilizing effect of surface tension at the discrete level. This can be seen by performing classical normal mode analysis around equilibrium solutions. Such analysis was done with various schemes for vortex sheets with surface tension in [BN].

To estimate the errors in the numerical solution and prove Theorem 1, we first write equations for the difference between the computed and exact solutions. The errors separate into consistency and stability parts. The stability analysis is difficult, largely because of the discretizations of the singular integrals. Individual terms occur which are as singular as those which cause Kelvin-Helmholtz instabilities in related problems which are not well posed. The terms which are linear in the error are the most important. Our approach is to identify the leading-order linear contributions and see how the various terms balance one another. To this end, we need to study the Fourier symbols of discrete singular operators, such as the discrete Hilbert transform and its variants. In [BHL1] it was found that the linearized equations have a qualitative structure surprisingly like that for the case near equilibrium. Here a similar structure is maintained, but the arguments are more complicated because of the discretization. An important consequence is that the most singular terms cancel out to the highest order. The remaining linear terms can be shown to be bounded independent of the wave number and the discretization parameter. The nonlinear terms are relatively easy to control because of the 
smallness of the truncation error, provided the solution is fairly smooth and the method is of high-enough order of accuracy. Although the continuous case treated in [BHL1] serves as a guide, the argument here is independent of the earlier work. Similar analysis can be applied to other physical problems; these will be reported in [BHL2, BHL3, BHL4, BHL5].

Numerical methods with Lagrangian markers were attempted for vortex sheets long ago by Rosenhead [Ros]. (The vortex sheet in a single fluid is a special case in that the sheet strength is conserved, and only the interface position must be computed.) Such methods for more general fluid interfaces were apparently first proposed by Birkhoff [Bir]. The first successful boundary integral method was developed by Longuet-Higgins and Cokelet [LC], who calculated plunging breakers. Boundary integral methods for the exact, time-dependent equations have been developed and used in many other works, including Vinje and Brevig [VB], Baker, Meiron, and Orszag [BMO, Bak], Pullin [Pull], Roberts [Rob], New, McIver, and Peregrine [NMP], and Dold [Do]. Early work was reviewed by Schwartz and Fenton $[\mathrm{SF}]$ and Yeung [Ye]. A related approach for water waves is based on perturbations about equilibrium in Eulerian variables. An expansion in powers of the surface height is used to calculate Fourier modes. These works include Stiassnie and Shemer [SS], West, Brueckner, and Janda [WBJ], Dommermuth and Yue [DY], Glozman, Agnon, and Stiassnie [GAS], and Craig and Sulem [CS]. The last paper [CS] has the advantage that the expansion is evidently uniform in wave number. The Fourier method of Fenton and Rienecker [FR] is Eulerian but has some features in common with Fourier versions of the boundary integral method. Methods of boundary integral type have been used even for the ill-posed cases of fluid interface motion, including vortex sheets and Rayleigh-Taylor instabilities [Moor, And, Kral, Kra2, Kerr, Tryg, BS, Shel]. Either a regularization or filtering of high wave numbers is necessary to maintain an accurate solution. Surface tension is included in some interface calculations [Pull, RS, BN, HLS]. Related methods have also been used for viscous flow [Po].

Linear analysis has contributed to the understanding of numerical instabilities for boundary integral methods. Robert [Rob] showed how to remove a sawtooth instability. Baker and Nachbin [BN] have performed Fourier analysis near equilibrium for various schemes for a vortex sheet with surface tension, identified sources of instability, and proposed new schemes which are free of linear instabilities. Dold [Do] emphasized the role of time discretization with respect to instabilities. Hou, Lowengrub, and Shelley [HLS] introduced implicit time discretization with surface tension to avoid a severe time step constraint.

The advantage of using the alternating trapezoidal quadrature is that the approximation is spectrally accurate. This and related quadrature rules have been used by several authors in the literature. Baker [Bak] used the alternating quadrature rule for a desingularized integrand in water wave calculations. It gives a quadrature similar to (6) but with a different (and desingularized) integrand. Sidi and Israeli [SI] analyzed the spectral accuracy of a midpoint rule approximation for a periodic singular integrand. They realized that the alternating quadrature rule applied to singular integrals, as in (6), gives spectral accuracy. Shelley [Shel] used scheme (6) (with Krasny's filtering) in the context of studying the vortex sheet singularity by vortex methods. By using the spectral accuracy of the alternating trapezoidal rule, Hou, Lowengrub, and Krasny gave a simplified proof of convergence of the point vorex method for vortex sheets [HLK].

The rest of the paper is organized as follows. Sections 2 and 3 are devoted to studying the consistency and stability, respectively. We present the convergence proof in $\S 4$. In $\S 5$ we consider the case when surface tension is included. In $\S 6$ we present some numerical calculations which show that there is a class of unstable discretizations for the water wave equations (some of which have been used in the past). Comparisons in both physical and 
spectral spaces are made with our stable scheme. A calculation of a breaking wave without surface tension is presented as an example of the capability of the numerical method. Finally, we prove a few technical lemmas in the appendix.

From now on, we will use $z\left(\alpha_{j}\right), \gamma\left(\alpha_{j}\right)$, and $\phi\left(\alpha_{j}\right)$ to represent the exact solution, and $z_{j}$, $\gamma_{j}$, and $\phi_{j}$ to represent the discrete solution.

2. Consistency. We first discuss the accuracy of the quadrature in (7). By change of variables $\alpha^{\prime}=\alpha+\beta$ and using periodicity, we rewrite the velocity integral as

$$
\begin{aligned}
& \int_{-\pi}^{\pi} \gamma\left(\alpha^{\prime}\right) \cot \left(\frac{z(\alpha)-z\left(\alpha^{\prime}\right)}{2}\right) d \alpha^{\prime}=\int_{-\pi}^{\pi} \gamma(\alpha+\beta) \cot \left(\frac{z(\alpha)-z(\alpha+\beta)}{2}\right) d \beta \\
& =\int_{-\pi}^{\pi}\left[\gamma(\alpha+\beta) \cot \left(\frac{z(\alpha)-z(\alpha+\beta)}{2}\right)+\frac{\gamma(\alpha)}{z_{\alpha}(\alpha)} \cot \left(\frac{\beta}{2}\right)\right] d \beta,
\end{aligned}
$$

where we have used the fact that $\cot \left(\frac{\beta}{2}\right)$ is an odd function and the integrals are principal value integrals. Note that the last integral on the right side of (12) has a removable singularity:

$$
\lim _{\beta \rightarrow 0}\left[\gamma(\alpha+\beta) \cot \left(\frac{z(\alpha)-z(\alpha+\beta)}{2}\right)+\frac{\gamma(\alpha)}{z_{\alpha}(\alpha)} \cot \left(\frac{\beta}{2}\right)\right]=\frac{\gamma(\alpha) z_{\alpha \alpha}(\alpha)}{z_{\alpha}(\alpha)^{2}}-\frac{2 \gamma_{\alpha}(\alpha)}{z_{\alpha}(\alpha)} \text {. }
$$

We define

$$
F(\alpha, \beta) \equiv \gamma(\alpha+\beta) \cot \left(\frac{z(\alpha)-z(\alpha+\beta)}{2}\right)+\frac{\gamma(\alpha)}{z_{\alpha}(\alpha)} \cot \left(\frac{\beta}{2}\right)
$$

and

$$
J_{h}\left(\alpha_{i}\right)=\sum_{j=-N / 2+1}^{N / 2} F\left(\alpha_{i}, \beta_{j}\right) h-\int_{-\pi}^{\pi} F\left(\alpha_{i}, \beta\right) d \beta, \quad \beta_{j}=j h .
$$

Recall that $z(\cdot) \in C^{m+2}[-\pi, \pi]$ and $\gamma(\cdot) \in C^{m+1}[-\pi, \pi]$. We conclude that $F(\alpha, \cdot) \in$ $C^{m}[-\pi, \pi]$. It is well known that the trapezoidal rule approximation of a smooth periodic function gives spectral accuracy $[\mathrm{DB}]$. We obtain

$$
\left|J_{h}\left(\alpha_{i}\right)\right| \leq C h^{m}\left\|D_{\beta}^{m} F\left(\alpha_{i}, \cdot\right)\right\|_{L^{1}} .
$$

Note that

$$
\begin{aligned}
2 J_{h}\left(\alpha_{i}\right)-J_{2 h}\left(\alpha_{i}\right) & =\left[2 \sum_{j=-N / 2+1}^{N / 2} F\left(\alpha_{i}, \beta_{j}\right) h-\sum_{j=-N / 4+1}^{N / 4} F\left(\alpha_{i}, \beta_{2 j}\right) 2 h\right]-\int_{-\pi}^{\pi} F\left(\alpha_{i}, \beta\right) d \beta \\
& =\sum_{\substack{j=-N / 2+1 \\
j \text { odd }}}^{N / 2} F\left(\alpha_{i}, \beta_{j}\right) 2 h-\int_{-\pi}^{\pi} F\left(\alpha_{i}, \beta\right) d \beta,
\end{aligned}
$$

which implies

$$
\sum_{\substack{j=-N / 2+1 \\ j \text { odd }}}^{N / 2} F\left(\alpha_{i}, \beta_{j}\right) 2 h-\int_{0}^{2 \pi} F\left(\alpha_{i}, \beta\right) d \beta=O\left(h^{m}\right)
$$

Substituting (12) into (15), we obtain

$$
\begin{gathered}
\sum_{\substack{-N / 2+1 \leq j \leq N / 2 \\
j \text { odd }}} \gamma\left(\alpha_{i}+\beta_{j}\right) \cot \left(\frac{z\left(\alpha_{i}\right)-z\left(\alpha_{i}+\beta_{j}\right)}{2}\right) 2 h \\
-\int_{-\pi}^{\pi} \gamma\left(\alpha_{i}+\beta\right) \cot \left(\frac{z\left(\alpha_{i}\right)-z\left(\alpha_{i}+\beta\right)}{2}\right) d \beta=O\left(h^{m}\right) .
\end{gathered}
$$


In deriving (16), we have used the fact that $\cot \left(\frac{\beta}{2}\right)$ is an odd function of $\beta$, and $N$ is even. On the other hand, we know that if $D_{h}$ is an $r$ th-order approximation to $D_{\alpha}$, then

$$
\left|D_{h} z\left(\alpha_{i}\right)-z_{\alpha}\left(\alpha_{i}\right)\right| \leq C h^{r}\|z\|_{H^{r+2}} .
$$

If $D_{h}$ corresponds to the spectral derivative operator, then we have (see, e.g., [Tad, p. 542])

$$
\left|D_{h} z\left(\alpha_{i}\right)-z_{\alpha}\left(\alpha_{i}\right)\right| \leq C h^{m}\|z\|_{H^{m+2}} .
$$

Moreover, note that corresponding to the $r$ th-order derivative operator $D_{h}$ and $z=\alpha+s(\alpha)$

$$
\left|\hat{s}_{k}^{p}-\hat{s}_{k}\right|=\left|(\rho(k h)-1) \hat{s}_{k}\right| \leq C|k|^{r} h^{r}\left|\hat{s}_{k}\right| \text {. }
$$

We have

$$
\begin{aligned}
\left|z^{p}\left(\alpha_{i}\right)-z\left(\alpha_{i}\right)\right| & \leq \sum_{|k| \leq N / 2} C h^{r}|k|^{r}\left|\hat{s}_{k}\right| \\
& \leq C h^{r} \sum_{|k| \leq N / 2}|k|^{-2}\|z\|_{H^{r+2}} \leq C h^{r}\|z\|_{H^{r+2}} .
\end{aligned}
$$

Combining (16)-(18), we have proved the following consistency result.

CONSISTENCY LEMMA. The exact solution of the water wave equations $z(\alpha, t), \phi(\alpha, t)$ satisfies the discrete equations (7)-(10) with a truncation error of size $O\left(h^{r}\right)$ for the rth-order derivative operator ( $r=m$ for the spectral derivative).

3. Stability. To obtain stability of the method, one of the terms we need to estimate is

$$
\begin{aligned}
& {\left[\frac{1}{4 \pi i} \sum_{\substack{j=-N / 2+1 \\
(j-i) \text { odd }}}^{N / 2} \gamma_{j} \cot \left(\frac{z_{i}^{p}-z_{j}^{p}}{2}\right) 2 h+\frac{\gamma_{i}}{2 D_{h} z_{i}}\right]} \\
& -\left[\frac{1}{4 \pi i} \sum_{\substack{j=-N / 2+1 \\
(j-i) \text { odd }}}^{N / 2} \gamma\left(\alpha_{j}\right) \cot \left(\frac{z^{p}\left(\alpha_{i}\right)-z^{p}\left(\alpha_{j}\right)}{2}\right) 2 h+\frac{\gamma\left(\alpha_{i}\right)}{2 D_{h} z\left(\alpha_{i}\right)}\right]
\end{aligned}
$$

in terms of $z_{j}-z\left(\alpha_{j}\right)$ and $\gamma_{j}-\gamma\left(\alpha_{j}\right)$. For technical reasons, it is easier to work with the kernel in the infinite domain than in the periodic domain. To this end, we first extend the sum over a single period to the sum over the whole line. We note that

$$
\frac{1}{2} \cot \left(\frac{z}{2}\right)=\frac{1}{z}+\sum_{k=1}^{\infty} \frac{2 z}{z^{2}-(2 k \pi)^{2}},
$$

which converges absolutely away from $z=2 \pi k$ for any integer $k$. We have

$$
\begin{aligned}
& \frac{1}{2} \sum_{\substack{j=-N / 2+1 \\
(j-i) \text { odd }}}^{N / 2} \gamma\left(\alpha_{j}\right) \cot \left(\frac{z\left(\alpha_{i}\right)-z\left(\alpha_{j}\right)}{2}\right) 2 h \\
& =\sum_{\substack{j=-N / 2+1 \\
(j-i) \text { odd }}}^{N / 2} \frac{\gamma\left(\alpha_{j}\right) 2 h}{z\left(\alpha_{i}\right)-z\left(\alpha_{j}\right)}+\sum_{k=1}^{\infty} \sum_{\substack{j=-N / 1+1 \\
(j-i) \text { odd }}}^{N / 2} \frac{2\left(z\left(\alpha_{i}\right)-z\left(\alpha_{j}\right)\right) \gamma\left(\alpha_{j}\right)}{\left(z\left(\alpha_{i}\right)-z\left(\alpha_{j}\right)\right)^{2}-(2 k \pi)^{2}} 2 h .
\end{aligned}
$$


Recall that $z(\alpha, t)=\alpha+s(\alpha, t)$ and $s(\alpha, t)$ is $2 \pi$-periodic in $\alpha$. We obtain

$$
\begin{aligned}
& \sum_{k=1}^{M} \sum_{\substack{j=-N / 2+1 \\
(j-i) \text { odd }}}^{N / 2} \frac{2\left(z\left(\alpha_{i}\right)-z\left(\alpha_{j}\right)\right) \gamma\left(\alpha_{j}\right)}{\left(z\left(\alpha_{i}\right)-z\left(\alpha_{j}\right)\right)^{2}-(2 k \pi)^{2}} 2 h \\
& =\sum_{k=1}^{M} \sum_{\substack{j=-N / 2+1 \\
(j-i) \text { odd }}}^{N / 2}\left[\frac{\gamma\left(\alpha_{j}\right)}{z\left(\alpha_{i}\right)-z\left(\alpha_{j}\right)-2 k \pi}+\frac{\gamma\left(\alpha_{j}\right)}{z\left(\alpha_{i}\right)-z\left(\alpha_{j}\right)+2 k \pi}\right] 2 h \\
& =\sum_{k=1}^{M} \sum_{\substack{j=-N / 2+1 \\
(j-i) \text { odd }}}^{N / 2}\left[\frac{\gamma\left(\alpha_{j}\right)}{z\left(\alpha_{i}\right)-\left(\alpha_{j}+2 k \pi+s\left(\alpha_{j}\right)\right)}+\frac{\gamma\left(\alpha_{j}\right)}{z\left(\alpha_{i}\right)-\left(\alpha_{j}-2 k \pi+s\left(\alpha_{j}\right)\right)}\right] 2 h .
\end{aligned}
$$

We define

$$
\alpha_{j+k N}=\alpha_{j}+2 k \pi \text {. }
$$

Then we obtain by a change of variables that

$$
\begin{aligned}
& \sum_{k=1}^{M} \sum_{\substack{j=-N / 2+1 \\
(j=i) \text { odd } \\
\left(z\left(\alpha_{i}\right)-z\left(\alpha_{j}\right)\right)^{2}-(2 k \pi)^{2}}}^{N / 2} \frac{2\left(z\left(\alpha_{i}\right)-z\left(\alpha_{j}\right)\right) \gamma\left(\alpha_{j}\right)}{(M+1 / 2)} 2 h+\sum_{\substack{j=N / 2+1 \\
(j-i) \text { odd }}}^{N\left(\alpha_{j}\right)} \frac{\gamma\left(\alpha_{j}\right)}{z\left(\alpha_{i}\right)-z\left(\alpha_{j}\right)} 2 h+\sum_{\substack{j=-N(M+1 / 2)+1 \\
(j-i) \text { odd }}}^{-N / 2} \frac{\gamma\left(\alpha_{i}\right)-z\left(\alpha_{j}\right)}{z+1} .
\end{aligned}
$$

Combining (19) and (20), we obtain

$$
\frac{1}{2} \sum_{\substack{j=-N / 2+1 \\(j-i) \text { odd }}}^{N / 2} \gamma\left(\alpha_{j}\right) \cot \left(\frac{z\left(\alpha_{i}\right)-z\left(\alpha_{j}\right)}{2}\right) 2 h=\lim _{M \rightarrow \infty} \sum_{\substack{j=-N(M+1 / 2)+1 \\(j-i) \text { ddd }}}^{N(M+1 / 2)} \frac{\gamma\left(\alpha_{j}\right)}{z\left(\alpha_{i}\right)-z\left(\alpha_{j}\right)} 2 h .
$$

Throughout the paper, we will use the notation

$$
\sum_{(j-i) \text { odd }} \frac{\gamma\left(\alpha_{j}\right)}{z\left(\alpha_{i}\right)-z\left(\alpha_{j}\right)} 2 h \equiv \lim _{M \rightarrow \infty} \sum_{\substack{j=-N(M+1 / 2)+1 \\(j-i) \text { dd }}}^{N(M+1 / 2)} \frac{\gamma\left(\alpha_{j}\right)}{z\left(\alpha_{i}\right)-z\left(\alpha_{j}\right)} 2 h .
$$

Similarly, one can show that the corresponding extension holds for the numerical solution. We have

$$
\frac{1}{2} \sum_{\substack{j=-N / 2+1 \\(j-i) \text { odd }}}^{N / 2} \gamma_{j} \cot \left(\frac{z_{i}^{p}-z_{j}^{p}}{2}\right) 2 h=\sum_{(j-i) \text { odd }} \frac{\gamma_{j}}{z_{i}^{p}-z_{j}^{p}} 2 h \equiv \lim _{M \rightarrow \infty} \sum_{\substack{j=-N(M+1 / 2)+1 \\(j-i) \text { odd }}}^{N(M+1 / 2)} \frac{\gamma_{j}}{z_{i}^{p}-z_{j}^{p}} 2 h,
$$

where we have assumed that $s_{j}^{p}=z_{j}^{p}-\alpha_{j}$ is extended periodically outside the interval $\left(-\frac{N}{2}+1 \leq j \leq \frac{N}{2}\right)$; i.e., $s_{j \pm N}^{p}=s_{j}^{p}$. Note that $z_{j}$ is different from $z\left(\alpha_{j}\right) ; z_{j}$ stands for the numerical solution obtained from our algorithm.

We define the variations for $z, \gamma$, and $\phi$ as

$$
\begin{aligned}
& \dot{z}_{j}=z_{j}-z\left(\alpha_{j}\right), \\
& \dot{\gamma}_{j}=\gamma_{j}-\gamma\left(\alpha_{j}\right), \\
& \dot{\phi}_{j}=\phi_{j}-\phi\left(\alpha_{j}\right) .
\end{aligned}
$$

These variations define the errors between the exact and numerical solutions. 
We introduce an $m$ th-order smoothing operator $A_{-m}$ which satisfies the property

$$
\| D_{h}^{k}\left(A_{-m}\left(\dot{z}_{j}\right)\left\|_{l^{2}} \leq C\right\| \dot{z} \|_{l^{2}} \text { and }\left\|A_{-m}\left(S_{h}^{k} \dot{z}_{j}\right)\right\|_{l^{2}} \leq C\|\dot{z}\|_{l^{2}} \quad \text { for } 0 \leq k \leq m,\right.
$$

where $S_{h}$ is the spectral derivative operator without smoothing. Apparently, the second inequality also holds if we replace $S_{h}$ by $D_{h}$. In particular, when $m=0, A_{0}(\dot{z})$ denotes a bounded operator in $l^{2}$,

$$
\left\|A_{0}\left(\dot{z}_{j}\right)\right\|_{l^{2}} \leq C\|\dot{z}\|_{l^{2}}
$$

It is easy to see that

$$
h^{m} A_{0}\left(\dot{\gamma}_{j}\right)=A_{-m}\left(\dot{\gamma}_{j}\right), \quad h^{m} A_{0}\left(D_{h} \dot{z}_{j}\right)=A_{-(m-1)}\left(\dot{z}_{j}\right) .
$$

In our stability analysis, we need to study the properties of the discrete derivative operator and the discrete smoothing operator. We have the following lemmas.

LEMMA 1. Assume $f(\cdot) \in C^{3}$ and $w \in l^{2}$. Then we have

$$
D_{h}\left(f\left(\alpha_{i}\right) w_{i}\right)=f\left(\alpha_{i}\right) D_{h}\left(w_{i}\right)+w_{i}^{q} f_{\alpha}\left(\alpha_{i}\right)+h A_{0}\left(w_{i}\right),
$$

where $\hat{w}_{k}^{q}=\hat{w}_{k} q(k h)$ and $q(x)=\frac{d}{d x}(\rho(x) x), A_{0}$ is a bounded operator.

The exact form of the second term in Lemma 1 is needed for the case with surface tension.

Throughout the paper, we will frequently use the discrete "smoothing" operator of the form

$$
R_{h}\left(w_{i}\right) \equiv \sum_{(j-i) \text { odd }} f\left(\alpha_{i}, \alpha_{j}\right) w_{j} 2 h
$$

for some function $f\left(\alpha, \alpha^{\prime}\right)$ which is smooth in both $\alpha$ and $\alpha^{\prime} ; w$ is a generic periodic function. It is clear that the continuous analogue of $R_{h}$ is a smoothing operator, i.e., $R(w)=A_{-m}(w)$ if $f \in C^{m}$. However, at the discrete level, $R_{h}$ does not necessarily give a smoothing property due to aliasing errors. For example, if we let

$$
f\left(\alpha, \alpha^{\prime}\right)=\frac{1}{\pi} \frac{g(\alpha)-g\left(\alpha^{\prime}\right)}{\alpha-\alpha^{\prime}}, \quad f(\alpha, \alpha)=g^{\prime}(\alpha), \quad \text { with } g(\alpha)=e^{2 i \alpha},
$$

and $w_{i}=e^{i \alpha_{i}(N / 2-1)}$, then we can show (using Lemma 3 below) that

$$
R_{h}\left(w_{i}\right)=-2 i g\left(\alpha_{i}\right) w_{i}
$$

which is as singular as the $w$ that we started with. This shows that the discrete operator does not necessarily produce any smoothing effect on high-frequency components due to aliasing errors. However, with smoothing on the high-frequency components of $w$, we can prove the following result.

LEMMA 2. Assume $f(\alpha, \beta)$ is a smooth function in $\alpha$ and $\beta$, and $f(\cdot, \cdot) \in C^{m}$ with $m>2$. Then with $R_{h}$ defined by $(24)$ we have $R_{h}\left(w^{p}\right)=A_{-1}(w)$, i.e.,

$$
\sum_{(j-i) \text { odd }} f\left(\alpha_{i}, \alpha_{j}\right) w_{j}^{p} 2 h=A_{-1}(w) .
$$

If $\rho(x)$ satisfies, in addition, that $\rho^{\prime}( \pm \pi)=0$ and $f(\cdot, \cdot) \in C^{m}$ with $m>3$, then we have $R_{h}\left(w^{p}\right)=A_{-2}(w)$, i.e.,

$$
\sum_{(j-i) \text { odd }} f\left(\alpha_{i}, \alpha_{j}\right) w_{j}^{p} 2 h=A_{-2}(w)
$$


We will defer the proofs of these two lemmas to the appendix.

We remark that, in particular, Lemma 2 means that $R_{h}\left(S_{h} \dot{z}^{p}\right)=A_{0}(\dot{z})$, and, in the second case, $R_{h}\left(S_{h}^{2} \dot{z}^{p}\right)=A_{0}(\dot{z})$. This property will be used later in our analysis. We define

$$
E_{i} \equiv \frac{1}{2 \pi i} \sum_{(j-i) \text { odd }} \frac{\gamma_{j}}{z_{i}^{p}-z_{j}^{p}} 2 h, \quad \zeta_{i} \equiv \frac{\gamma_{i}}{D_{h} z_{i}},
$$

and

$$
Q_{i} \equiv \frac{D_{h} z_{i}}{2 \pi i} \sum_{(j-i) \text { odd }} \frac{\gamma_{j}}{z_{i}^{p}-z_{j}^{p}} 2 h .
$$

Similarly, we define $E\left(\alpha_{i}\right), \zeta\left(\alpha_{i}\right)$, and $Q\left(\alpha_{i}\right)$ with $z_{i}$ replaced by $z\left(\alpha_{i}\right)$ and $\gamma_{i}$ by $\gamma\left(\alpha_{i}\right)$. Now we need to estimate the variances of these three variables:

$$
\dot{E}_{i}=E_{i}-E\left(\alpha_{i}\right), \quad \dot{\zeta}_{i}=\zeta_{i}-\zeta\left(\alpha_{i}\right), \quad \dot{Q}_{i}=Q_{i}-Q\left(\alpha_{i}\right) .
$$

These variations define the errors between the exact quantity and its numerical counterpart.

We first estimate $\dot{E}$. We decompose $\dot{E}$ into its linear part and the nonlinear part

$$
\dot{E}_{i}=\dot{E}_{i}^{L}+\dot{E}_{i}^{N L} \text {. }
$$

Direct calculations show that the linear part $\dot{E}_{i}^{L}$ is given by

$$
\dot{E}_{i}^{L} \equiv \frac{1}{2 \pi i} \sum_{(j-i) \text { odd }} \frac{\dot{\gamma}_{j}}{z\left(\alpha_{i}\right)^{p}-z\left(\alpha_{j}\right)^{p}} 2 h-\frac{1}{2 \pi i} \sum_{(j-i) \text { odd }} \frac{\gamma\left(\alpha_{j}\right)\left(\dot{z}_{i}^{p}-\dot{z}_{j}^{p}\right)}{\left(z\left(\alpha_{i}\right)^{p}-z\left(\alpha_{j}\right)^{p}\right)^{2}} 2 h,
$$

and the nonlinear part $E_{i}^{N L}$ is given by

$$
\begin{aligned}
\dot{E}_{i}^{N L} \equiv & \frac{1}{2 \pi i} \sum_{(j-i) \text { odd }}\left[\frac{\gamma_{j}\left(\dot{z}_{i}^{p}-\dot{z}_{j}^{p}\right)^{2}}{\left(z\left(\alpha_{i}\right)^{p}-z\left(\alpha_{j}\right)^{p}\right)^{2}\left(z\left(\alpha_{i}\right)^{p}-z\left(\alpha_{j}\right)^{p}+\dot{z}_{i}^{p}-\dot{z}_{j}^{p}\right)} 2 h\right] \\
& -\frac{1}{2 \pi i} \sum_{(j-i) \text { odd }}\left[\frac{\dot{\gamma}_{j}\left(\dot{z}_{i}^{p}-\dot{z}_{j}^{p}\right)}{\left(z\left(\alpha_{i}\right)^{p}-z\left(\alpha_{j}\right)^{p}\right)\left(z\left(\alpha_{i}\right)^{p}-z\left(\alpha_{j}\right)^{p}+\dot{z}_{i}^{p}-\dot{z}_{j}^{p}\right)} 2 h\right] .
\end{aligned}
$$

To estimate the linear part of $E_{i}$, we need to introduce a discrete Hilbert transform:

$$
H_{h} f_{i}=\frac{1}{\pi} \lim _{M \rightarrow \infty} \sum_{\substack{j=-N(M+1 / 2)+1 \\(j-i) \text { odd }}}^{N(M+1 / 2)} \frac{f_{j}}{\alpha_{i}-\alpha_{j}} 2 h \equiv \frac{1}{\pi} \sum_{(j-i) \text { odd }} \frac{f_{j}}{\alpha_{i}-\alpha_{j}} 2 h,
$$

where $M>0$ is an integer. In the appendix, we will prove that this discrete Hilbert transform shares many properties of the continuous Hilbert transform. These are summarized in the following lemma.

LEMMA 3. Assume that $f$ satisfies $\hat{f}_{0}=\hat{f}_{N / 2}=0$. The discrete Hilbert transform defined by (30) satisfies the following properties:

$$
\begin{aligned}
H_{\hat{h}} f_{k} & =-i \operatorname{sgn}(k) \hat{f}_{k}, \\
H_{h}\left(H_{h} f_{i}\right) & =-f_{i}, \\
H_{h}\left(S_{h} f_{i}\right) & =\frac{1}{\pi} \sum_{(j-i) \text { odd }} \frac{f_{i}-f_{j}}{\left(\alpha_{i}-\alpha_{j}\right)^{2}} 2 h, \\
S_{h}\left(H_{h} f_{i}\right) & =H_{h}\left(S_{h} f_{i}\right),
\end{aligned}
$$

where $S_{h}$ is the pseudospectral derivative operator without smoothing. 
Using Lemmas 2 and 3, we can prove the following.

LEMMA 4. Assume $z(\cdot, t) \in C^{m+2}(m \geq 3), \gamma(\cdot, t) \in C^{m+1}$, and $z_{\alpha} \neq 0$. Then

$$
\begin{aligned}
\dot{E}_{i}^{L} & =\frac{1}{2 \pi i} \sum_{(j-i) \text { odd }} \frac{\dot{\gamma}_{j}}{z\left(\alpha_{i}\right)^{p}-z\left(\alpha_{j}\right)^{p}} 2 h-\frac{\gamma\left(\alpha_{i}\right)}{2 i z_{\alpha}\left(a_{i}\right)^{2}} H_{h} D_{h}\left(\dot{z}_{i}\right)+A_{0}\left(\dot{z}_{i}\right) \\
& =\frac{1}{2 i z_{\alpha}\left(\alpha_{i}\right)} H_{h}\left(\dot{\gamma}_{i}\right)-\frac{\gamma\left(\alpha_{i}\right)}{2 i z_{\alpha}\left(\alpha_{i}\right)^{2}} H_{h} D_{h}\left(\dot{z}_{i}\right)+A_{0}\left(\dot{z}_{i}\right)+R_{h}(\dot{\gamma})+A_{-1}(\dot{\gamma}),
\end{aligned}
$$

where $R_{h}$ is an operator of the type (24) with a smooth kernel.

We defer the proof to the appendix.

Remark. Note that since we do not introduce smoothing in $\dot{\gamma}$ explicitly, we do not have $R_{h}(\dot{\gamma})=A_{-1}(\dot{\gamma})$ directly. As we will see in $(60), \dot{\gamma}$ is related to $D_{h} \dot{\phi}$ and $D_{h} \dot{z}$. Since $D_{h} \dot{z}=\left(S_{h} \dot{z}\right)^{p}$, Lemma 2 implies that $R_{h}\left(D_{h} \dot{z}\right)=A_{-1}\left(S_{h} \dot{z}\right)=A_{0}(\dot{z})$. Similarly we have $R_{h}\left(D_{h} \dot{\phi}\right)=A_{0}(\dot{\phi})$. This is sufficient to close our energy estimates. And this is why we do not need to filter $\dot{\gamma}$ in our numerical method.

We now estimate $\dot{\zeta}$. Again, we decompose $\dot{\zeta}$ into its linear part and the nonlinear part, $\dot{\zeta}_{j}=\dot{\zeta}_{j}^{L}+\dot{\zeta}_{j}^{N L}$, where the linear part $\dot{\zeta}_{j}^{L}$ is given by

$$
\dot{\zeta}_{j}^{L}=\frac{D_{h} z\left(\alpha_{j}\right) \dot{\gamma}_{j}-\gamma\left(\alpha_{j}\right) D_{h} \dot{z}_{j}}{D_{h} z\left(\alpha_{j}\right)^{2}}=\frac{z_{\alpha}\left(\alpha_{j}\right) \dot{\gamma}_{j}-\gamma\left(\alpha_{j}\right) D_{h} \dot{z}_{j}}{z_{\alpha}\left(\alpha_{j}\right)^{2}}+A_{-r}\left(\dot{\gamma}_{j}\right)+A_{-1}\left(\dot{z}_{j}\right),
$$

and the nonlinear part $\dot{\zeta}_{j}^{N L}$ is given by

$$
\dot{\zeta}_{j}^{N L}=-\frac{\left(D_{h} z\left(\alpha_{j}\right) \dot{\gamma}_{j}-\gamma\left(\alpha_{j}\right) D_{h} \dot{z}_{j}\right) D_{h} \dot{z}_{j}}{D_{h} z\left(\alpha_{j}\right)^{2}\left(D_{h} z\left(\alpha_{j}\right)+D_{h} \dot{z}_{j}\right)} .
$$

Note from Lemma 2 that the commutator of $\left[H_{h}, f\right]$ is a smoothing operator:

$$
\begin{aligned}
{\left[H_{h}, f\right] \dot{z}_{i}^{p} } & \equiv H_{h}\left(f\left(\alpha_{i}\right) \dot{z}_{i}^{p}\right)-f\left(\alpha_{i}\right) H_{h}\left(\dot{z}_{i}^{p}\right) \\
& =\sum_{(j-i) \text { odd }} \frac{f\left(\alpha_{i}\right)-f\left(\alpha_{j}\right)}{\alpha_{i}-\alpha_{j}} \dot{z}_{j}^{p} 2 h=A_{-1}\left(\dot{z}_{i}\right),
\end{aligned}
$$

since $g(\alpha, \beta)=\frac{f(\alpha)-f(\beta)}{\alpha-\beta}$ is a smooth function in both $\alpha$ and $\beta$. Here we define $g(\alpha, \alpha)=$ $f^{\prime}(\alpha)$. Thus, we obtain from Lemma 1 and the above estimate on the commutator operator that

$$
\dot{E}_{i}^{L}+\frac{1}{2} \dot{\zeta}_{i}^{L}=\frac{1}{2 z_{\alpha}}\left[\left(I-i H_{h}\right)\left(\dot{\gamma}_{i}-\frac{\gamma}{z_{\alpha}} D_{h} \dot{z}_{i}\right)\right]+A_{0}\left(\dot{z}_{i}\right)+R_{h}\left(\dot{\gamma}_{i}\right)+A_{-1}\left(\dot{\gamma}_{i}\right),
$$

where we have used $A_{-1}\left(D_{h} \dot{z}\right)=A_{0}(\dot{z})$.

Remark. We remark that if we had not used the filtering $z^{p}$ in approximating the velocity integral, then the term $H_{h} D_{h}(\dot{z})$ in Lemma 4 would have become $H_{h} S_{h}(\dot{z})$. In that case, the leading-order terms of (39) would become

$$
\frac{1}{2 z_{\alpha}}\left(\dot{\gamma}_{i}-\frac{\gamma}{z_{\alpha}} D_{h} \dot{z}_{i}\right)-\left(\frac{i}{2 z_{\alpha}} H_{h}(\dot{\gamma})-\frac{i \gamma}{2 z_{\alpha}^{2}} H_{h} S_{h}(\dot{z})\right) .
$$

The high-mode errors between $H_{h} D_{h}$ and $H_{h} S_{h}$ would not allow us to combine $\dot{E}^{L}$ with $\dot{\zeta}^{L}$ into one term, as in (39). It can be seen that this upsets the high-mode balances and introduces numerical instability. This explains why we need to introduce the additional filtering in $z$ to 
compensate for the high-frequency errors introduced by $D_{h}$. Another benefit we have by using the Fourier filtering is to smooth out the aliasing errors in the commutator $\left[H_{h}, f\right]$ (see (38)).

We define

$$
T^{*}=\sup \left\{t \mid t \leq T,\|\dot{z}\|_{l^{2}},\|\dot{\phi}\|_{l^{2}} \leq h^{5 / 2},\|\dot{\gamma}\|_{l^{2}} \leq h^{3 / 2}\right\}
$$

Note that

$$
h\left|\dot{z}_{i}\right|^{2} \leq h \sum_{i=1}^{N}\left|\dot{z}_{i}\right|^{2}=\|\dot{z}\|_{l^{2}}^{2}
$$

We conclude that

$$
\|\dot{z}\|_{\infty} \leq h^{-1 / 2}\|\dot{z}\|_{l^{2}} \leq h^{2} \text { for } t \leq T^{*}
$$

and

$$
\|\dot{\gamma}\|_{\infty} \leq h^{-1 / 2}\|\dot{\gamma}\|_{l^{2}} \leq h \quad \text { for } t \leq T^{*}
$$

Note that

$$
\left\|D_{h} \dot{z}\right\|_{l^{2}}^{2}=\sum_{k=1}^{N}(k \rho(k h))^{2}\left|\hat{\dot{z}}_{k}\right|^{2} \leq N^{2} \sum_{k=1}^{N}\left|\hat{\dot{z}}_{k}\right|^{2}=\left(\frac{2 \pi}{h}\right)^{2}\|\dot{z}\|_{l^{2}}^{2} .
$$

Therefore, we obtain

$$
\left\|D_{h} \dot{z}\right\|_{\infty} \leq h^{-1 / 2}\left\|D_{h} \dot{z}\right\|_{l^{2}} \leq 2 \pi h^{-3 / 2}\|\dot{z}\|_{l^{2}}
$$

As a result,

$$
\left\|D_{h} \dot{z}\right\|_{\infty} \leq 2 \pi h \text { for } t \leq T^{*} .
$$

Now, since $D_{h} z=z_{\alpha}+O\left(h^{r}\right), z_{\alpha} \neq 0$ for $t \leq T^{*}$, we have from (37) that

$$
\left\|\dot{\zeta}^{N L}\right\|_{l^{2}} \leq C\left\|D_{h} \dot{z}\right\|_{l^{2}}\left(\|\dot{\gamma}\|_{\infty}+\left\|D_{h} \dot{z}\right\|_{\infty}\right) \leq C h\left\|D_{h} \dot{z}\right\|_{l^{2}} .
$$

In view of (42), we conclude that

$$
\left\|\dot{\zeta}^{N L}\right\|_{l^{2}} \leq C\|\dot{z}\|_{l^{2}}
$$

which implies that

$$
\dot{\zeta}^{N L}=A_{0}(\dot{z}) \quad \text { for } t \leq T^{*} .
$$

We are now left to estimate the nonlinear term $\dot{E}_{j}^{N L}$ defined in (29). It is sufficient to illustrate the idea for one of the two terms. The other can be treated similarly. We take the second term on the right-hand side of (29) as an example. By assumption we know that

$$
\left|z\left(\alpha_{i}\right)-z\left(\alpha_{j}\right)\right| \geq c\left|\alpha_{i}-\alpha_{j}\right| \geq c h \text { for } j-i \text { odd. }
$$

Therefore, for $h$ small and $t \leq T^{*}$, we have, by using (41),

$$
\left|z\left(\alpha_{i}\right)-z\left(\alpha_{j}\right)+\dot{z}_{i}-\dot{z}_{j}\right| \geq c\left|\alpha_{i}-\alpha_{j}\right|-2 h^{2} \geq \frac{c}{2}\left|\alpha_{i}-\alpha_{j}\right| .
$$


Thus, for $t \leq T^{*}$,

$$
\begin{aligned}
& \left\|\frac{1}{2 \pi i} \sum_{(j-i) \text { odd }} \frac{\dot{\gamma}_{j}\left(\dot{z}_{i}^{p}-\dot{z}_{j}^{p}\right) 2 h}{\left(z\left(\alpha_{i}\right)-z\left(\alpha_{j}\right)+\dot{z}_{i}-\dot{z}_{j}\right)\left(z\left(\alpha_{i}\right)-z\left(\alpha_{j}\right)\right)}\right\|_{l^{2}} \\
\leq & \|\dot{\gamma}\|_{\infty}\|\dot{z}\|_{l^{2}} \max _{i} \sum_{(j-i) \text { odd }} \frac{c h}{\left.\mid \alpha_{i}-\alpha_{j}\right)^{2}} \leq \frac{c}{h}\|\dot{\gamma}\|_{\infty}\|\dot{z}\|_{l^{2}} \leq c\|\dot{z}\|_{l^{2}},
\end{aligned}
$$

where we have used Young's inequality in the estimate that involves $\dot{z}_{j}$ in the numerator. The above argument still applies when we replace $z$ by $z^{p}$ since $z^{p}=z+O\left(h^{r}\right)$ with $r \geq 2$. This shows that

$$
\frac{1}{2 \pi i} \sum_{(j-i) \text { odd }} \frac{\dot{\gamma}_{j}\left(\dot{z}_{i}^{p}-\dot{z}_{j}^{p}\right) 2 h}{\left(z\left(\alpha_{i}\right)^{p}-z\left(\alpha_{j}\right)^{p}+\dot{z}_{i}^{p}-\dot{z}_{j}^{p}\right)\left(z\left(\alpha_{i}\right)^{p}-z\left(\alpha_{j}\right)^{p}\right)}=A_{0}\left(\dot{z}_{i}\right) .
$$

Similarly, we can show that the first term in (29) is $A_{0}\left(\dot{z}_{i}\right)$. This proves

$$
\dot{E}_{i}^{N L}=A_{0}(\dot{z}) \quad \text { for } t \leq T^{*} .
$$

Putting (39), (44), and (48) together, we obtain

$$
\dot{E}_{j}+\frac{1}{2} \dot{\zeta}_{j}=\frac{1}{2 z_{\alpha}\left(\alpha_{j}\right)}\left(I-i H_{h}\right)\left(\dot{\gamma}_{j}-\frac{\gamma\left(\alpha_{j}\right)}{z_{\alpha}\left(\alpha_{j}\right)} D_{h} \dot{z}_{j}\right)+A_{0}\left(\dot{z}_{j}\right)+A_{-1}\left(\dot{\gamma}_{j}\right)+R_{h}\left(\dot{\gamma}_{j}\right)
$$

This completes the estimate for $E_{j}+\zeta_{j} / 2$.

We now turn to estimate $\dot{Q}_{j}$ defined below (26). It is easy to see that

$$
\begin{aligned}
\dot{Q}_{i} & =\left(D_{h} \dot{z}_{i}\right) E_{i}+D_{h} z\left(\alpha_{i}\right) \dot{E}_{i} \\
& =\left(D_{h} \dot{z}_{i}\right)\left(E\left(\alpha_{i}\right)+\dot{E}_{i}\right)+D_{h} z\left(\alpha_{i}\right) \dot{E}_{i},
\end{aligned}
$$

where $E_{i}$ is defined as before. It is easy to show that $\|\dot{E}\|_{\infty} \leq c h$ for $t \leq T^{*}$. On the other hand, from the consistency argument, we have

$$
E\left(\alpha_{i}\right)=\frac{1}{2 \pi i} \int \frac{\gamma\left(\alpha^{\prime}\right)}{z\left(\alpha_{i}\right)-z\left(\alpha^{\prime}\right)} d \alpha^{\prime}+O\left(h^{r}\right) \equiv w_{0}\left(\alpha_{i}\right)+O\left(h^{r}\right),
$$

where

$$
w_{0}\left(\alpha_{i}\right)=\frac{1}{2 \pi i} \int \frac{\gamma\left(\alpha^{\prime}\right)}{z\left(\alpha_{i}\right)-z\left(\alpha^{\prime}\right)} d \alpha^{\prime}
$$

Moreover, we have

$$
D_{h} z\left(\alpha_{i}\right)=z_{\alpha}\left(\alpha_{i}\right)+O\left(h^{r}\right) .
$$

Using the first estimate for $\dot{E}^{L}$ in Lemma 4 and the previous estimate for $\dot{E}_{i}^{N L}$, we obtain that for $t \leq T^{*}$

$$
\begin{aligned}
\dot{Q}_{i}= & D_{h} \dot{z}_{i} w_{0}\left(\alpha_{i}\right)+\frac{D_{h} z\left(\alpha_{i}\right)}{2 \pi i} \sum_{(j-i) \text { odd }} \frac{\dot{\gamma}_{j}}{z\left(\alpha_{i}\right)^{p}-z\left(\alpha_{j}\right)^{p}} 2 h \\
& -\frac{\gamma\left(\alpha_{i}\right)}{2 i z_{\alpha}\left(\alpha_{i}\right)} H_{h} D_{h}\left(\dot{z}_{i}\right)+A_{0}\left(\dot{z}_{i}\right) .
\end{aligned}
$$

Equations (49) and (50) are our main stability estimates in this section. 
4. Energy estimates and convergence. We are ready to combine our consistency and stability estimates to obtain convergence of the method. It follows from the consistency lemma and the definition of $\dot{E}, \dot{\zeta}$, and $\dot{Q}$ that

$$
\begin{gathered}
\frac{d}{d t} \dot{z}_{i}^{*}=\dot{E}_{i}+\frac{1}{2} \dot{\zeta}_{i}+O\left(h^{r}\right), \\
D_{h} \dot{\phi}_{i}=\frac{\dot{\gamma}_{i}}{2}+\operatorname{Re}\left\{\dot{Q}_{i}\right\}+O\left(h^{r}\right) .
\end{gathered}
$$

Substituting estimates (49) and (50) into the above equations, we get

$$
\begin{aligned}
\frac{d \dot{z}_{i}^{*}}{d t}= & \frac{1}{2 z_{\alpha}\left(\alpha_{i}\right)}\left(I-i H_{h}\right)\left[\dot{\gamma}_{i}-\frac{\gamma\left(\alpha_{i}\right)}{z_{\alpha}\left(\alpha_{i}\right)} D_{h} \dot{z}_{i}\right]+A_{0}(\dot{z}) \\
& +A_{-1}\left(\dot{\gamma}_{i}\right)+R_{h}\left(\dot{\gamma}_{j}\right)+O\left(h^{r}\right), \\
D_{h} \dot{\phi}_{i}= & \frac{\dot{\gamma}_{i}}{2}+\operatorname{Re}\left[D_{h} \dot{z}_{i} w_{0}\left(\alpha_{i}\right)-\frac{\gamma\left(\alpha_{i}\right)}{2 i z_{\alpha}\left(\alpha_{i}\right)} H_{h} D_{h}(\dot{z})\right] \\
& +\operatorname{Re}\left[\frac{D_{h} z\left(\alpha_{i}\right)}{2 \pi i} \sum_{(j-i) \text { odd }} \frac{\dot{\gamma}_{j}}{z\left(\alpha_{i}\right)^{p}-z\left(\alpha_{j}\right)^{p}} 2 h\right]+A_{0}\left(\dot{z}_{i}\right)+O\left(h^{r}\right) .
\end{aligned}
$$

Furthermore, we can write (54) as

$$
\begin{aligned}
D_{h} \dot{\phi}_{i}= & \frac{1}{2} \dot{\gamma}_{i}+\left(K_{h} \dot{\gamma}_{i}\right)+\operatorname{Re}\left\{w_{0}\left(\alpha_{i}\right) D_{h} \dot{z}_{i}\right\}-\operatorname{Im}\left[\frac{\gamma\left(\alpha_{i}\right)}{2 z_{\alpha}\left(\alpha_{i}\right)} H_{h} D_{h}\left(\dot{z}_{i}\right)\right] \\
& +A_{0}\left(\dot{z}_{i}\right)+O\left(h^{r}\right),
\end{aligned}
$$

where

$$
K_{h} \dot{\gamma}_{i}=\operatorname{Re}\left[\frac{D_{h} z\left(\alpha_{i}\right)}{2 \pi i} \sum_{(j-i) \text { odd }} \frac{\dot{\gamma}_{j}}{z\left(\alpha_{i}\right)^{p}-z\left(\alpha_{j}\right)^{p}} 2 h\right]
$$

We remark that $\dot{\phi}$ and $\dot{\gamma}$ are not independent. Actually, one can solve for $\dot{\gamma}$ in terms of $\dot{\phi}$ and $\dot{z}$ in (54). In the rest of the paper, we will use $\dot{z}$ and $\dot{\phi}$ as our unknowns. So we need to express $\dot{\gamma}$ in terms of $\dot{z}$ and $\dot{\phi}$. From (54) we have

$$
\left(\frac{1}{2} I+K_{h}\right) \dot{\gamma}_{i}=D_{h} \dot{\phi}_{i}-\operatorname{Re}\left[w_{0}\left(\alpha_{i}\right) D_{h} \dot{z}_{i}-\frac{\gamma\left(\alpha_{i}\right)}{2 i z_{\alpha}\left(\alpha_{i}\right)} H_{h} D_{h}\left(\dot{z}_{i}\right)\right]+A_{0}\left(\dot{z}_{i}\right)+O\left(h^{r}\right) .
$$

Apparently, if $\left(\frac{1}{2} I+K_{h}\right)^{-1}$ is bounded, then we can bound $\dot{\gamma}$ in terms of $D_{h} \dot{\phi}, D_{h} \dot{z}$ and $O\left(h^{r}\right)$.

LEMMA 5. Assume $z(\cdot, t) \in C^{3}$ and $z_{\alpha} \neq 0$. Then there exists a constant $h_{0}>0$ and $C>0$, such that for all $h$ with $0<h \leq h_{0}$,

$$
\left\|\left(\frac{1}{2} I+K_{h}\right)^{-1}\right\|_{l^{2}} \leq C .
$$

The proof of Lemma 5 will be deferred to the appendix.

It follows from (55) and (56) that

$$
\dot{\gamma}_{i}=-2 K_{h} \dot{\gamma}+2 D_{h} \dot{\phi}_{i}-2 \operatorname{Re}\left(w_{0}\left(\alpha_{i}\right) D_{h} \dot{z}_{i}\right)+\operatorname{Im}\left[\frac{\gamma\left(\alpha_{i}\right)}{z_{\alpha}\left(\alpha_{i}\right)} H_{h} D_{h}\left(\dot{z}_{i}\right)\right]+A_{0}\left(\dot{z}_{i}\right)+O\left(h^{r}\right) .
$$


We will show that $K_{h} \dot{\gamma}$ is a lower-order term. To see this, we first rewrite (57) as

$$
\left(\frac{1}{2} I+K_{h}\right) \dot{\gamma}=D_{h} \dot{G}+A_{0}(\dot{z})+O\left(h^{r}\right)
$$

where

$$
\dot{G} \equiv \dot{\phi}-\operatorname{Re}\left[w_{0}\left(\alpha_{i}\right) \dot{z}_{i}-\frac{\gamma\left(\alpha_{i}\right)}{2 i z_{\alpha}\left(\alpha_{i}\right)} H_{h}\left(\dot{z}_{i}\right)\right]
$$

and we have used Lemma 1. Using (59) we have

$$
\begin{aligned}
K_{h} \dot{\gamma} & =K_{h}\left(\frac{1}{2} I+K_{h}\right)^{-1}\left(D_{h} \dot{G}+A_{0}(\dot{z})+O\left(h^{r}\right)\right) \\
& =\left(\frac{1}{2} I+K_{h}\right)^{-1} K_{h}\left(D_{h} \dot{G}+A_{0}(\dot{z})+O\left(h^{r}\right)\right),
\end{aligned}
$$

where we have used

$$
K_{h}\left(\frac{1}{2} I+K_{h}\right)^{-1}=\left(\frac{1}{2} I+K_{h}\right)^{-1} K_{h}
$$

which can be verified easily. Furthermore, we note that

$$
K_{h} \dot{\gamma}=\operatorname{Re}\left[\frac{1}{2 \pi i} \sum_{(j-i) \text { odd }} \frac{\dot{\gamma}_{j}}{\alpha_{i}-\alpha_{j}} 2 h\right]+\sum_{(j-i) \text { odd }} g\left(\alpha_{i}, \alpha_{j}\right) \dot{\gamma}_{j} 2 h=\sum_{(j-i) \text { odd }} g\left(\alpha_{i}, \alpha_{j}\right) \dot{\gamma}_{j} 2 h
$$

for some smooth function $g$. Thus, $K_{h}(\dot{\gamma})$ is an operator of the type (24). Therefore, we obtain by using Lemma 2 that

$$
K_{h}\left(D_{h} \dot{G}\right)=K_{h}\left(\left(S_{h} \dot{G}\right)^{p}\right)=A_{-1}\left(S_{h} \dot{G}\right)=A_{0}(\dot{G}) .
$$

Since $\left(\frac{1}{2} I+K_{h}\right)^{-1}$ is a bounded operator, we conclude that

$$
K_{h} \dot{\gamma}=A_{0}(\dot{z})+A_{0}(\dot{\phi})+O\left(h^{r}\right) .
$$

As a result, we get

$$
\begin{aligned}
\dot{\gamma}_{i}= & 2 D_{h} \dot{\phi}_{i}-2 \operatorname{Re}\left(w_{0}\left(\alpha_{i}\right) D_{h} \dot{z}_{i}\right)+D_{h} H_{h} \operatorname{Im}\left[\frac{\gamma\left(\alpha_{i}\right)}{z_{\alpha}\left(\alpha_{i}\right)} \dot{z}_{i}\right] \\
& +A_{0}\left(\dot{z}_{i}\right)+A_{0}\left(\dot{\phi}_{i}\right)+O\left(h^{r}\right),
\end{aligned}
$$

where we have used Lemma 1 and the estimate (38) on the commutator $\left[H_{h}, f\right]$. In (53) we have terms of the form $R_{h}(\dot{\gamma})$ and $A_{-1}(\dot{\gamma})$. These can now be rewritten as error terms in $\dot{z}$ and $\dot{\phi}$. For the first term in (60), for example, we have $R_{h}\left(D_{h} \dot{\phi}\right)=R_{h}\left(S_{h} \dot{\phi}^{p}\right)=A_{0}(\dot{\phi})$, using Lemma 2. Treating other terms in (60) similarly, and using Lemma 1 in the second term, we obtain

$$
R_{h}(\dot{\gamma})=A_{0}(\dot{z})+A_{0}(\dot{\phi})+O\left(h^{r}\right)
$$

and

$$
A_{-1}(\dot{\gamma})=A_{0}(\dot{z})+A_{0}(\dot{\phi})+O\left(h^{r}\right)
$$


Note that from Lemma 3 ,

$$
\left(I-i H_{h}\right)\left(I+i H_{h}\right) D_{h}=\left(I+H_{h}^{2}\right) D_{h}=0,
$$

since both the zeroth mode and the $\frac{N}{2}$ th mode of $D_{h}$ vanish. So we get

$$
\left(I-i H_{h}\right) H_{h} D_{h}=\left(I-i H_{h}\right)\left(i D_{h}\right) .
$$

Now applying $\left(I-i H_{h}\right)$ to both sides of (60), we get

$$
\begin{aligned}
\left(I-i H_{h}\right) \dot{\gamma}_{i}= & \left(I-i H_{h}\right)\left(2 D_{h} \dot{\phi}_{i}-2 \operatorname{Re}\left(w_{0}\left(\alpha_{i}\right) D_{h} \dot{z}_{i}\right)\right) \\
& +\left(I-i H_{h}\right)\left[H_{h} D_{h} \operatorname{Im}\left(\frac{\gamma\left(\alpha_{i}\right)}{z_{\alpha}\left(\alpha_{i}\right)} \dot{z}_{i}\right)\right]+A_{0}(\dot{z})+A_{0}(\dot{\phi})+O\left(h^{r}\right) .
\end{aligned}
$$

It follows from (63) that

$$
\left(I-i H_{h}\right)\left[H_{h} D_{h} \operatorname{Im}\left(\frac{\gamma\left(\alpha_{i}\right)}{z_{\alpha}\left(\alpha_{i}\right)} \dot{z}_{i}\right)\right]=\left(I-i H_{h}\right)\left[i D_{h} \operatorname{Im}\left(\frac{\gamma\left(\alpha_{i}\right)}{z_{\alpha}\left(\alpha_{i}\right)} \dot{z}_{i}\right)\right] .
$$

Therefore, we obtain

$$
\begin{aligned}
\left(I-i H_{h}\right) \dot{\gamma}_{i}= & \left(I-i H_{h}\right)\left[2 D_{h} \dot{\phi}_{i}-2 \operatorname{Re}\left(w_{0}\left(\alpha_{i}\right) D_{h} \dot{z}_{i}\right)+i \operatorname{Im}\left(\frac{\gamma\left(\alpha_{i}\right)}{z_{\alpha}\left(\alpha_{i}\right)} D_{h}\left(\dot{z}_{i}\right)\right)\right] \\
& +A_{0}\left(\dot{z}_{i}\right)+A_{0}\left(\dot{\phi}_{i}\right)+O\left(h^{r}\right),
\end{aligned}
$$

where we have used Lemma 1. Now substituting (61), (62), and (64) into (53), we get

$$
\begin{aligned}
\frac{d \dot{z}_{i}^{*}}{d t}= & \frac{1}{2 z_{\alpha}\left(\alpha_{i}\right)}\left(I-i H_{h}\right) \\
& \cdot\left[2 D_{h} \dot{\phi}_{i}-2 \operatorname{Re}\left(w_{0}\left(\alpha_{i}\right) D_{h} \dot{z}_{i}\right)+i \operatorname{Im}\left(\frac{\gamma\left(\alpha_{i}\right)}{z_{\alpha}\left(\alpha_{i}\right)} D_{h} \dot{z}_{i}\right)-\frac{\gamma\left(\alpha_{i}\right)}{z_{\alpha}\left(\alpha_{i}\right)} D_{h} \dot{z}_{i}\right] \\
& +A_{0}\left(\dot{z}_{i}\right)+A_{0}\left(\dot{\phi}_{i}\right)+O\left(h^{r}\right) .
\end{aligned}
$$

Note that

$$
\begin{aligned}
& -2 \operatorname{Re}\left(w_{0}\left(\alpha_{i}\right) D_{h} \dot{z}_{i}\right)+i \operatorname{Im}\left(\frac{\gamma\left(\alpha_{i}\right)}{z_{\alpha}\left(\alpha_{i}\right)} D_{h} \dot{z}_{i}\right)-\frac{\gamma\left(\alpha_{i}\right)}{z_{\alpha}\left(\alpha_{i}\right)} D_{h} \dot{z}_{i} \\
& =-2 \operatorname{Re}\left(w_{0}\left(\alpha_{i}\right) D_{h} \dot{z}_{i}\right)-\operatorname{Re}\left(\frac{\gamma\left(\alpha_{i}\right)}{z_{\alpha}\left(\alpha_{i}\right)} D_{h} \dot{z}_{i}\right) \\
& =-2 \operatorname{Re}\left[\left(w_{0}\left(\alpha_{i}\right)+\frac{\gamma\left(\alpha_{i}\right)}{2 z_{\alpha}\left(\alpha_{i}\right)}\right) D_{h} \dot{z}_{i}\right]=2 \operatorname{Re}\left[w\left(\alpha_{i}\right) D_{h} \dot{z}_{i}\right],
\end{aligned}
$$

where $w\left(\alpha_{i}\right)=w_{0}\left(\alpha_{i}\right)+\left(\gamma\left(\alpha_{i}\right)\right) /\left(2 z_{\alpha}\left(\alpha_{i}\right)\right)$. Substituting (66) into (65) and using Lemma 1 again, we get

$$
\begin{aligned}
\frac{d \dot{z}^{*}}{d t} & =\frac{1}{z_{\alpha}\left(\alpha_{i}\right)}\left(I-i H_{h}\right)\left[D_{h} \dot{\phi}_{i}-\operatorname{Re}\left(w\left(\alpha_{i}\right) D_{h} \dot{z}_{i}\right)\right]+A_{0}\left(\dot{z}_{i}\right)+A_{0}\left(\dot{\phi}_{i}\right)+O\left(h^{r}\right) \\
& =\frac{1}{z_{\alpha}\left(\alpha_{i}\right)}\left(I-i H_{h}\right) D_{h}\left[\dot{\phi}_{i}-\operatorname{Re}\left(w\left(\alpha_{i}\right) \dot{z}_{i}\right)\right]+A_{0}\left(\dot{z}_{i}\right)+A_{0}\left(\dot{\phi}_{i}\right)+O\left(h^{r}\right)
\end{aligned}
$$


It is natural to set

$$
\dot{F}_{i}=\dot{\phi}_{i}-\operatorname{Re}\left(w\left(\alpha_{i}\right) \dot{z}_{i}\right)=\dot{\phi}_{i}-u\left(\alpha_{i}\right) \dot{x}_{i}-v\left(\alpha_{i}\right) \dot{y}_{i}
$$

Then (67) becomes

$$
\frac{d \dot{z}_{i}^{*}}{d t}=\frac{1}{z_{\alpha}\left(\alpha_{i}\right)}\left(I-i H_{h}\right) D_{h} \dot{F}_{i}+A_{0}\left(\dot{z}_{i}\right)+A_{0}\left(\dot{F}_{i}\right)+O\left(h^{r}\right) .
$$

We can also write (69) as

$$
\frac{d}{d t}\left(z_{\alpha}\left(\alpha_{i}\right) \dot{z}_{i}^{*}\right)=\left(I-i H_{h}\right) D_{h} \dot{F}_{i}+A_{0}\left(\dot{z}_{i}\right)+A_{0}\left(\dot{F}_{i}\right)+O\left(h^{r}\right) .
$$

Note that

$$
z_{\alpha}\left(\alpha_{i}\right) \dot{z}_{i}^{*}=\left(x_{\alpha}\left(\alpha_{i}\right) \dot{x}_{i}+y_{\alpha}\left(\alpha_{i}\right) \dot{y}_{i}\right)-i\left(-y_{\alpha} \dot{x}_{i}+x_{\alpha} \dot{y}_{i}\right)
$$

We introduce the normal and tangential vectors of the interface position as

$$
\begin{aligned}
& \vec{N}(\alpha)=\frac{\left(-y_{\alpha}, x_{\alpha}\right)}{\sqrt{x_{\alpha}^{2}+y_{\alpha}^{2}}}=\sigma(\alpha)\left(-y_{\alpha}, x_{\alpha}\right), \\
& \vec{T}(\alpha)=\frac{\left(x_{\alpha}, y_{\alpha}\right)}{\sqrt{x_{\alpha}^{2}+y_{\alpha}^{2}}}=\sigma(\alpha)\left(x_{\alpha}, y_{\alpha}\right),
\end{aligned}
$$

where $\sigma=\left(x_{\alpha}^{2}+y_{\alpha}^{2}\right)^{-1 / 2}$. We define

$$
\dot{x}^{T}=(\dot{x}, \dot{y}) \cdot \vec{T}, \quad \dot{x}^{N}=(\dot{x}, \dot{y}) \cdot \vec{N} .
$$

Then we obtain

$$
z_{\alpha}\left(\alpha_{i}\right) \dot{z}_{i}^{*}=\frac{1}{\sigma\left(\alpha_{i}\right)}\left[\dot{x}_{i}^{T}-i \dot{x}_{i}^{N}\right]
$$

Substituting (74) into (70), we get

$$
\begin{aligned}
& \left(\dot{x}_{i}^{T}\right)_{t}=\sigma\left(\alpha_{i}\right) D_{h} \dot{F}_{i}+A_{0}\left(\dot{x}_{i}^{T}\right)+A_{0}\left(\dot{x}_{i}^{N}\right)+A_{0}\left(\dot{F}_{i}\right)+O\left(h^{r}\right), \\
& \left(\dot{x}_{i}^{N}\right)_{t}=\sigma\left(\alpha_{i}\right) H_{h} D_{h} \dot{F}_{i}+A_{0}\left(\dot{x}_{i}^{T}\right)+A_{0}\left(\dot{x}^{N}\right)+A_{0}\left(\dot{F}_{i}\right)+O\left(h^{r}\right) .
\end{aligned}
$$

We introduce a change of variables

$$
\dot{\delta}_{i}=\dot{x}_{i}^{T}+H_{h} \dot{x}_{i}^{N}, \quad \dot{x}_{i}^{N}=\dot{x}_{i}^{N} .
$$

Then (75), (76) are reduced to

$$
\begin{aligned}
\left(\dot{x}_{i}^{N}\right)_{t} & =\sigma\left(\alpha_{i}\right) H_{h} D_{h} \dot{F}_{i}+A_{0}\left(\dot{x}_{i}^{T}, \dot{x}_{i}^{N}\right)+A_{0}\left(\dot{F}_{i}\right)+O\left(h^{r}\right), \\
\left(\dot{\delta}_{i}\right)_{t} & =A_{0}\left(\dot{x}_{i}^{T}, \dot{x}_{i}^{N}\right)+A_{0}\left(\dot{F}_{i}\right)+0\left(h^{r}\right),
\end{aligned}
$$

where $A_{0}\left(\dot{x}_{i}^{T}, \dot{x}_{i}^{N}\right)=A_{0}\left(\dot{x}_{i}^{T}\right)+A_{0}\left(\dot{x}_{i}^{N}\right)$. We still need to express the evolution equation for $\dot{F}_{i}$ in the new variables. From the definition (68), we have

$$
\left(\dot{F}_{i}\right)_{t}=\left(\dot{\phi}_{i}\right)_{t}-u\left(\alpha_{i}\right) \dot{u}_{i}-v\left(\alpha_{i}\right) \dot{v}_{i}-u_{t}\left(\alpha_{i}\right) \dot{x}_{i}-v_{t}\left(\alpha_{i}\right) \dot{y}_{i},
$$


where

$$
\dot{u}_{i}=u_{i}-u\left(\alpha_{i}\right), \quad \dot{v}_{i}=v_{i}-v\left(\alpha_{i}\right), \quad u_{i}=\frac{d x_{i}}{d t}, \quad v_{i}=\frac{d y_{i}}{d t} .
$$

To evaluate $\left(\dot{\phi}_{i}\right)_{t}$, we compare (8) with the continuous Bernoulli law, i.e.,

$$
\begin{gathered}
\frac{d \phi_{i}}{d t}=\frac{1}{2}\left(u_{i}^{2}+v_{i}^{2}\right)-g y_{i}, \\
\frac{d \phi\left(\alpha_{i}\right)}{d t}=\frac{1}{2}\left(u\left(\alpha_{i}\right)^{2}+v\left(\alpha_{i}\right)^{2}\right)-g y\left(\alpha_{i}\right) .
\end{gathered}
$$

Subtracting (82) from (81), we obtain

$$
\left(\dot{\phi}_{i}\right)_{t}=\frac{1}{2}\left(u_{i}^{2}-u\left(\alpha_{i}\right)^{2}\right)+\frac{1}{2}\left(v_{i}^{2}-v\left(\alpha_{i}\right)^{2}\right)-g \dot{y}_{i}
$$

or, equivalently,

$$
\left(\dot{\phi}_{i}\right)_{t}=u\left(\alpha_{i}\right) \dot{u}_{i}+v\left(\alpha_{i}\right) \dot{v}_{i}-g \dot{y}_{i}+\frac{1}{2}\left(\dot{u}_{i}^{2}+\dot{v}_{i}^{2}\right) .
$$

Substituting (84) into (80), we obtain after cancellation

$$
\left(\dot{F}_{i}\right)_{t}=-u_{t}\left(\alpha_{i}\right) \dot{x}_{i}-\left(v_{t}\left(\alpha_{i}\right)+g\right) \dot{y}_{i}+\frac{1}{2}\left(\dot{u}_{i}^{2}+\dot{v}_{i}^{2}\right)
$$

It is important that the right-hand side of (85) depends only on the normal component of the vector $(\dot{x}, \dot{y})$ to the leading order. To see this, we note that the Lagrangian velocity $(u, v)$ satisfies the Euler equations

$$
u_{t}+p_{x}=0, \quad v_{t}+p_{y}+g=0,
$$

so that

$$
-\left(u_{t}, v_{t}+g\right)=\left(p_{x}, p_{y}\right)
$$

Moreover, $p=0$ on the interface so that $\nabla p$ is in the normal direction. We have

$$
-u_{t} \dot{x}_{i}-\left(v_{t}+g\right) \dot{y}_{i}=\left(p_{x}, p_{y}\right) \cdot\left(\dot{x}_{i}, \dot{y}_{i}\right) \equiv-c\left(\alpha_{i}\right) \dot{x}_{i}^{N},
$$

where

$$
c\left(\alpha_{i}\right)=-\nabla p\left(\alpha_{i}\right) \cdot \vec{N}_{i}=\left(u_{t}, v_{t}+g\right) \cdot \vec{N}
$$

Therefore, we get

$$
\left(\dot{F}_{i}\right)_{t}=-c\left(\alpha_{i}\right) \dot{x}_{i}^{N}+\frac{1}{2}\left(\dot{u}_{i}^{2}+\dot{v}_{i}^{2}\right)
$$

Now the whole set of evolution equations for $\dot{x}^{N}, \dot{\delta}$, and $\dot{F}$ is

$$
\begin{aligned}
\left(\dot{x}_{i}^{N}\right)_{t} & =\sigma\left(\alpha_{i}\right) H_{h} D_{h} \dot{F}_{i}+A_{0}\left(\dot{x}_{i}^{N}, \dot{\delta}_{i}\right)+A_{0}\left(\dot{F}_{i}\right)+O\left(h^{r}\right), \\
\left(\dot{\delta}_{i}\right)_{t} & =A_{0}\left(\dot{x}_{i}^{N}, \dot{\delta}_{i}\right)+A_{0}\left(\dot{F}_{i}\right)+O\left(h^{r}\right), \\
\left(\dot{F}_{i}\right)_{t} & =-c\left(\alpha_{i}\right) \dot{x}_{i}^{N}+\frac{1}{2}\left(\dot{u}_{i}^{2}+\dot{v}_{i}^{2}\right) .
\end{aligned}
$$


Thus, we have reduced the error estimates for the full nonlinear, nonlocal water wave equations into a simple linear and almost-local system for the variation quantities. The only nonlocality in the leading-order terms comes from the discrete Hilbert transform $H_{h}$ in (89). The lowerorder terms are nonlocal, but they are smoother than the principal linearized terms. This simplification helps us identify and balance the most important terms in our energy estimates. Define a discrete operator

$$
\Lambda_{h}=H_{h} D_{h}
$$

It is important to note that $\Lambda_{h}$ is a positive and symmetric operator. In fact, $\Lambda_{h}$ has the discrete Fourier symbol $|k| \rho(k h)$. We introduce a discrete $H^{1 / 2}$ norm as

$$
\|f\|_{H_{h}^{1 / 2}}=\left(\left(\Lambda_{h}+1\right) f, f\right)^{1 / 2} .
$$

We also assume as in hypothesis (11) of Theorem 1 that $c(\alpha)$ is positive:

$$
c(\alpha) \geq c_{0}>0 .
$$

To perform energy estimates, we multiply (89) by $\left(c\left(\alpha_{i}\right)\right) /\left(\sigma\left(\alpha_{i}\right)\right) \dot{x}_{i}^{N},(90)$ by $\dot{\delta}_{i}$, and (91) by $\left(\Lambda_{h}+1\right) \dot{F}_{i}$, sum in $i$, then add up. With

$$
y_{0}(t)^{2}=\left\|\left(\frac{c}{\sigma}\right)^{1 / 2} \dot{x}^{N}\right\|_{l^{2}}^{2}+\|\dot{\delta}\|_{l^{2}}^{2}+\|\dot{F}\|_{H_{h}^{1 / 2}}^{2},
$$

the result is

$$
\begin{aligned}
\frac{1}{2} \frac{d}{d t} y_{0}^{2}(t)= & \left(\Lambda_{h} \dot{F}, c \dot{x}^{N}\right)-\left(c \dot{x}^{N},\left(\Lambda_{h}+1\right) \dot{F}\right) \\
& +\left(f^{(1)}, x^{N}\right)+\left(f^{(2)}, \dot{\delta}\right)+\left(f^{(3)},\left(\Lambda_{h}+1\right) \dot{F}\right),
\end{aligned}
$$

where

$$
\begin{gathered}
\left\|f^{(j)}\right\|_{l^{2}} \leq c\left(\left\|\dot{x}^{N}\right\|_{l^{2}}+\|\dot{\delta}\|_{l^{2}}+\|\dot{F}\|_{l^{2}}+h^{r}\right) \text { for } j=1,2, \\
f_{i}^{(3)}=\frac{1}{2}\left(\dot{u}_{i}^{2}+\dot{v}_{i}^{2}\right) .
\end{gathered}
$$

By (69), we have

$$
\dot{u}_{j}-i \dot{v}_{j}=\frac{d \dot{z}_{j}^{*}}{d t}=\frac{1}{z_{\alpha}\left(\alpha_{j}\right)}\left(I-i H_{h}\right) D_{h} \dot{F}_{j}+A_{0}\left(\dot{z}_{j}\right)+A_{0}\left(\dot{F}_{j}\right)+O\left(h^{r}\right) .
$$

Arguing as in (41) we can show, using (40), that

$$
\left\|D_{h} \dot{\phi}\right\|_{\infty} \leq 2 \pi h .
$$

Thus, it follows from (40), (68) that for $t \leq T^{*}$,

$$
\|\dot{u}\|_{\infty}+\|\dot{v}\|_{\infty} \leq c h .
$$

Therefore, we have

$$
\begin{aligned}
\left.\mid\left(\Lambda_{h}+1\right) \dot{F}, \dot{u}^{2}+\dot{v}^{2}\right) \mid & \leq\left\|\left(\Lambda_{h}+1\right) \dot{F}\right\|_{l^{2}}\left\|\dot{u}^{2}+\dot{v}^{2}\right\|_{l^{2}} \\
& \leq\left\|\left(\Lambda_{h}+1\right) \dot{F}\right\|_{l^{2}} \operatorname{ch}\left(\left\|D_{h} \dot{F}\right\|_{l^{2}}+\|\dot{F}\|_{l^{2}}+\|\dot{z}\|_{l^{2}}+h^{r}\right) .
\end{aligned}
$$


Note that

$$
\begin{aligned}
\left\|\left(\Lambda_{h}+1\right) \dot{F}\right\|_{l^{2}} & =\left(\sum_{k=-N / 2+1}^{N / 2}(|k| \rho(k h)+1)^{2}\left|\hat{\dot{F}}_{k}\right|^{2}\right)^{1 / 2} \\
& \leq \sqrt{N / 2+1}\left(\sum_{|k| \leq N / 2}(|k| \rho(k h)+1)\left|\hat{\dot{F}}_{k}\right|^{2}\right)^{1 / 2} \leq C h^{-1 / 2} \mid \dot{F} \|_{H_{h}^{1 / 2}} .
\end{aligned}
$$

Similarly, we can show

$$
\left\|D_{h} \dot{F}\right\|_{l^{2}} \leq C h^{-1 / 2}\|\dot{F}\|_{H_{h}^{1 / 2}}
$$

As a consequence,

$$
\left|\left(\left(\Lambda_{h}+1\right) \dot{F}, \dot{u}^{2}+\dot{v}^{2}\right)\right| \leq C\|\dot{F}\|_{H_{h}^{1 / 2}}\left(\|\dot{F}\|_{H_{h}^{1 / 2}}+\left\|\dot{x}^{N}\right\|_{l^{2}}+\|\dot{\delta}\|_{l^{2}}+h^{r}\right) .
$$

Hence, we obtain

$$
\frac{1}{2} \frac{d}{d t} y_{0}^{2}(t) \leq C\left(y_{0}^{2}+y_{0} h^{r}\right)
$$

The Gronwall inequality then implies

$$
y_{0}(t) \leq C(T) h^{r}, \quad t \leq T^{*} .
$$

In terms of the original variables, we have

$$
\|\dot{z}\|_{l^{2}}+\|\dot{\phi}\|_{l^{2}} \leq B(T) h^{r}, \quad\|\dot{\gamma}\|_{l^{2}} \leq B(T) h^{r-1}, \quad t \leq T^{*},
$$

where we have used formula (60) for $\dot{\gamma}$. For $m \geq 3$, and $h$ small, we get

$$
\|\dot{z}\|_{l^{2}}+\|\dot{\phi}\|_{l^{2}} \leq B(T) h^{3}<\frac{1}{2} h^{5 / 2}, \quad\|\dot{\gamma}\|_{l^{2}}<\frac{1}{2} h^{3 / 2} .
$$

It follows from the definition of $T^{*}$ in (40) that

$$
T^{*}=T \text {. }
$$

This implies that estimate (104) is valid for the entire time interval $0 \leq t \leq T$. Convergence of the interface velocity $w$ follows from (98). This completes the convergence proof of our scheme.

5. The case with surface tension. In this section, we consider the case with surface tension. As before, we assume that the fluid occupies the lower region. As it was shown in [BHL1], in the case with surface tension, the direction of gravity plays no role in the stability estimates. Consequently, the analysis also applies to the case with fluid above the interface.

The effect of surface tension is to introduce a discontinuity in pressure across the interface proportional to the (mean) curvature. The pressure is larger below when the interface is curved toward the lower region. The earlier form of Bernoulli's equation (2) is now replaced by

$$
\phi_{t}=\frac{1}{2}\left(u^{2}+v^{2}\right)-g y+\tau \kappa
$$


Here $\kappa$ is the curvature and the constant $\tau$ is the coefficient of surface tension. The new set of evolution equations now becomes

$$
\begin{aligned}
z_{t}^{*} & =\frac{1}{4 \pi i} \int_{-\pi}^{\pi} \gamma\left(\alpha^{\prime}\right) \cot \left(\frac{z(\alpha)-z\left(\alpha^{\prime}\right)}{2}\right) d \alpha^{\prime}+\frac{1}{2} \frac{\gamma(\alpha)}{z_{\alpha}(\alpha)} \equiv w(\alpha, t), \\
\phi_{t} & =\frac{1}{2}\left(u^{2}+v^{2}\right)-g y+\tau \kappa, \\
\phi_{\alpha} & =\frac{\gamma}{2}+\operatorname{Re}\left[\frac{z_{\alpha}}{4 \pi i} \int_{-\pi}^{\pi} \gamma\left(\alpha^{\prime}\right) \cot \left(\frac{z(\alpha)-z\left(\alpha^{\prime}\right)}{2}\right) d \alpha^{\prime}\right] .
\end{aligned}
$$

The curvature $\kappa$ can be expressed by

$$
\kappa=\frac{x_{\alpha} y_{\alpha \alpha}-y_{\alpha} x_{\alpha \alpha}}{\left(x_{\alpha}^{2}+y_{\alpha}^{2}\right)^{3 / 2}}
$$

In the case with surface tension, we need to use the sharper estimate (26) in Lemma 2. This is because $\phi$ now contains second-order derivatives of $z$. In order to close the energy estimates, we require an $A_{-1}(\dot{\phi})$ in (65) or, equivalently, $A_{-1}(\dot{F})$ in (69). This will be seen in the proof. This amounts to having better control of the aliasing errors arising from the alternatingpoint vortex quadrature rule. This requirement will be satisfied if we impose, in addition, that $\rho^{\prime}( \pm \pi)=0$. For the spectral derivative approximation, we can always require that the Fourier smoothing multiplier $\rho(x)$ satisfy this property. The numerical approximation for (105)-(107) is given by

$$
\begin{aligned}
\frac{d z_{i}^{*}}{d t} & =\frac{1}{4 \pi i} \sum_{(j-i) \text { odd }} \gamma_{j} \cot \left(\frac{z_{i}^{p}-z_{j}^{p}}{2}\right)(2 h)+\frac{\gamma_{i}}{2\left(D_{h} z_{i}\right)} \equiv w_{i}=u_{i}-i v_{i}, \\
\frac{d \phi_{i}}{d t} & =\frac{1}{2}\left(u_{i}^{2}+v_{i}^{2}\right)-g y_{i}+\tau \kappa_{i}, \\
D_{h} \phi_{i} & =\frac{\gamma_{i}}{2}+\operatorname{Re}\left[\frac{D_{h} z_{i}}{4 \pi i} \sum_{(j-i) \text { odd }} \gamma_{j} \cot \left(\frac{z_{i}^{p}-z_{j}^{p}}{2}\right) 2 h\right], \\
\kappa_{i} & =\frac{D_{h} x_{i}^{q} D_{h}^{2} y_{i}-D_{h} y_{i}^{q} D_{h}^{2} x_{i}}{\left(\left(D_{h} x_{i}^{q}\right)^{2}+\left(D_{h} y_{i}^{q}\right)^{2}\right)^{3 / 2}}
\end{aligned}
$$

where $z^{p}=\alpha+s^{p}, z^{q}=\alpha+s^{q}, \hat{s}_{i}^{p}=\rho(k h) \hat{s}_{k}, \hat{s}_{i}^{q}=q(k h) \hat{s}_{k}$, and $q(x)=\frac{d}{d x}(x \rho(x))$.

We remark that the use of $z^{q}$ in (112) is to balance the high-mode error due to aliasing in the spectral approximation or in the finite-order accurate approximation. No additional smoothing is necessary for the second derivative terms $D_{h}^{2} x_{i}$ and $D_{h}^{2} y_{i}$. This is determined by the precise form of the discrete product rule

$$
D_{h}(f z)=f D_{h} z+\left(D_{h} f\right) z^{q}+h A_{0}(z)
$$

and

$$
D_{h}\left(f D_{h} z\right)=f D_{h}^{2} z+\left(D_{h} f\right) D_{h} z^{q}+A_{0}(z),
$$

as given by Lemma 1 . The $q$ smoothing on $z$ is necessary in obtaining estimate (121). Without this modification, the numerical discretization would be unstable for finite-order derivative operators. This can be verified by performing linear stability analysis (the normal mode analysis) around equilibrium solutions. 
Note that if $\rho(k h)$ corresponds to an $r$ th-order derivative, then

$$
|\rho(k h)-1| \leq C(k h)^{r}, \quad\left|\rho^{\prime}(k h)\right| \leq C(k h)^{r-1} .
$$

This implies that

$$
|q(k h)-1| \leq C(k h)^{r} .
$$

Hence, $z^{q}$ and $z^{p}$ are $r$ th-order approximations to $z$.

For finite-order approximations, the natural filtering associated with $D_{h}$ is not strong enough since $\rho^{\prime}(\pi) \neq 0$ for all finite-order derivative operators. Therefore, we need to apply an additional filtering before we take the finite-order derivative in equations (109) and (111). That is, we should replace $D_{h}$ by $\tilde{D}_{h}$ in (109) and (111), where $\tilde{D}_{h} z_{i}=D_{h} z_{i}^{s}$ and $\hat{z}_{k}^{s}=\hat{z}_{k} s(k h)$. The Fourier multiplier $s$ satisfies

$$
|1-s(k h)| \leq C(k h)^{r}, \quad s \geq 0, \quad \text { and } \quad s( \pm \pi)=0 .
$$

The derivative operators in (112) remain unchanged. To maintain the balances of highfrequency errors, we need to modify the filtering operator accordingly to retain the structure of the high-frequency errors we had before. That is, $\hat{z}_{k}^{p}$ now becomes $\rho(k h) s(k h) \hat{z}_{k}$. With these modifications, the algorithm is exactly the same as (109)-(112). We can prove the following result.

THEOREM 2. Assume that $z(\cdot, t), \phi(\cdot, t) \in C^{m+3}[0,2 \pi]$, and $\gamma(\cdot, t) \in C^{m+2}[0,2 \pi]$ for $t \leq T$ and $m \geq 4$. If $D_{h}$ corresponds to an $r$ th-order approximation $r \geq 4$, then we have

$$
\begin{gathered}
\|z(\cdot, t)-z(t)\|_{H_{h}^{1}} \leq C(T) h^{r}, \\
\|\phi(\cdot, t)-\phi(t)\|_{H_{h}^{1 / 2}} \leq C(T) h^{r}, \\
\|\gamma(\cdot, t)-\gamma(t)\|_{H_{h}^{1 / 2}} \leq C(T) h^{r-1},
\end{gathered}
$$

where

$$
\|\phi\|_{H_{h}^{1 / 2}}^{2}=\sum_{k=-N / 2+1}^{N / 2}(1+|k| \tilde{\rho}(k h))\left|\hat{\phi}_{k}\right|^{2}, \quad \tilde{\rho}(k h)=\rho(k h) s(k h),
$$

and

$$
\|\phi\|_{H_{h}^{1}}^{2}=\|\phi\|_{l^{2}}^{2}+\left\|D_{h} \phi\right\|_{l^{2}}^{2} .
$$

$\hat{\phi}_{k}$ is the discrete Fourier transform of $\phi_{i}$. If $D_{h}$ corresponds to the spectral derivative, the result is the same by replacing $r$ by $m$, and replacing $\tilde{\rho}$ by $\rho$.

Remark 1. Again, by using Strang's argument, we can improve the convergence result to the optimal rate $h^{r}$ in the case of finite-order derivative approximations. In that case, we will obtain convergence for the second-order discretization as well.

Remark 2. In the presence of surface tension, the right-hand sides of the equations involve higher-order derivatives of the interface variables. Therefore, we have to work with a high-order norm to obtain stability. Consequently, the accuracy is one order lower than the order of accuracy for the case without surface tension.

To illustrate how the algorithm looks in a concrete example, we consider the case of the second-order finite difference approximation $D_{h}$ :

$$
D_{h} f_{i}=\frac{f_{i+1}-f_{i-1}}{2 h} \text {. }
$$


The corresponding $\rho(k h)$ is given by $\rho(x)=\frac{\sin (x)}{x}$, so that $q(x)=\cos x$. Clearly, we have

$$
\hat{z}_{k}^{q}=\cos (k h) \hat{z}_{k}=\left(\frac{e^{-i k h}+e^{i k h}}{2}\right) \hat{z}_{k},
$$

which implies that

$$
z_{i}^{q}=\frac{z_{i+1}+z_{i-1}}{2}
$$

As a result, we obtain

$$
D_{h} z_{i}^{q}=\frac{z_{i+2}-z_{i-2}}{4 h}=D_{2 h} z_{i}
$$

and

$$
D_{h}^{2} z_{i}=\frac{z_{i+2}-2 z_{i}+z_{i-2}}{4 h^{2}}
$$

This simply says that we should use every other gridpoint when discretizing the curvature $\kappa$.

Proof of Theorem 2. We take the spectral derivative approximation as an example. The consistency and the stability are almost the same as before. The only difference is in the way we treat the linear stability. Moreover, since the evolution equation for $z$ is the same as before, we have from (89) and (90) that

$$
\begin{aligned}
\left(\dot{x}_{i}^{N}\right)_{t}= & \sigma\left(\alpha_{i}\right) H_{h} D_{h} \dot{F}_{i}+A_{0}\left(\dot{x}_{i}^{N}, \dot{\delta}_{i}\right)+A_{-1}\left(\dot{F}_{i}\right)+O\left(h^{r}\right), \\
& \left(\dot{\delta}_{i}\right)_{t}=A_{0}\left(\dot{x}_{i}^{N}, \dot{\delta}_{i}\right)+A_{-1}\left(\dot{F}_{i}\right)+O\left(h^{r}\right),
\end{aligned}
$$

where we have used (26) to replace $A_{0}(\dot{F})$ by $A_{-1}(\dot{F})$ since we now have $\rho^{\prime}( \pm \pi)=0$. In our nonlinear stability estimates, we need to modify the definition of $T^{*}$ in (40) as

$$
T^{*}=\sup \left\{t \mid t \leq T,\|\dot{z}\|_{l^{2}},\|\dot{\phi}\|_{l^{2}} \leq h^{7 / 2},\|\dot{\gamma}\|_{l^{2}} \leq h^{5 / 2}\right\} .
$$

With this modification, we can show that the nonlinear term of the type defined by (47) is of the order $A_{-1}(\dot{z})$ instead of $A_{0}(\dot{z})$ in the case without surface tension. More precisely, the nonlinear terms are of the form $h A_{0}(\dot{z})$. We still set

$$
\dot{F}_{i}=\dot{\phi}_{i}-u\left(\alpha_{i}\right) \dot{x}_{i}-v\left(\alpha_{i}\right) \dot{y}_{i}
$$

It follows from (91) and (106) that

$$
\left(\dot{F}_{i}\right)_{t}=\tau \dot{\kappa}-c\left(\alpha_{i}\right) \dot{x}_{i}^{N}+\frac{1}{2}\left(\dot{u}_{i}^{2}+\dot{v}_{i}^{2}\right)
$$

where $\dot{\kappa}$ is defined by $\dot{\kappa}_{i}=\kappa_{i}-\kappa\left(\alpha_{i}\right)$. In the discrete case, the linear stability estimate for the curvature term is considerably more difficult than its continuous counterpart. This is due to the high-frequency error in the discrete derivative operator $D_{h}$. In the continuous case, we have the product rule

$$
D(f z)=f D z+(D f) z
$$

In the discrete case, we have from Lemma 1 that

$$
D_{h}(f z)=f D_{h} z+\left(D_{h} f\right) z^{q}+h A_{0}(z) .
$$


We define $P_{i}$ by

$$
P_{i} \equiv \frac{D_{h} x_{i}^{q} D_{h}^{2} y_{i}-D_{h} y_{i}^{q} D_{h}^{2} x_{i}}{\left(D_{h} x_{i}^{q}\right)^{2}+\left(D_{h} y_{i}^{q}\right)^{2}} .
$$

One crucial step in our estimates of the curvature term is to express the linear variation of $P_{i}$ by

$$
\dot{P}_{i}=D_{h}\left(\sigma\left(\alpha_{i}\right)^{2}\left(x_{\alpha}\left(\alpha_{i}\right) D_{h} \dot{y}_{i}-y_{\alpha}\left(\alpha_{i}\right) D_{h} \dot{x}_{i}\right)\right)+A_{-1}\left(\dot{z}_{i}\right),
$$

where $\sigma=\left(x_{\alpha}^{2}+y_{\alpha}^{2}\right)^{-1 / 2}$. It can be shown that without the $q$ smoothing in (119), estimate (120) is not valid. In proving (120), we have repeatedly used the discrete product rule (118). Using (120), we can show that the linear part of $\dot{\kappa}$ has the following simple expression in terms of the normal component of $\dot{z}$ :

$$
\dot{\kappa}=\sigma\left(\alpha_{i}\right) D_{h}\left[\sigma\left(\alpha_{i}\right) D_{h} \dot{x}_{i}^{N}\right]+A_{0}(\dot{z}) .
$$

Except for estimate (120), the proof of (121) is similar in spirit to the proof of the corresponding continuum case given in [BHL1]. Since the calculations are quite technical, we omit the proof here. Putting (115), (116), (117), and (121) together, we arrive at

$$
\begin{aligned}
\left(\dot{x}_{i}^{N}\right)_{t} & =\sigma\left(\alpha_{i}\right) H_{h} D_{h} \dot{F}_{i}+A_{0}\left(\dot{x}_{i}^{N}, \dot{\delta}_{i}\right)+A_{-1}\left(\dot{F}_{i}\right)+O\left(h^{r}\right) \\
\left(\dot{\delta}_{i}\right)_{t} & =A_{0}\left(\dot{x}_{i}^{N}, \dot{\delta}_{i}\right)+A_{-1}\left(\dot{F}_{i}\right)+O\left(h^{r}\right) \\
\left(\dot{F}_{i}\right)_{t} & =\tau \sigma\left(\alpha_{i}\right) D_{h}\left[\sigma\left(\alpha_{i}\right) D_{h} \dot{x}_{i}^{N}\right]+A_{0}(\dot{z})-c\left(\alpha_{i}\right) \dot{x}_{i}^{N}+\frac{1}{2}\left(\dot{u}_{i}^{2}+\dot{v}_{i}^{2}\right)+O\left(h^{r}\right) .
\end{aligned}
$$

One should note that the leading-order contribution in (124) comes from the surface tension. The term involving $c\left(\alpha_{i}\right)$ is a lower-order term here. Therefore, it plays no role in the leading-order stability analysis. This is in contrast to the case without surface tension where the sign of $c(\alpha)$ is crucial for determining the linear stability of the numerical scheme.

To perform energy estimates, we seek to balance the leading-order terms from (122) and (124). To this end, we first apply $D_{h}$ to both sides of (122), then multiply the resulting equation by $\tau \sigma\left(\alpha_{i}\right) D_{h} \dot{x}_{i}^{N}$, and sum in $i$. On the other hand, in (124), we multiply $\Lambda_{h} \dot{F}_{i}$ and sum in $i$. We then add these two equations. After summation by parts, the leading-order terms cancel each other, i.e.,

$$
\begin{aligned}
& \tau\left(\sigma D_{h} \dot{x}^{N}, D_{h} \sigma \Lambda_{h} \dot{F}\right)+\tau\left(\sigma D_{h}\left(\sigma D_{h} \dot{x}^{N}\right), \Lambda_{h} \dot{F}\right) \\
& =\tau\left(\sigma D_{h} \dot{x}^{N}, D_{h} \sigma \Lambda_{h} \dot{F}\right)-\tau\left(\left(\sigma D_{h} \dot{x}^{N}\right), D_{h} \sigma \Lambda_{h} \dot{F}\right)=0
\end{aligned}
$$

where we have used $\left(D_{h} f, g\right)=-\left(f, D_{h} g\right)$, which can be verified directly in Fourier space. Thus, we obtain

$$
\begin{aligned}
& \frac{d}{d t}\left(h \sum_{i=-N / 2+1}^{N / 2}\left(\sigma\left(\alpha_{i}\right)\left(D_{h} \dot{x}_{i}^{N}\right)^{2}+\left(\Lambda_{h} \dot{F}_{i}, \dot{F}_{i}\right)\right)\right. \\
& =\left(\tau \sigma\left(\alpha_{i}\right) D_{h} \dot{x}_{i}^{N}, D_{h} r_{1}\right)+\left(\Lambda_{h} \dot{F}_{i}, r_{2}\right)+R,
\end{aligned}
$$

where $r_{1}$ and $r_{2}$ are given by the lower-order terms in the right-hand side of (122) and (124), respectively. The time dependence of $\sigma$ also introduces a lower-order term $R$ which can be bounded in the same way as the lower-order terms $r_{1}$ and $r_{2}$. We neglect this time dependency here. Further, we multiply (122) by $\Lambda_{h} \dot{x}_{i}^{N}$, and sum in $i$. On the other hand, we multiply 
(124) by $\frac{1}{\tau \sigma} \dot{F}_{i}$ and sum in $i$. We then add these two equations. As before, the leading-order terms cancel out after summation by parts:

$$
\begin{aligned}
& \left(H_{h} D_{h} \dot{x}^{N}, \sigma H_{h} D_{h} \dot{F}\right)+\left(D_{h}\left(\sigma D_{h} \dot{x}^{N}\right), \dot{F}\right) \\
& =\left(\left(H_{h} D_{h} \dot{x}^{N}, H_{h} \sigma D_{h} \dot{F}\right)+\left(D_{h}\left(\sigma D_{h} \dot{x}^{N}\right), \dot{F}\right)\right) \\
& \quad-\left(\left(H_{h} D_{h} \dot{x}^{N},\left[H_{h}, \sigma\right] D_{h} \dot{F}\right)\right) \equiv R_{1}+R_{2},
\end{aligned}
$$

where $\left[H_{h}, \sigma\right]=H_{h} \sigma-\sigma H_{h}$ is the commutator operator. It has been shown in (38) that $\left[H_{h}, \sigma\right] z^{p}=A_{-1}(z)$. We have

$$
\left[H_{h}, \sigma\right] D_{h} \dot{F}=\left[H_{h}, \sigma\right]\left(S_{h} \dot{F}\right)^{p}=A_{-1}\left(S_{h} \dot{F}\right)=A_{0}(\dot{F}) .
$$

Therefore, we get

$$
\left|R_{2}\right| \leq\left\|H_{h} D_{h} \dot{x}^{N}\right\|_{l^{2}} \cdot\left\|\left[H_{h}, \sigma\right] D_{h} \dot{F}\right\|_{l^{2}} \leq C\left(\|\dot{F}\|_{l^{2}}^{2}+\left\|D_{h} \dot{x}^{N}\right\|_{l^{2}}^{2}\right) .
$$

As for $R_{1}$, note that $H_{h}^{2} D_{h}=-D_{h}$. Applying summation by parts, we get

$$
\begin{aligned}
R_{1} & =-\left(H_{h}^{2} D_{h} \dot{x}^{N}, \sigma D_{h} \dot{F}\right)+\left(D_{h}\left(\sigma D_{h} \dot{x}^{N}\right), \dot{F}\right) \\
& =\left(D_{h} \dot{x}^{N}, \sigma D_{h} \dot{F}\right)-\left(\sigma D_{h} \dot{x}^{N}, D_{h} \dot{F}\right)=0 .
\end{aligned}
$$

Therefore, we have

$$
\frac{d}{d t}\left[\left(\Lambda_{h} \dot{x}^{N}, \dot{x}^{N}\right)^{2}+\left(\frac{1}{\tau \sigma} \dot{F}, \dot{F}\right)\right]=\left(\Lambda_{h} \dot{x}^{N}, r_{1}\right)+\left(\frac{1}{\tau \sigma} \dot{F}, r_{2}\right) .
$$

Now we define

$$
\begin{aligned}
y_{0}(t)^{2}= & h \sum_{i=-N / 2+1}^{N / 2}\left(\sigma\left(\alpha_{i}\right)\left(D_{h} \dot{x}_{i}^{N}\right)^{2}+\left(\Lambda_{h} \dot{F}_{i}\right) \dot{F}_{i}+\left(\Lambda_{h} \dot{x}_{i}^{N}\right) \dot{x}_{i}^{N}+\frac{1}{\tau \sigma\left(\alpha_{i}\right)} F_{i}^{2}\right) \\
& +\left\|D_{h} \dot{\delta}\right\|_{l^{2}}^{2}+\left\|\dot{x}^{N}\right\|_{l^{2}}^{2}+\|\dot{\delta}\|_{l^{2}}^{2} .
\end{aligned}
$$

Then we can show that

$$
\frac{d}{d t} y_{0}(t)^{2} \leq C(T)\left[y_{0}(t)^{2}+h^{r} y_{0}(t)\right] .
$$

We remark that we do not lose any order of accuracy when we apply $D_{h}$ to (122)-(123) because the error $O\left(h^{r}\right)$ is of the form

$$
C_{r}\left(\alpha_{i}\right) h^{r}+C_{r+1}\left(\alpha_{i}\right) h^{r+1}+\cdots
$$

and the error coefficients are smooth. To prove (127), we need to estimate the lower-order contributions. We pick one term to illustrate the idea. To estimate $\left(\tau \sigma D_{h} \dot{x}^{N}, D_{h} r_{1}\right)$, we note that

$$
\left|\left(\tau \sigma D_{h} \dot{x}^{N}, D_{h} A_{-1}(\dot{F})\right)\right| \leq C\left(\left\|D_{h} \dot{x}^{N}\right\|_{l^{2}}^{2}+\|\dot{F}\|_{l^{2}}^{2}\right) .
$$

Recall that in our derivation, $A_{0}(\dot{z})$ comes from the two sources. One is from Lemma 1 when we use the discrete product rules. Another one comes from Lemma 4. From Lemma 1 we know that $A_{0}(\dot{z})=f_{\alpha} \dot{z}^{q}+h A_{0}(\dot{z})$. From the proof of Lemma 4 we have $A_{0}(\dot{z})=$ $D\left(\alpha_{i}\right) H_{h} \dot{z}+A_{-1}(\dot{z})$. In either case, we can easily show that

$$
\left\|D_{h} A_{0}(\dot{z})\right\|_{l^{2}} \leq C\left(\left\|D_{h} \dot{z}\right\|_{l^{2}}+\|\dot{z}\|_{l^{2}}\right) .
$$


Therefore, we have

$$
\left|\left(\tau \sigma D_{h} \dot{x}^{N}, D_{h} A_{0}\left(\dot{x}^{N}\right)\right)\right| \leq C\left(\left\|D_{h} \dot{x}^{N}\right\|_{l^{2}}^{2}+\left\|\dot{x}^{N}\right\|_{l^{2}}^{2}\right)
$$

and

$$
\left|\left(\Lambda_{h} \dot{F}_{i}, \dot{x}^{N}\right)\right| \leq\left|\left(H_{h} \dot{F}_{i}, D_{h} \dot{x}^{N}\right)\right| \leq\left\|D_{h} \dot{x}^{N}\right\|_{l^{2}}^{2}+\|\dot{F}\|_{l^{2}}^{2} .
$$

The other lower-order terms can be treated similarly. This proves (124). By Gronwall's inequality, we obtain

$$
y_{0}(t) \leq \tilde{C}(T) h^{r}
$$

The rest of the argument is the same as in the case without surface tension.

In the case of finite-order derivative operators, we need to modify the argument slightly. The derivative operator $D_{h}$ in (122) now becomes $\tilde{D}_{h}$, where $\left(\hat{\tilde{D}}_{h}\right)_{k}=i k \rho(k h) s(k h)$. Since we do not use $s$ smoothing in computing the curvature, the derivative operator $D_{h}$ in (124) is still the same, i.e., $\left(\hat{D}_{h}\right)_{k}=i k \rho(k h)$. We define $\tilde{\Lambda}_{h}=H_{h} \tilde{D}_{h}$.

To perform energy estimates, we seek to balance the leading-order terms from (122) and (124). As before, we first apply $D_{h}$ to both sides of (122), then multiply the resulting equation by $\tau \sigma\left(\alpha_{i}\right) D_{h} \dot{x}_{i}^{N}$, and sum in $i$. On the other hand, in (124), we multiply $\tilde{\Lambda}_{h} \dot{F}_{i}$ and sum in $i$. We then add these two equations. After summation by parts, the leading-order terms cancel each other, i.e.,

$$
\begin{aligned}
& \tau\left(\sigma D_{h} \dot{x}^{N}, D_{h} \sigma \tilde{\Lambda}_{h} \dot{F}\right)+\tau\left(\sigma D_{h}\left(\sigma D_{h} \dot{x}^{N}\right), \tilde{\Lambda}_{h} \dot{F}\right) \\
& =\tau\left(\sigma D_{h} \dot{x}^{N}, D_{h} \sigma \tilde{\Lambda}_{h} \dot{F}\right)-\tau\left(\left(\sigma D_{h} \dot{x}^{N}\right), D_{h} \sigma \tilde{\Lambda}_{h} \dot{F}\right)=0,
\end{aligned}
$$

where we have used $\left(D_{h} f, g\right)=-\left(f, D_{h} g\right)$. The rest of the argument is almost the same as in the case of using the spectral derivative. We omit the details here.

6. Numerical examples. In this section we present some numerical calculations that illustrate the performance of methods for which the convergence theory applies and also the difficulties which can arise from the stability issues that have been dealt with analytically. We present calculations of a standing wave, with or without surface tension, using the methods developed here. We use the same solution to illustrate that instabilities occur with finite-order derivatives if the filtering in $z$ is not used in the singular integrals. Finally, we present a calculation of a breaking wave without surface tension to demonstrate the behavior of the convergent method in the fully nonlinear regime.

In the standing wave calculations we use four different discretizations. In all cases, the velocity integral is discretized using the alternating trapezoidal rule. The only difference in these four schemes is the way in which the space derivative $\partial_{\alpha}$ is discretized. In the first scheme, we use a pseudospectral method. This scheme has been proved to be stable with the filtering of $z$. However, the filtering was not needed in the standing wave calculations because the solution is very smooth. The second scheme uses a second-order centered finite difference approximation for $\frac{\partial}{\partial \alpha}$; the third uses a fourth-order centered finite difference approximation for $\frac{\partial}{\partial \alpha}$. The fourth scheme uses a cubic spline approximation for $\frac{\partial}{\partial \alpha}$. We remark that the cubic splines have been widely used in boundary integral computations of interfacial flows; see, e.g., [BMO, Pull]. In our calculations, we choose a sinusoidal perturbation to the equilibrium solution of period 1. The initial condition is

$$
\begin{aligned}
& x(\alpha, 0)=\alpha+0.01 \sin (2 \pi \alpha), \quad y(\alpha, 0)=-0.01 \sin (2 \pi \alpha), \\
& \gamma(\alpha, 0)=0.01 \sin (2 \pi \alpha) .
\end{aligned}
$$




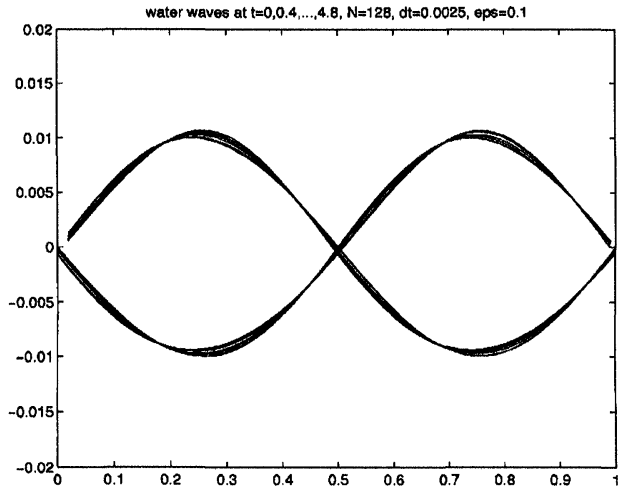

(a)

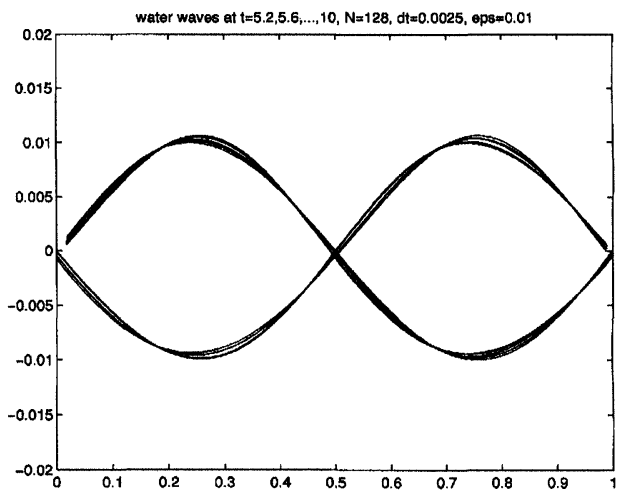

(b)

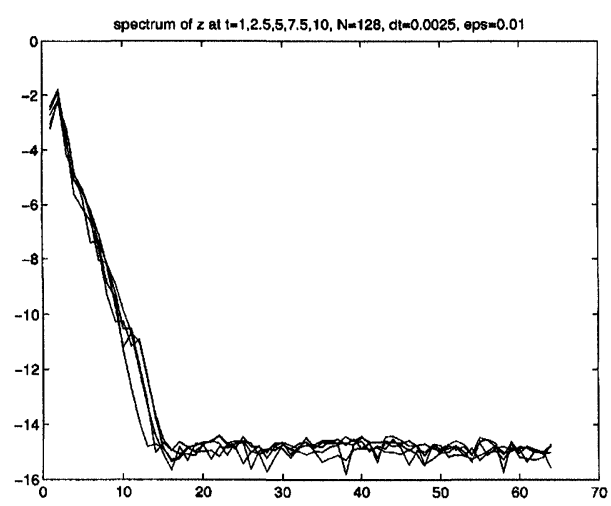

(c)

FIG. 1.

The gravity coefficient is set to 9.8 . This choice is not physically meaningful, but it leads to a predicted solution from the familiar linear theory of water waves which is a standing wave, periodic in time, with period .801 , without surface tension. A simple iterative scheme is used to solve for $\gamma$. The $(m+1)$ st iterative solution $\gamma^{m+1}$ is given by

$$
\gamma_{i}^{m+1}=2 D_{h} \phi_{i}-2 \operatorname{Re}\left[\frac{D_{h} z_{i}}{4 \pi i} \sum_{\substack{j=-N / 2+1 \\(j-i) \text { odd }}}^{N / 2} \gamma_{j}^{m} \cot \left(\frac{z_{i}^{p}-z_{j}^{p}}{2}\right) 2 h\right] .
$$

In our calculations presented in Figures 1-5, we use the fourth-order Runge-Kutta method as our time discretization. We use the solution of $\gamma$ at the previous time step as our initial guess for the iterative solution for $\gamma$. We stop the iteration when the error between the two consecutive iterative solutions is smaller than $10^{-10}$. For the calculations presented in Figures $1-5$, it typically takes about four iterations to converge.

We first use the pseudospectral method with no surface tension. In Figures $1 \mathrm{a}$ and $1 \mathrm{~b}$, we plot the numerical interface positions obtained from the first scheme from $t=0$ to $t=4.8$ and from $t=5.2$ to $t=10$, respectively. As predicted from the linear theory, we obtain a standing water wave with a period of about 0.8 time unit. So there are, in total, about 12.5 complete oscillations by time $t=10$. Clearly, the numerical solution is stable and smooth. It also suggests that there is a global smooth solution for the exact problem. In Figure 1c, the $\log$ of absolute $\hat{s}_{k}$ is plotted, where logs have base 10 . We can see that the round-off errors remain small at all times, indicating that no high-mode instability occurs in the calculation. 


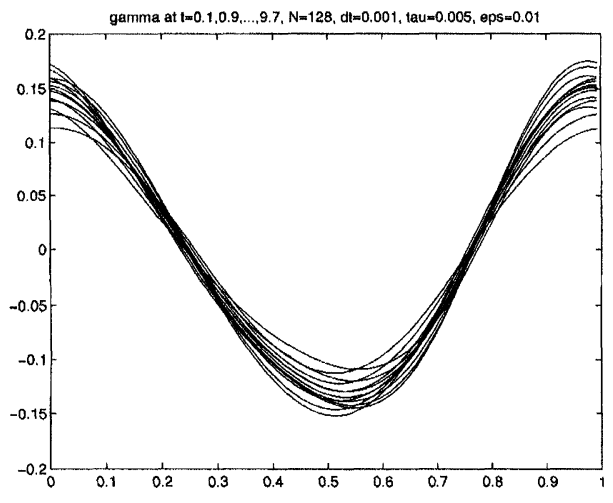

(a)

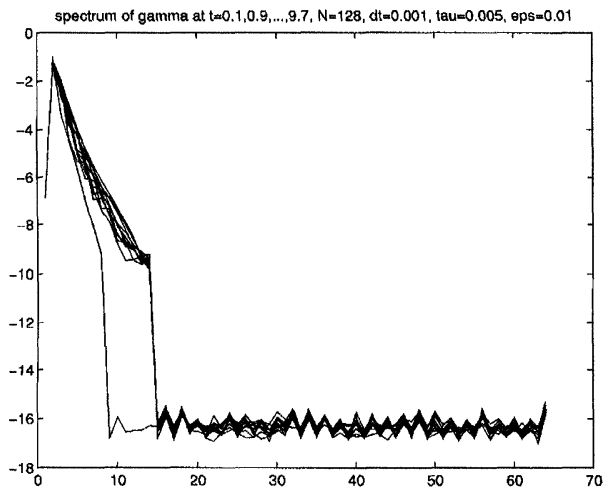

(b)

FIG. 2.

In Figures $2 \mathrm{a}$ and $2 \mathrm{~b}$ we present the calculation for the second-order derivative operator using the modified algorithm (109)-(112). The surface tension $\tau$ is equal to 0.005 . The period of oscillation predicted in the linear theory is shifted slightly to .792. Here it is important to use the $p$ and $q$ smoothing in (109)-(112) to obtain stability. We did not use the extra $s$ smoothing here because the solution is very smooth. We plot the vortex sheet strength and its Fourier spectrum. We have also performed calculations with more refined mesh sizes. The method is indeed stable. This is clear from Figure 2. Calculations with the fourth order and the cubic spline derivative operator, as well as the spectral derivative operator, also confirm our theoretical result. We do not present the pictures here since they are all very similar.

We remark that in the presence of surface tension there is an additional difficulty in time integration due to the stiffness of the resulting system. Surface tension introduces high-order spatial derivatives which are coupled to the interface equations in a nonlinear and nonlocal way. It is not straightforward how to apply implicit methods to remove the time step restrictions introduced by the surface tension. In [HLS], we present a new formulation of the interfacial problem that shows how to remove the strict time step restrictions. This reformation can be applied to a number of interfacial flows with surface tension, including the water wave problem considered here.

The calculations presented in Figures 3-5 are for the case with zero surface tension for finite-order derivative operators but without using the modification $z^{p}$ in the evaluation of the interface velocity. This is to illustrate that the additional filtering (i.e., $z^{p}$ ) is necessary. In Figure $3 \mathrm{a}$, we plot the numerical vortex sheet strength obtained from the second scheme at different times. We can see that there is a critical time beyond which the numerical solution starts to produce numerical oscillations. The oscillations grow rapidly with respect to time. If we further reduce the mesh size, the numerical oscillations will appear earlier. In Figure $3 b$, we examine the growth of the Fourier coefficients for the vortex sheet strength. We show the $\log$ plot of spectrum at different times ranging from $t=0.008$ to $t=0.22$. For $t$ small, we see that the spectrum decays exponentially. But due to numerical round-off errors, the computer cannot accurately represent the Fourier coefficients smaller than $10^{-16}$. These round-off errors are amplified by the numerical instability in time. As we can see, the round-off errors at the high modes are amplified the fastest. The ordering of the curves is in the increasing order of time. Only after the time $t=0.2$, do we begin to see the effect of this numerical instability in the physical space. Numerical oscillations start to grow rapidly in time. The highest $N$ mode does not grow, however, because the discrete derivative operator forces the highest mode to vanish. In our figures (except for Figure 5) for the Fourier spectrum, we did not plot the $\frac{N}{2}$ mode as it is identically zero. By comparison, the round-off error remains bounded at the 

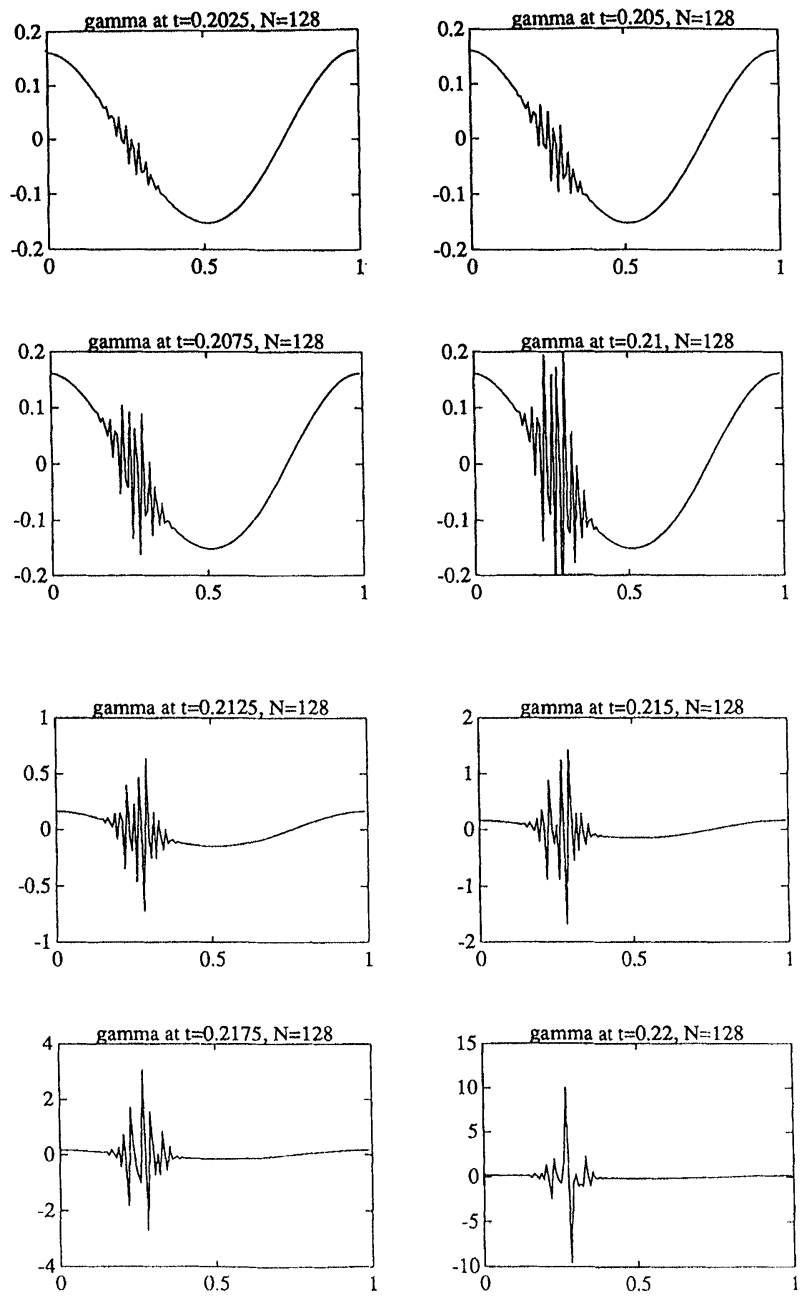

FIG. 3(a). Second-order finite difference derivative, water wave.

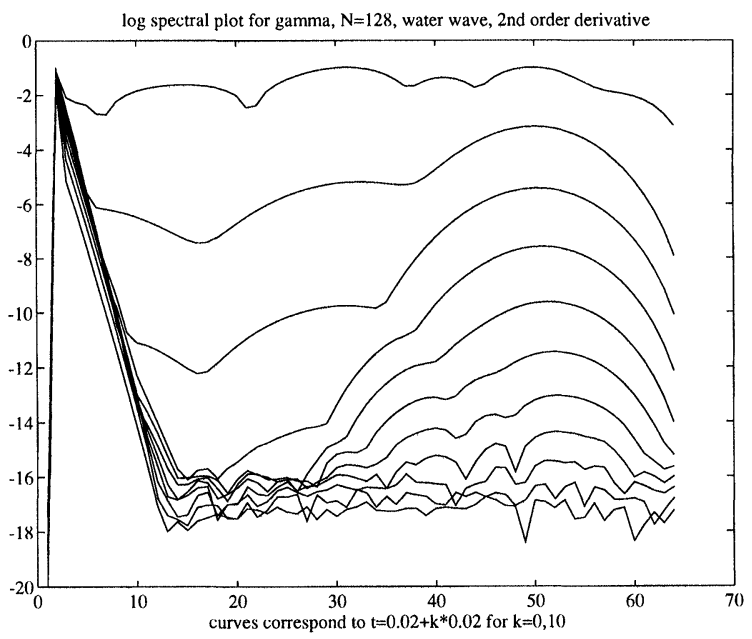

FIG. 3(b). 

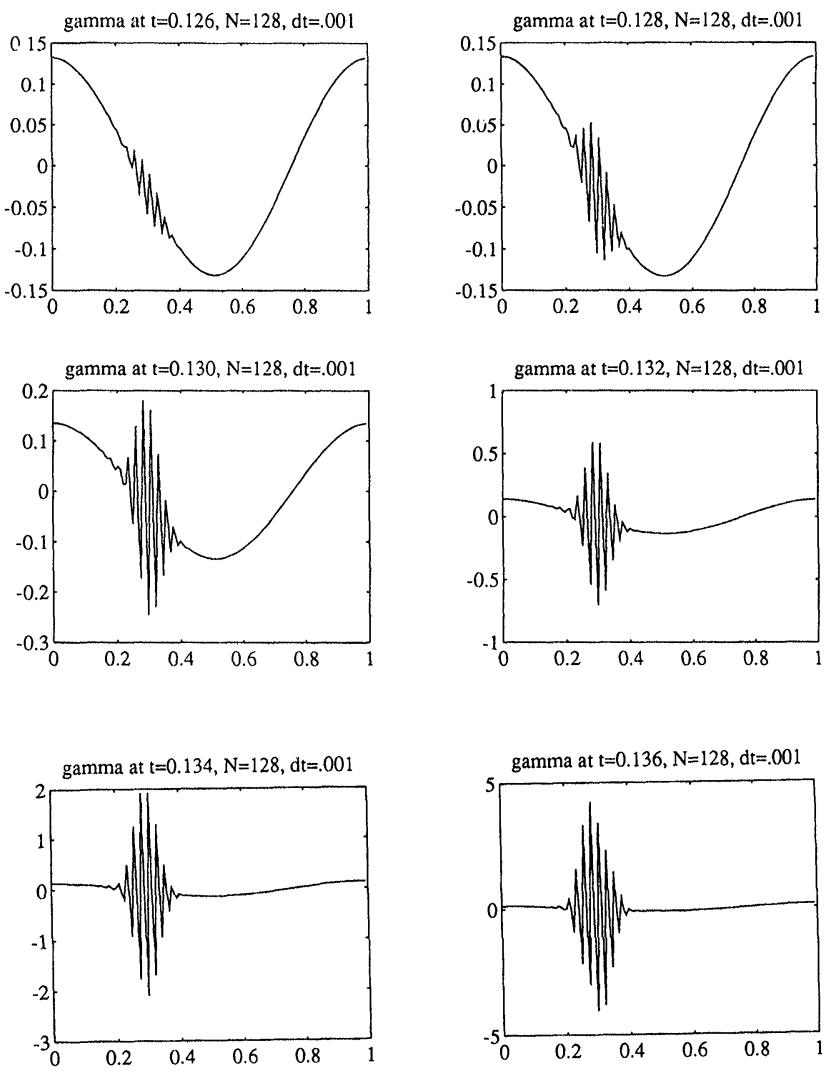

FIG. 4(a). Fourth-order finite difference derivative, water wave.

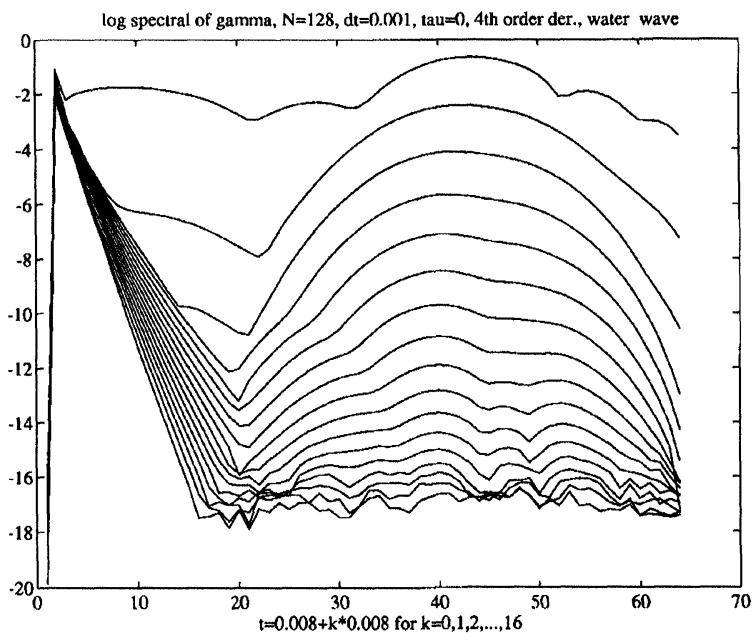

FIG. 4(b).

original round-off level for the first scheme; see Figure 1c. Very similar behavior is observed for the third and fourth schemes, except that the instability occurs earlier for the fourth-order scheme and the cubic spline approximation. The results are plotted in Figures $4 \mathrm{a}, 4 \mathrm{~b}$, and 5. We also include the pseudospectral calculation in Figure 5 for comparison. In Figure 5 the cubic spline is the solid line above and the spectral is the one below. 

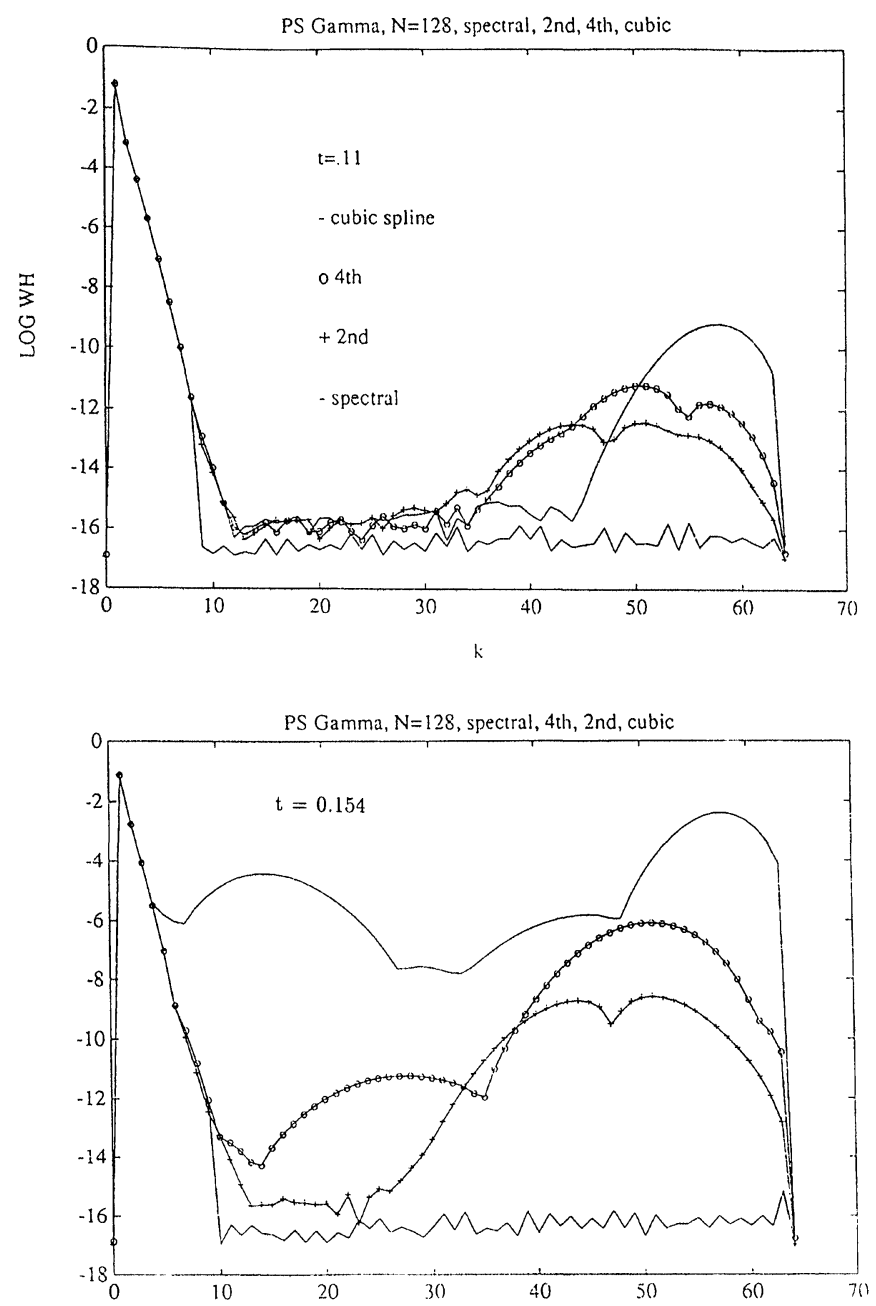

FIG, 5.

One can also obtain a more intuitive understanding of these numerical instabilities by performing a linearization around the equilibrium solution $z=\alpha, \gamma=0$, and $\phi=0$. For this choice of equilibrium solution, the corresponding interface velocity is equal to zero, i.e., $u=v=0$. The resulting linear equations for the variations reduce to a system of first-order partial differential equations with constant coefficients. Then, the usual normal mode analysis can be used to determine the linear stability of the various methods. We refer the reader to [BHL5] for details. Of course, it is not possible to analyze the aliasing errors (instability) from a near-equilibrium analysis as aliasing errors arise only in the case of nonconstant coefficients.

Next, we present a calculation of a breaking wave using our first scheme for zero surface tension. For a survey of breaking waves, see [Per]. In order to produce breaking in the water wave we use the following initial condition:

$$
\begin{aligned}
& x(\alpha, 0)=\alpha, \quad y(\alpha, 0)=0.1 \cos (2 \pi \alpha), \\
& \gamma(\alpha, 0)=1.0+0.1 \sin (2 \pi \alpha) .
\end{aligned}
$$

Note that the vortex sheet strength $\gamma$ does not have zero mean in this case. This amounts to a convenient choice of frame of reference. Although the derivation of (7)-(9) was for the 


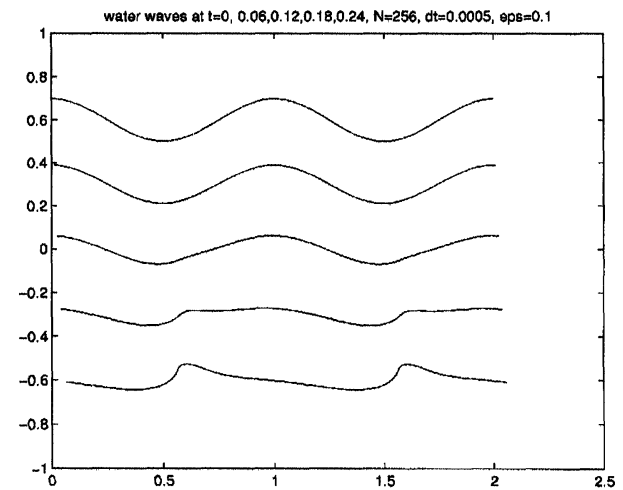

(a)

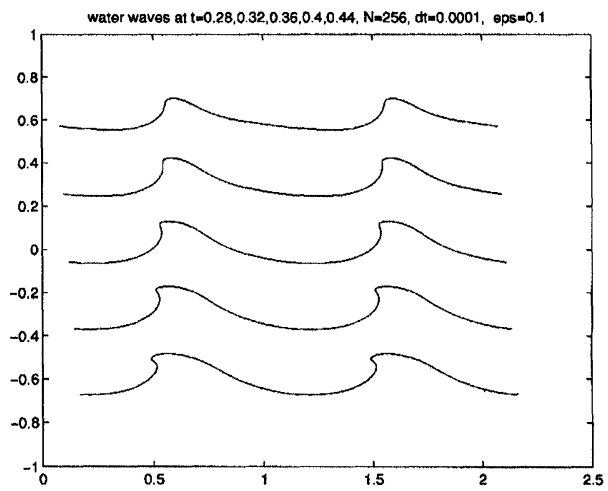

(b)

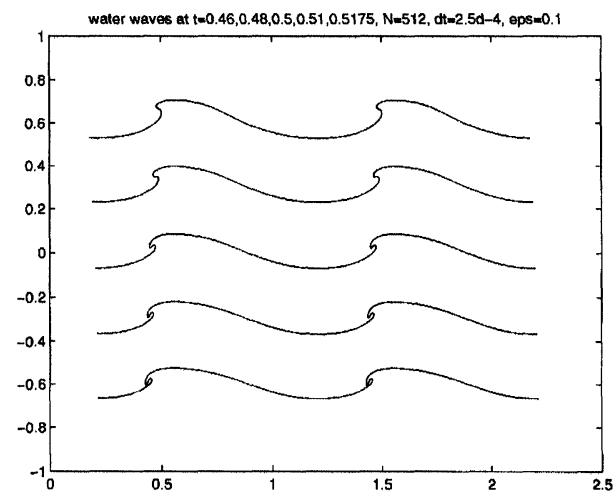

(c)

FIG. 6.

special case where $\phi$ is periodic and $\gamma$ has zero mean, the formulation is still valid provided that we only apply $D_{h}$ to equation (8); only $D_{h} \phi$ is needed in (9). Our numerical experiments have shown that a smaller perturbation of size 0.05 would give rise to an oscillatory wave which travels to the right and does not seem to break in finite time. The time integration in this numerical example is the fourth-order explicit Adams-Bashforth method. The fourthorder Runge-Kutta method is used to initialize the first three time steps. Also, a fourth-order extrapolation in time is used to obtain a more accurate initial guess for the iterative solution for $\gamma$ as suggested in [BMO]. With this improved initial guess, the iteration will converge with an iteration error of order $10^{-10}$ in two iterations for most time until the wave is close to breaking. In the calculation of breaking wave, we use a Fourier smoothing in the spectral derivative. The smoothing factor $\rho$ is chosen to be

$$
\rho(k h)=\exp \left(-10(2|k| / N)^{25}\right) \text { for }|k| \leq N / 2,
$$

so that $\left(\hat{D}_{h}\right)_{k}=i k \rho(k h)$ gives a 25 th-order accurate approximation to the derivative operator.

In Figures $6 \mathrm{a}-\mathrm{c}$ we present a series of interface profiles from $t=0$ to $t=0.5175$. In order to clearly see the time evolution of the water wave, we plot the solution at five different times in a single picture. The first curve from the top is obtained by adding 0.6 to the $y$ coordinate; the successive ones are displaced by multiples of 0.3. Time increases from top to bottom. As we can see from Figure 6b, the interface becomes vertical around $t=0.32$. After that, the wave turns over. In the mean time, the interface develops large curvature and 


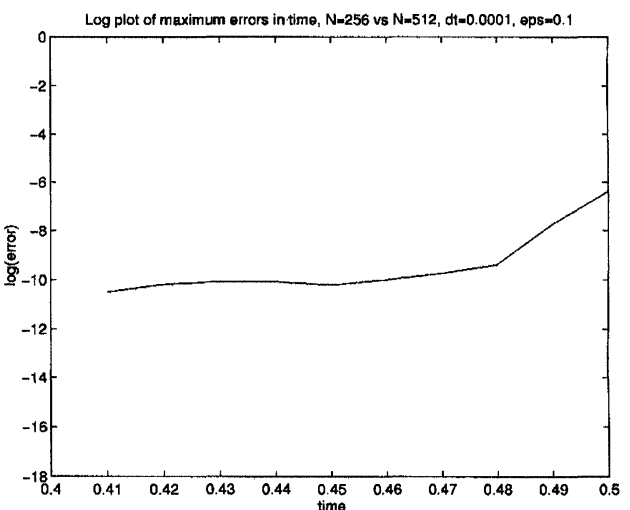

(a)

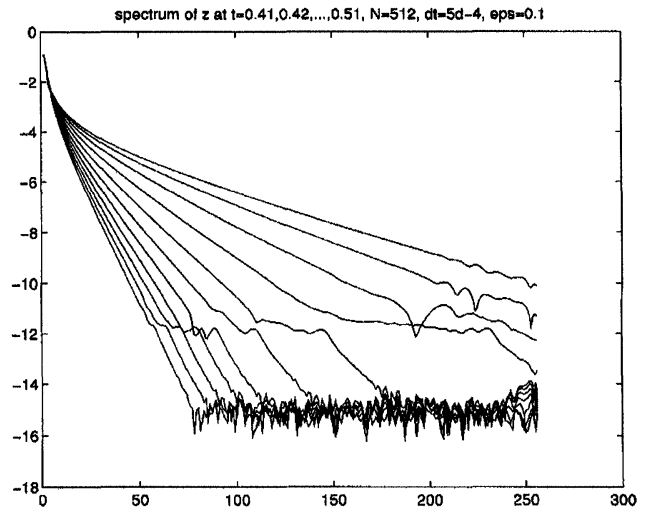

(b)

FIG. 7.

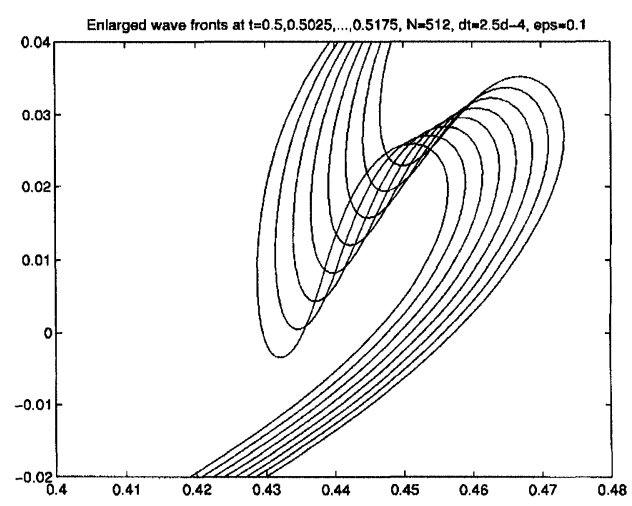

(a)

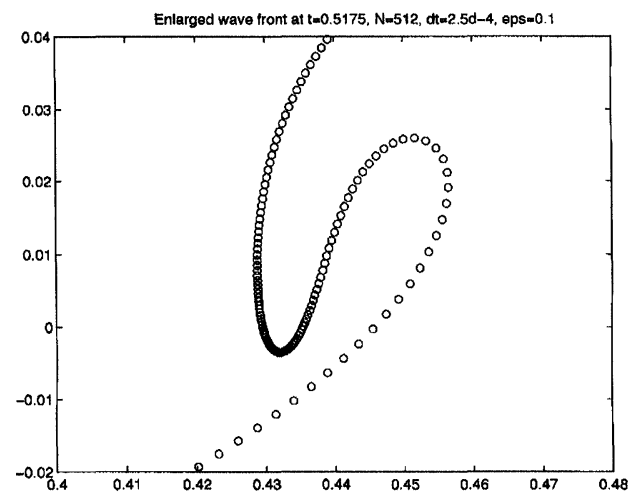

(b)

FIG. 8 .

requires more refined numerical resolution. With $N=256$, we can compute up to $t=0.5$ with six digits of accuracy in the interface positions; see Figure 7a. But in order to compute very close to the time of wave breaking, we need to increase our resolution to $N=512$ or larger. Of course, beyond $t=0.32$ where the interface becomes vertical, our convergence result will cease to be valid since it violates our condition (11) in Theorem 1. But one can see that our numerical calculations remain robust even after condition (11) is violated. In this highly nonlinear regime, the numerical filtering in our algorithm becomes crucial to remain stable in time. We plot the Fourier spectrum of the interface positions in Figure $7 \mathrm{~b}$. It is clear that the numerical round-off errors are kept small even in the fully nonlinear regime of our calculations.

In Figure 8a, we plot the enlarged version of the wave fronts from $t=0.5$ to 0.5175 when the wave is close to breaking. It is evident that the wave will break in finite time. In Figure $8 \mathrm{~b}$, we illustrate the number of computational particles near the wave front at the final time of our calculations. We can see that the interface is still well resolved and more particles are clustered near the head of the wave front. This demonstrates the self-adaptive nature of the boundary integral method. As the interface is close to breaking, the curvature increases very rapidly. In Figure 9a, we plot a sequence of curvatures from $t=0.48$ to $t=0.515$. It is more illustrative if we plot curvature as a function of arclength. This gives us a better idea 


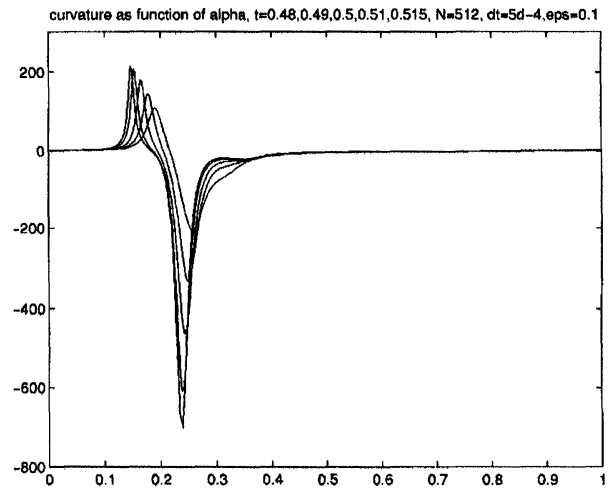

(a)

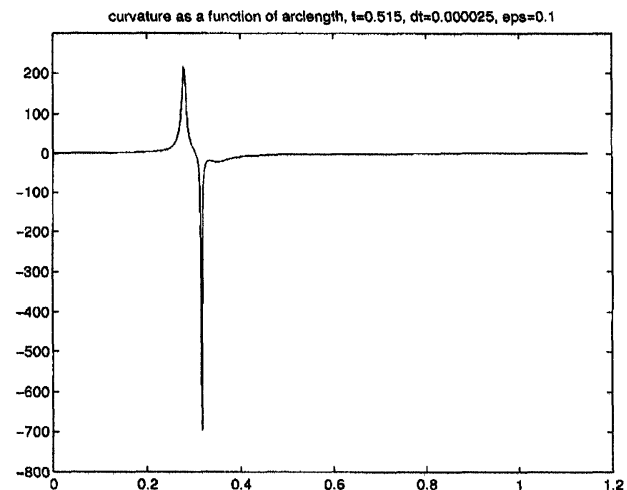

(b)

FIG. 9.

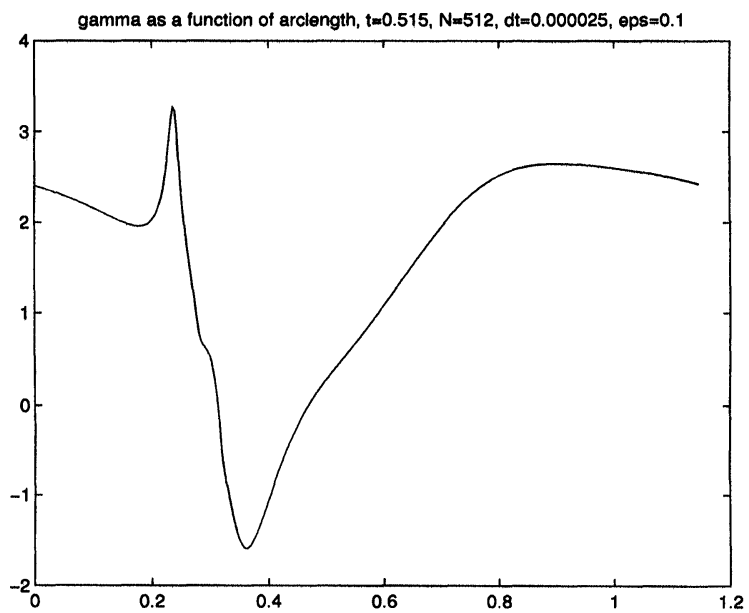

FIG. 10.

how rapidly the curvature changes in a small neighborhood of the wave front; see Figure $9 \mathrm{~b}$. We plot the vortex sheet strength $\gamma$ in Figure 10.

Finally, we would like to emphasize that our initial condition is 1-periodic and our gravity coefficient $g$ is taken to be 9.8. The initial vortex sheet strength is perturbed around 1 . This is responsible for obtaining a breaking wave with a relatively small perturbation of the equilibrium solution in short time. If we change to a $2 \pi$-periodic initial condition and set $g=1$ as in other water wave calculations, then we can obtain a similar wave breaking for larger perturbation from the equilibrum solution. In Figure 11, we present a calculation using the initial condition

$$
\begin{aligned}
& x(\alpha, 0)=\alpha, \quad y(\alpha, 0)=0.6 \cos (\alpha), \\
& \gamma(\alpha, 0)=1.0+0.6 \sin (\alpha) .
\end{aligned}
$$

And the gravity coefficient is set to 1 . Then the wave is going to break around $t=3.75$; see Figure 11. For comparison, we also present the previous calculation at $t=0.5175$ in Figure 12. 


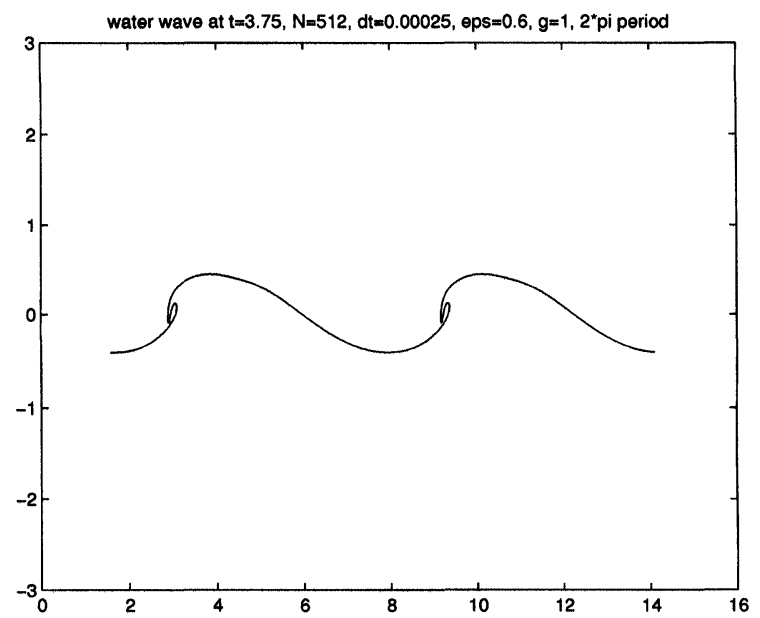

FIG. 11.

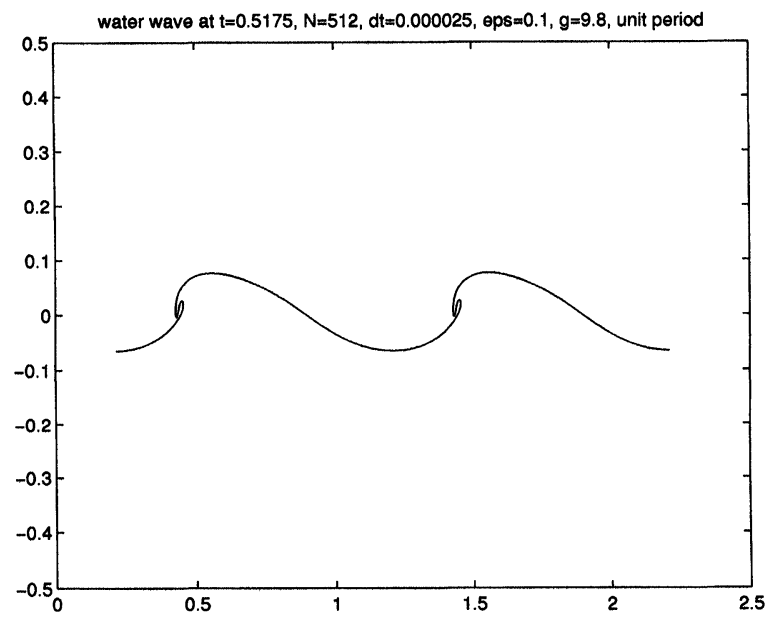

FIG. 12.

It would be interesting to see how the breaking wave calculation is affected by surface tension and whether it prevents the curvature from growing without bound. This would require us to use the reformulation introduced in [HLS] to remove the time step restriction. This will be reported elsewhere.

\section{Appendix.}

Proof of Lemma 1. From the assumption, we know that $\rho$ is $C^{2}$ on $-\pi \leq \xi \leq \pi$, $\rho( \pm \pi)=0$, and $\rho$ is even. Recall that $\frac{h N}{2}=\pi$. We define $R(\xi)=\xi \rho(\xi)$. Then $R$ is $C^{2}$, $R( \pm \pi)=0$, and $R$ is odd. Thus, $R^{\prime}$ is even, and $R^{\prime}(-\pi)=R^{\prime}(\pi)$. Note that if we extend $R$ periodically with period $2 \pi$, then $R$ is $C^{1}$ everywhere and $C^{2}$ except for possible jumps in $R^{\prime \prime}$ at $\xi= \pm \pi$, etc.

Now assume that $f$ is smooth and $w \in l^{2}$. Then we have

$$
(f \cdot w)(x)=\sum_{k_{1}, K_{2} \in l} \hat{f}_{k_{1}} \hat{w}_{k_{2}} e^{i\left(k_{1}+k_{2}\right) x}
$$


with $I=\left\{-\frac{N}{2}+1 \leq l \leq \frac{N}{2}\right\}$. Let $k=k_{1}+k_{2}$ and consider one term in the following two cases:

(1) Suppose $k=k_{1}+k_{2} \in I$. Then we get

$$
D_{h} e^{i k x}=i k \rho(k h) e^{i k x}=\frac{i}{h} R(k h) e^{i k x} .
$$

Now use Taylor's theorem with the remainder

$$
R(k h)=R\left(k_{2} h\right)+R^{\prime}\left(k_{2} h\right) k_{1} h+\int_{0}^{1}(1-t) R^{\prime \prime}\left(k_{2} h+t k_{1} h\right) d t \cdot k_{1}^{2} h^{2} .
$$

Thus, we obtain

$$
D_{h} e^{i k x}=e^{i k_{1} x}\left(D_{h} e^{i k_{2} x}\right)+\left(i k_{1} e^{i k x_{1}}\right) Q\left(k_{2} h\right) e^{i k_{2} x}+h \cdot C_{k_{1} k_{2}} k_{1}^{2} e^{i k x},
$$

where $Q(x)=R^{\prime}(x)$ and $C_{k_{1} k_{2}}$ is bounded independent of $k_{1}, k_{2}$.

(2) Suppose $k_{1}+k_{2}>\frac{N}{2}$. Let $k=k_{1}+k_{2}$ and $\tilde{k}=k-N=k_{1}+k_{2}-N$. Then

$$
D_{h} e^{i\left(k_{1}+k_{2}\right) x}=i \tilde{k} \rho(\tilde{k} h) e^{i \tilde{k} x}=\frac{i}{h} R(\tilde{k} h) e^{i \tilde{k} x} .
$$

Now extend $R$ periodically past $\pi=\frac{N}{2} h$. As noted earlier, $R$ is $C^{1}$ and $C^{2}$ except for $R^{\prime \prime}$ at $\pi$. The Taylor formula is still valid, and the same argument works!

$$
R(\tilde{k} h)=R(k h)=R\left(k_{2} h\right)+\cdots .
$$

Of course, if $k_{1}+k_{2}<-\frac{N}{2}$, we can argue similarly.

The first two terms in the expression above give the two main terms in (23). We still need to estimate the error term. We have to show that it is bounded in $l^{2}$ by $C h\|w\|_{l^{2}}$. Omitting $h$, the coefficient of $e^{i k x}, k \in I$, is

$$
\sum_{k_{1}+k_{2}=k(\bmod N)} C_{k_{1} k_{2}} k_{1}^{2} \hat{f}_{k_{1}} \hat{w}_{k_{2}} .
$$

We can think of this as a discrete integral operator, taking a function of $k_{2}$ to a function of $k$. Using the discrete version of Young's inequality, we conclude that the operator is bounded on $l^{2}$ provided that

$$
\sum_{k_{1} \in I} k_{1}^{2}\left|\hat{f}_{k_{1}}\right| \leq C
$$

which holds since $f \in C^{3}$. This completes the proof of Lemma 1.

Proof of Lemma 2. Recall that $\hat{w}_{k}^{p}=\rho(k h) \hat{w}_{k}$. We have

$$
F\left(w_{i}\right) \equiv \sum_{(j-i) \text { odd }} f\left(\alpha_{i}, \alpha_{j}\right) w_{j}^{p} 2 h=\sum_{l=1}^{N / 2} f\left(\alpha_{i}, \alpha_{i+2 l-1}\right) w_{i+2 l-1}^{p} 2 h .
$$

Denote by $\hat{f}_{k}(\alpha)$ the Fourier coefficient of $f(\alpha, \beta)$ as a function of $\beta$. Further, we can write $F$ as

$$
\begin{aligned}
F\left(w_{i}\right) & =2 h \sum_{l=1}^{N / 2} \sum_{k_{1}, k_{2} \in I} \hat{f}_{k_{1}}\left(\alpha_{i}\right) \hat{w}_{k_{2}} \rho\left(k_{2} h\right) e^{i\left(k_{1}+k_{2}\right) \alpha_{i+2 l-1}} \\
& =2 h \sum_{k_{1}, k_{2} \in l} \hat{f}_{k_{1}}\left(\alpha_{i}\right) \hat{w}_{k_{2}} \rho\left(k_{2} h\right) e^{i\left(k_{1}+k_{2}\right) \alpha_{i-1}} \sum_{l=1}^{N / 2} e^{i\left(k_{1}+k_{2}\right) \alpha_{2 l}} .
\end{aligned}
$$


It is easy to see that if $k_{1}+k_{2} \neq 0 \bmod \left(\frac{N}{2}\right)$, then we have

$$
\sum_{l=1}^{N / 2} e^{i\left(k_{1}+k_{2}\right) \alpha_{2 l}}=0 .
$$

Therefore,

$$
\begin{aligned}
F\left(w_{i}\right)= & \pi \sum_{\substack{k_{1}, k_{2} \in I \\
k_{1}+k_{2}=0 \bmod (N / 2)}} \hat{f}_{k_{1}}\left(\alpha_{i}\right) \hat{w}_{k_{2}} \rho\left(k_{2} h\right) e^{i\left(k_{1}+k_{2}\right) \alpha_{i-1}} \\
= & \pi \sum_{k_{1} \in I} \hat{f}_{k_{1}}\left(\alpha_{i}\right) \hat{w}_{-k_{1}} \rho\left(-k_{1} h\right) \\
& +\pi \sum_{k_{1}=0}^{N / 2} \hat{f}_{k_{1}}\left(\alpha_{i}\right) \hat{w}_{N / 2-k_{1}} \rho\left(\left(N / 2-k_{1}\right) h\right) e^{i \alpha_{i-1} N / 2} \\
& +\pi \sum_{k_{1}=-N / 2+1}^{-1} \hat{f}_{k_{1}}\left(\alpha_{i}\right) \hat{w}_{-N / 2-k_{1}} \rho\left(\left(-\frac{N}{2}-k_{1}\right) h\right) e^{i \alpha_{i-1} N / 2} \\
\equiv & \mathrm{I}+\mathrm{II}+\mathrm{III} .
\end{aligned}
$$

Recall that $f(\alpha, \beta)$ is a smooth function in both variables. So $\hat{f}_{k_{1}}\left(\alpha_{i}\right)$ is a smooth function in $\alpha_{i}$. Moreover, $\hat{f}_{k_{1}}\left(\alpha_{i}\right)$ and its derivative in $\alpha_{i}$ decay like $O\left(k_{1}^{-m}\right)$, where $m$ is the number of continuous derivatives of $f$ with respect to the second variable. Therefore, we have

$$
\left|D_{h}^{l} I\left(w_{i}\right)\right| \leq C\|w\|_{l^{2}} \text { for } 0 \leq l \leq m
$$

and

$$
\left|I\left(S_{h}^{l} w_{i}\right)\right| \leq C \sum_{k_{1} \in I}\left|\hat{f}_{k_{1}}\right|\left|k_{1}\right|^{l}\left|\hat{w}_{k_{1}}\right| \leq C\|w\|_{l^{2}} \quad \text { for } 0 \leq l \leq m-1
$$

We conclude that $I=A_{-(m-1)}(w)$.

For the term II, we note that $\hat{f}_{k_{1}}\left(\alpha_{i}\right) e^{-i \alpha_{i-1} N / 2}$ is not a smooth function in $\alpha_{i}$. However, since $\rho(\pi)=0$, we get

$$
\left|\rho\left(\pi-k_{1} h\right)\right|=\left|\rho(\pi)-\rho^{\prime}(\pi) k_{1} h+\cdots\right| \leq C k_{1} h .
$$

As a result, we obtain

$$
\begin{aligned}
\left|\mathrm{II}\left(w_{i}\right)\right| & \leq \pi \sum_{k_{1}=1}^{N / 2}\left|\hat{f}_{k_{1}}\left(\alpha_{i}\right)\right|\left|\hat{w}_{N / 2-k_{1}} \rho\left(\left(\frac{N}{2}-k_{1}\right) h\right)\right| \\
& \leq C h \sum_{k_{1}=1}^{N / 2} \frac{\left|\hat{w}_{N / 2-k_{1}}\right|}{k_{1}^{m-1}} \leq C h\|w\|_{l^{2}},
\end{aligned}
$$

since $m>2$. This proves $\mathrm{II}\left(w_{i}\right)=h A_{0}(w)$. Clearly, we have

$$
\left\|D_{h} \mathrm{II}\left(w_{i}\right)\right\|_{l^{2}} \leq C\|w\|_{l^{2}} \text { and }\left\|\mathrm{II}\left(S_{h} w_{i}\right)\right\|_{l^{2}} \leq C\|w\|_{l^{2}} .
$$

Similarly, we can prove III $=h A_{0}(w)$ and, consequently, III $=A_{-1}$. If, in addition, we have $\rho^{\prime}(\pi)=0$, then $\left|\rho\left(\pi-k_{1} h\right)\right| \leq C k_{1}^{2} h^{2}$. Then we have II $=h^{2} A_{0}(w)$. This proves Lemma 2 . 
Proof of Lemma 3. We can express $H_{h} f$ as a convolution,

$$
H_{h} f_{i}=\frac{2 h}{\pi} \sum_{(j-i) \text { odd }} \frac{f_{j}}{\alpha_{i}-\alpha_{j}}=(K * f)\left(\alpha_{i}\right),
$$

where

$$
K\left(\alpha_{j}\right)= \begin{cases}\frac{2}{\pi \alpha_{j}} & \text { if } j \text { is odd } \\ 0 & \text { if } j \text { is even }\end{cases}
$$

and $*$ is the discrete convolution,

$$
K * f\left(\alpha_{i}\right)=\sum_{j} K\left(\alpha_{i}-\alpha_{j}\right) f_{j} h
$$

Thus, we have

$$
\left(H_{\hat{h}} f\right)_{k}=\hat{K}_{k} \hat{f}_{k}
$$

with

$$
\hat{K}_{k}=\frac{2 h}{\pi} \sum_{l=-\infty}^{\infty} \frac{e^{-i(2 l+1) k h}}{(2 l+1) h}=\frac{2}{\pi} \sum_{l=-\infty}^{\infty} \frac{e^{-i(2 l+1) k h}}{(2 l+1)}
$$

We claim that

$$
\frac{2}{\pi i} \sum_{l=-\infty}^{\infty} \frac{e^{i(2 l+1) \xi}}{(2 l+1)}=\operatorname{sgn}(\xi), \quad|\xi|<\pi .
$$

We extend $\operatorname{sgn}(\xi)$ periodically outside the interval $|\xi|<\pi$, with period $2 \pi$. To prove (A5) is the same as proving that $\operatorname{sgn}(\xi)$ has the Fourier expension given by the left-hand side of (A5). Let $F(\xi)=\operatorname{sgn}(\xi)$. The Fourier coefficients of $F$ are given by

$$
\hat{F}_{k}=\frac{1}{2 \pi} \int_{-\pi}^{\pi} F(\xi) e^{-i k \xi} d \xi=\frac{1}{\pi k i}\left(1-(-1)^{k}\right) \quad \text { for } k \neq 0
$$

and for $k=0$, we have $\hat{F}_{0}=0$. This proves that

$$
\hat{F}_{k}=\left\{\begin{array}{cl}
\frac{2}{\pi i(2 l+1)} & \text { if } k=2 l+1, \\
0 & \text { if } k=2 l .
\end{array}\right.
$$

Then by the classical result on convergence of Fourier series for piecewise smooth functions,

$$
F(\xi)=\operatorname{sgn}(\xi)=\sum_{k} \hat{F}_{k} e^{i k \xi}=\frac{2}{\pi i} \sum_{l=-\infty}^{\infty} \frac{e^{i(2 l+1) \xi}}{(2 l+1)}
$$

for $0<|\xi|<\pi$ on which $F(\xi)$ is smooth. This proves (A5). Using (A5), we easily obtain that

$$
\hat{K}_{k}=\frac{2}{\pi} \sum_{l=-\infty}^{\infty} \frac{e^{-i(2 l+1) k h}}{(2 l+1)}=i \operatorname{sgn}(-k h)=-i \operatorname{sgn}(k)
$$


Hence,

$$
H_{\hat{h}} f_{k}=-i \operatorname{sgn}(k) \hat{f}_{k} \text { for }|k|<\frac{N}{2},
$$

where we have defined $\operatorname{sgn}(0)=0$. From (A9), we get

$$
H_{h}\left(H_{h}\right)=(-i)^{2} I=-I
$$

on functions $f$ with $\hat{f}_{0}=0, \hat{f}_{N / 2}=0$. We now proceed to evaluate the symbol of

$$
\begin{aligned}
|D| f_{i} & \equiv \frac{1}{\pi} \sum_{(j-i) \text { odd }} \frac{\left(f_{i}-f_{j}\right)}{\left(\alpha_{i}-\alpha_{j}\right)^{2}} 2 h \\
& =\frac{1}{\pi} f_{i} \sum_{(j-i) \text { odd }} \frac{2 h}{\left(\alpha_{i}-\alpha_{j}\right)^{2}}-\frac{2 h}{\pi} \sum_{(j-i) \text { odd }} \frac{f_{j}}{\left(\alpha_{i}-\alpha_{j}\right)^{2}} .
\end{aligned}
$$

Define

$$
G\left(\alpha_{j}\right)= \begin{cases}\frac{2}{\pi \alpha_{j}^{2}} & \text { if } j \text { is odd } \\ 0 & \text { if } j \text { is even. }\end{cases}
$$

Then we can write

$$
|D| f_{i}=\frac{2}{\pi h} f_{i} \sum_{l=-\infty}^{\infty} \frac{1}{(2 l+1)^{2}}-G * f\left(\alpha_{i}\right) .
$$

Thus, we obtain

$$
|D| f_{\hat{k}}=\left(\frac{2}{\pi h} \sum_{l=-\infty}^{\infty} \frac{1}{(2 l+1)^{2}}-\hat{G}_{k}\right) \hat{f}_{k},
$$

where

$$
\hat{G}_{k}=\frac{2 h}{\pi} \sum_{l=-\infty}^{\infty} \frac{e^{-i(2 l+1) k h}}{[(2 l+1) h]^{2}}=\frac{2}{\pi h} \sum_{l=-\infty}^{\infty} \frac{e^{-i(2 l+1) k h}}{(2 l+1)^{2}} .
$$

Using the identity

$$
\sum_{l=-\infty}^{\infty} \frac{1}{(2 l+1)^{2}}=\frac{\pi^{2}}{4}
$$

we obtain

$$
|D| f_{\hat{k}}=\left(\frac{\pi}{2 h}-\frac{2}{\pi h} \sum_{l=-\infty}^{\infty} \frac{e^{-i(2 l+1) k h}}{(2 l+1)^{2}}\right) \hat{f}_{k}
$$

We claim that

$$
\frac{2}{\pi} \sum_{l=-\infty}^{\infty} \frac{e^{i(2 l+1) \xi}}{(2 l+1)^{2}}=\frac{\pi}{2}-|\xi|, \quad|\xi|<\pi
$$


As before, to verify (A16), we first extend $P(\xi) \equiv \frac{\pi}{2}-|\xi|$ periodically out the interval $|\xi|<\pi$ with period $2 \pi$, then compute its Fourier coefficients. They are given by

$$
\hat{P}_{k}=\frac{1}{2 \pi} \int_{-\pi}^{\pi} P(\xi) e^{-i k \xi} d \xi=\frac{1-(-1)^{k}}{\pi k^{2}} \text { if } k \neq 0 .
$$

And clearly $\hat{P}_{0}=0$. This proves (A16) since $P(\xi)$ is piecewise smooth and continuous at $\xi=0$. It follows from (A16) that

$$
\frac{\pi}{2 h}-\frac{2}{\pi h} \sum_{l=-\infty}^{\infty} \frac{e^{-i(2 l+1) k h}}{(2 l+1)^{2}}=\frac{|-k h|}{h}=|k| .
$$

That is,

$$
|D| f_{\hat{k}}=|k| \hat{f}_{k}
$$

On the other hand,

$$
\left.\widehat{H_{h}\left(S_{h} f_{k}\right.}\right)=-i \operatorname{sgn}(k) \cdot(i k) \hat{f}_{k}=|k| \hat{f}_{k} \quad \text { for }|k h|<\pi .
$$

Therefore, we obtain

$$
H_{h}\left(S_{h} f_{i}\right)=\frac{1}{\pi} \sum_{(j-i) \text { odd }} \frac{f_{i}-f_{j}}{\left(\alpha_{i}-\alpha_{j}\right)^{2}} 2 h
$$

for functions $f$ with $\hat{f}_{0}=0$ and $\hat{f}_{N / 2}=0$. And it is an easy matter to check that

$$
H_{h}\left(S_{h}\right)=S_{h} H_{h},
$$

since each operator is multiplication in transformed space. This completes the proof of Lemma 3.

Proof of Lemma 4. By definition, we have from (27) that

$$
\dot{E}_{i}^{L}=\frac{1}{2 \pi i} \sum_{(j-i) \text { odd }} \frac{\dot{\gamma}_{j}}{z\left(\alpha_{i}\right)^{p}-z\left(\alpha_{j}\right)^{p}} 2 h-\frac{1}{2 \pi i} \sum_{(j-i) \text { odd }} \frac{\gamma\left(\alpha_{j}\right)\left(\dot{z}_{i}^{p}-\dot{z}_{j}^{p}\right)}{\left(z\left(\alpha_{i}\right)^{p}-z\left(\alpha_{j}\right)^{p}\right)^{2}} 2 h .
$$

Using the Taylor expansion, we have

$$
\frac{1}{z\left(\alpha_{i}\right)-z\left(\alpha_{j}\right)}=\frac{1}{z_{\alpha}\left(\alpha_{i}\right)\left(\alpha_{i}-\alpha_{j}\right)}+f\left(\alpha_{i}, \alpha_{j}\right)
$$

and

$$
\begin{aligned}
\frac{\gamma\left(\alpha_{j}\right)}{\left(z\left(\alpha_{i}\right)-z\left(\alpha_{j}\right)\right)^{2}}= & \frac{\gamma\left(\alpha_{i}\right)}{z_{\alpha}^{2}\left(\alpha_{i}\right)\left(\alpha_{i}-\alpha_{j}\right)^{2}} \\
& +\frac{q\left(\alpha_{i}\right)}{\left(\alpha_{i}-\alpha_{j}\right)}+g\left(\alpha_{i}, \alpha_{j}\right),
\end{aligned}
$$

where $f\left(\alpha_{i}, \alpha_{j}\right)$ and $g\left(\alpha_{i}, \alpha_{j}\right)$ are smooth functions of $\alpha_{i}$ and $\alpha_{j}$, and

$$
q\left(\alpha_{i}\right)=\frac{\gamma_{\alpha}\left(\alpha_{i}\right) z_{\alpha}\left(\alpha_{i}\right)+\gamma\left(\alpha_{i}\right) z_{\alpha \alpha}\left(\alpha_{i}\right)}{z_{\alpha}^{3}\left(\alpha_{i}\right)} .
$$


Note that $z\left(\alpha_{i}\right)^{p}=z\left(\alpha_{i}\right)+C\left(\alpha_{i}\right) h^{r}$ for some smooth $C(\alpha)$. We have

$$
z\left(\alpha_{i}\right)^{p}-z\left(\alpha_{j}\right)^{p}=z\left(\alpha_{i}\right)-z\left(\alpha_{j}\right)+O\left(h^{r}\right)=\left(z\left(\alpha_{i}\right)-z\left(\alpha_{j}\right)\right)\left(1+O\left(h^{r}\right)\right) \text { for } i \neq j .
$$

Thus, we get

$$
\frac{\gamma\left(\alpha_{j}\right)}{\left(z\left(\alpha_{i}\right)^{p}-z\left(\alpha_{j}\right)^{p}\right)^{2}}=\left(1+O\left(h^{r}\right)\right) \frac{\gamma\left(\alpha_{j}\right)}{\left(z\left(\alpha_{i}\right)-z\left(\alpha_{j}\right)\right)^{2}}
$$

Therefore, it follows from Lemmas 2 and 3 that

$$
\begin{aligned}
& \frac{1}{2 \pi i} \sum_{(j-i) \text { odd }} \frac{\gamma\left(\alpha_{j}\right)\left(\dot{z}_{i}^{p}-\dot{z}_{j}^{p}\right)}{\left(z\left(\alpha_{i}\right)^{p}-z\left(\alpha_{j}\right)^{p}\right)^{2}} 2 h=\left(1+O\left(h^{r}\right)\right) \frac{1}{2 \pi i} \sum_{(j-i) \text { odd }} \frac{\gamma\left(\alpha_{j}\right)\left(\dot{z}_{i}^{p}-\dot{z}_{j}^{p}\right)}{\left(z\left(\alpha_{i}\right)-z\left(\alpha_{j}\right)\right)^{2}} 2 h \\
& =\left(1+O\left(h^{r}\right)\right)\left(\frac{\gamma\left(\alpha_{i}\right)}{2 i z_{\alpha}^{2}\left(\alpha_{i}\right)} H_{h} S_{h}\left(\dot{z}^{p}\right)-\frac{q\left(\alpha_{i}\right)}{2 i} H_{h}\left(\dot{z}^{p}\right)+A_{-1}(\dot{z})\right) \\
& =\frac{\gamma\left(\alpha_{i}\right)}{2 i z_{\alpha}^{2}\left(\alpha_{i}\right)} H_{h} D_{h}(\dot{z})-\frac{q\left(\alpha_{i}\right)}{2 i} H_{h}\left(\dot{z}^{p}\right)+A_{-1}(\dot{z}),
\end{aligned}
$$

since $S_{h}\left(\dot{z}^{p}\right)=D_{h}(\dot{z})$ and $r \geq 2$. Similarly, we have

$$
\frac{1}{2 \pi i} \sum_{(j-i) \text { odd }} \frac{\dot{\gamma}_{j}}{z\left(\alpha_{i}\right)^{p}-z\left(\alpha_{j}\right)^{p}} 2 h=\frac{1}{2 i z_{\alpha}\left(\alpha_{i}\right)} H_{h}(\dot{\gamma})+R_{h}(\dot{\gamma})+A_{-1}(\dot{\gamma}),
$$

where $R_{h}$ is the discrete "smoothing" operator defined by (24), and the $A_{-1}$ term corresponds to the error between $z$ and $z^{p}$. This completes the proof of Lemma 4 .

Proof of Lemma 5. First we state a result for the corresponding continuous problem. Let

$$
K \gamma(\alpha)=\operatorname{Re}\left(\frac{1}{2 \pi i} \int \frac{\gamma\left(\alpha^{\prime}\right) z_{\alpha}(\alpha) d \alpha^{\prime}}{z(\alpha)-z\left(\alpha^{\prime}\right)}\right)
$$

with $z(\alpha, t)$ smooth and $z_{\alpha} \neq 0$. It is easy to see that the kernel $K$ defined above is adjoint to the kernel $\tilde{K}$ corresponding to the dipole strength formulation

$$
\tilde{K} \gamma(\alpha)=\operatorname{Re}\left(\frac{1}{2 \pi i} \int \frac{\gamma\left(\alpha^{\prime}\right) z_{\alpha}\left(\alpha^{\prime}\right) d \alpha^{\prime}}{z(\alpha)-z\left(\alpha^{\prime}\right)}\right) \text {. }
$$

It has been proved in [BMO] that $\left(\frac{1}{2} I+\tilde{K}\right)$ is invertible and the inverse is bounded. Therefore, we have $\left(\frac{1}{2} I+K\right)$ is invertible and

$$
\left\|\left(\frac{1}{2} I+K\right)^{-1}\right\|_{L^{2}} \leq C
$$

We now show that $\left(\frac{1}{2} I+K_{h}\right)$ is invertible. We first consider the case when

$$
\bar{K}_{h} \dot{\gamma}=\sum_{(j-i) \text { odd }} \operatorname{Re}\left\{\frac{1}{2 \pi i}\left[\frac{D_{h} z\left(\alpha_{i}\right)}{z\left(\alpha_{i}\right)-z\left(\alpha_{j}\right)}\right]\right\} \dot{\gamma}_{j} 2 h
$$

For any discrete $l^{2}$ function $\gamma$, we define $\phi$ by

$$
\phi_{i}=\frac{1}{2} \gamma_{i}+\bar{K}_{h} \gamma_{i}
$$


We will show that $\phi=0$ implies that $\gamma=0$. This implies the invertibility of $\left(\frac{1}{2} I+\bar{K}_{h}\right)$. Let $I_{i}=\left[\alpha_{i}-h, \alpha_{i}+h\right]$. If $\alpha \in I_{i}$ with $i$ odd, we partition the real line as the union of $I_{i}$ with $i$ even. We define

$$
\begin{gathered}
K^{o}\left(\alpha, \alpha^{\prime}\right)=\operatorname{Re}\left(\frac{1}{2 \pi i} \frac{D_{h} z\left(\alpha_{i}\right)}{z\left(\alpha_{i}\right)-z\left(\alpha_{j}\right)}\right) \text { if } \alpha \in I_{i}, \quad \alpha^{\prime} \in I_{j} \quad \text { for } j \text { even; } \\
K^{o}\left(\alpha, \alpha^{\prime}\right)=0 \text { otherwise. }
\end{gathered}
$$

If $\alpha \in I_{i}$ with $i$ even, we partition the real line as the union of $I_{i}$ with $i$ odd. Similarly, we define $K^{e}\left(\alpha, \alpha^{\prime}\right)$ as the piecewise constant kernel. Moreover, we define $f^{o}(\alpha)=\gamma_{i}$, and $\phi^{o}(\alpha)=\phi_{i}$ if $\alpha \in I_{i}$ and $i$ is odd. Similarly, we define $f^{e}(\alpha)=\gamma_{i}$, and $\phi^{e}(\alpha)=\phi_{i}$ if $\alpha \in I_{i}$ and $i$ is even. Note that because of the alternating-point quadrature rule, when $i$ is odd, $\bar{K}_{h} \gamma_{i}$ uses only those $\gamma_{j}$ with $j$ even, and vice versa. Thus, for odd-valued index $i$, we have

$$
\frac{1}{2} \gamma_{i}+\bar{K}_{h} \gamma_{i}=\frac{1}{2} f^{o}\left(\alpha_{i}\right)+\int K^{o}\left(\alpha_{i}, \alpha^{\prime}\right) f^{e}\left(\alpha^{\prime}\right) d \alpha^{\prime},
$$

and for even-valued index $i$

$$
\frac{1}{2} \gamma_{i}+\bar{K}_{h} \gamma_{i}=\frac{1}{2} f^{e}\left(\alpha_{i}\right)+\int K^{e}\left(\alpha_{i}, \alpha^{\prime}\right) f^{o}\left(\alpha^{\prime}\right) d \alpha^{\prime} .
$$

We first consider the case $\alpha \in I_{i}$ with $i$ odd. We have

$$
\int K^{o}\left(\alpha, \alpha^{\prime}\right) f^{e}\left(\alpha^{\prime}\right) d \alpha^{\prime}=\sum_{(j-i) \text { odd }} \int_{\alpha^{\prime} \in I_{j}} \operatorname{Re}\left\{\frac{1}{2 \pi i}\left[\frac{D_{h} z\left(\alpha_{i}\right)}{z\left(\alpha_{i}\right)-z\left(\alpha_{j}\right)}\right]\right\} f^{e}\left(\alpha^{\prime}\right) d \alpha^{\prime}
$$

and

$$
\operatorname{Re}\left(\frac{1}{2 \pi i} \int \frac{z_{\alpha}(\alpha) f^{e}\left(\alpha^{\prime}\right)}{z(\alpha)-z\left(\alpha^{\prime}\right)} d \alpha^{\prime}\right)=\sum_{(j-i) \text { odd }} \int_{\alpha^{\prime} \in I_{j}} \operatorname{Re}\left\{\frac{1}{2 \pi i}\left[\frac{z_{\alpha}(\alpha)}{z(\alpha)-z\left(\alpha^{\prime}\right)}\right]\right\} f^{e}\left(\alpha^{\prime}\right) d \alpha^{\prime} .
$$

Therefore, for $\alpha \in I_{i}$ with $i$ odd, we can write

$$
\begin{aligned}
\int K^{o}\left(\alpha_{i}, \alpha^{\prime}\right) f^{e}\left(\alpha^{\prime}\right) d \alpha^{\prime}= & \operatorname{Re}\left(\frac{1}{2 \pi i} \int \frac{z_{\alpha}(\alpha) f^{e}\left(\alpha^{\prime}\right)}{z(\alpha)-z\left(\alpha^{\prime}\right)} d \alpha^{\prime}\right) \\
& +\sum_{(j-i) \text { odd }} \int_{\alpha^{\prime} \in I_{j}} \operatorname{Re}\left\{\frac{1}{2 \pi i}\left[\frac{D_{h} z\left(\alpha_{i}\right)}{z\left(\alpha_{i}\right)-z\left(\alpha_{j}\right)}-\frac{z_{\alpha}(\alpha)}{z(\alpha)-z\left(\alpha^{\prime}\right)}\right]\right\} \\
& \cdot f^{e}\left(\alpha^{\prime}\right) d \alpha^{\prime} .
\end{aligned}
$$

Note that $D_{h} z\left(\alpha_{i}\right)=z_{\alpha}\left(\alpha_{i}\right)+O\left(h^{m}\right)$. Let

$$
Q\left(\alpha, \alpha^{\prime}\right)=\operatorname{Re}\left(\frac{z_{\alpha}(\alpha)}{z(\alpha)-z\left(\alpha^{\prime}\right)} \frac{1}{2 \pi i}\right)=\operatorname{Re}\left(\frac{1}{2 \pi i\left(\alpha-\alpha^{\prime}\right)}+G\left(\alpha, \alpha^{\prime}\right)\right)=\operatorname{Re}\left\{G\left(\alpha, \alpha^{\prime}\right)\right\},
$$

where $G\left(\alpha, \alpha^{\prime}\right)$ is smooth in $\alpha$ and $\alpha^{\prime}$. This implies that $Q\left(\alpha, \alpha^{\prime}\right)$ is smooth. Thus,

$$
\begin{aligned}
Q\left(\alpha_{i}, \alpha_{j}\right)-Q\left(\alpha, \alpha^{\prime}\right) & =Q_{\alpha}\left(\alpha_{i}+\eta_{i}, \alpha_{j}\right)\left(\alpha_{i}-\alpha\right)+Q_{\alpha^{\prime}}\left(\alpha_{i}+\eta_{i}, \alpha_{j}+\xi_{j}\right)\left(\alpha_{j}-\alpha^{\prime}\right) \\
& =h F\left(\alpha_{i}, \alpha_{j}, \alpha, \alpha^{\prime}\right)
\end{aligned}
$$

for some bounded function $F$. Therefore, we obtain

$$
\int K^{o}\left(\alpha, \alpha^{\prime}\right) f^{e}\left(\alpha^{\prime}\right) d \alpha^{\prime}=\operatorname{Re}\left(\frac{1}{2 \pi i} \int \frac{z_{\alpha}(\alpha) f\left(\alpha^{\prime}\right)}{z(\alpha)-z\left(\alpha^{\prime}\right)} d \alpha^{\prime}\right)+h \sum_{(j-i) \text { odd }} \tilde{F}\left(\alpha_{i}, \alpha_{j}, \alpha\right) f^{e}\left(\alpha_{j}\right) h
$$


for some bounded function $\tilde{F}$. Now, for $\alpha \in I_{i}$ with $i$ odd, the equation

$$
\frac{1}{2} \gamma_{i}+\bar{K}_{h} \gamma_{i}=\phi_{i}
$$

is equivalent to

$$
\frac{1}{2} f^{o}+K f^{e}+h L_{e} f^{e}=\phi^{o}
$$

where $L_{e}$ is a bounded linear operator generated from $\tilde{F}$. Similarly, we can show that for $\alpha \in I_{i}$ with $i$ even, (A18) is equivalent to

$$
\frac{1}{2} f^{e}+k f^{o}+h L_{o} f^{o}=\phi^{e},
$$

where $L_{o}$ is a bounded linear operator. Now we add and subtract the equations for $f^{e}$ and $f^{o}$ and we obtain the equations for $f^{e}+f^{o}$ and $f^{e}-f^{o}$, respectively as follows:

$$
\frac{1}{2}\left(f^{o}+f^{e}\right)+K\left(f^{o}+f^{e}\right)=\phi^{o}+\phi^{e}-h L_{o} f^{o}-h L_{e} f^{e}
$$

and

$$
\frac{1}{2}\left(f^{o}-f^{e}\right)-K\left(f^{o}-f^{e}\right)=\phi^{o}-\phi^{e}+h L_{o} f^{o}-h L_{e} f^{e} .
$$

Since the eigenvalues of $K$ are all strictly less than $\frac{1}{2}$ in absolute values [BMO], the same is true for the operator $(-K)$. Therefore, the Neumann series of $\left(\frac{1}{2} I-K\right)$ converges. Hence, both $\left(\frac{1}{2} I+K\right)$ and $\left(\frac{1}{2} I-K\right)$ are invertible. As a result, we obtain

$$
\left\|f^{o}+f^{e}\right\|_{L^{2}} \leq C\left(\left\|\phi^{o}+\phi^{e}\right\|_{L^{2}}+h\|\gamma\|_{l^{2}}\right)
$$

and

$$
\left\|f^{o}-f^{e}\right\|_{L^{2}} \leq C\left(\left\|\phi^{o}-\phi^{e}\right\|_{L^{2}}+h\|\gamma\|_{l^{2}}\right) .
$$

From this, we easily deduce that

$$
\left\|\gamma_{i}+\gamma_{i+1}\right\|_{l^{2}} \leq C\left(\|\phi\|_{l^{2}}+h\|\gamma\|_{l^{2}}\right)
$$

and

$$
\left\|\gamma_{i}-\gamma_{i+1}\right\|_{l^{2}} \leq C\left(\|\phi\|_{l^{2}}+h\|\gamma\|_{l^{2}}\right)
$$

which implies that

$$
\|\gamma\|_{l^{2}} \leq \tilde{C}\|\phi\|_{l^{2}}
$$

where $\tilde{C}$ is a constant independent of $h$. This proves that $\left(\frac{1}{2} I+\bar{K}_{h}\right)$ is invertible since $\phi=0$ implies $\gamma=0$. The above estimate shows that the inverse operator is bounded.

Furthermore, we note that

$$
\frac{D_{h} z\left(\alpha_{i}\right)}{z\left(\alpha_{i}\right)-z\left(\alpha_{j}\right)}-\frac{D_{h} z\left(\alpha_{i}\right)}{z\left(\alpha_{i}\right)^{p}-z\left(\alpha_{j}\right)^{p}}=h^{m} O\left(\frac{1}{\left(\alpha_{i}-\alpha_{j}\right)^{2}}\right)=h L,
$$

where $L$ is a bounded operator. Thus, for $h$ small, $\left(\frac{1}{2} I+K_{h}\right)$ is invertible and its inverse is bounded independent of $h$, where $K_{h}$ is defined by (56). This completes the proof of Lemma 5 . 
Acknowledgements. The first author is grateful for the hospitality of the Courant Institute and the M.S.R.I. during his sabbatical year. The second and third authors wish to thank I.A.S. for its hospitality.

\section{REFERENCES}

[And] C. Anderson, A vortex method for flows with slight density variations, J. Comput. Phys., 61 (1985), pp. 417-444.

[Bak] G. BAKER, Generalized vortex methods for free-surface flows, in Waves on Fluid Interfaces, R. Meyer, ed., University of Wisconsin Press, Madison, WI, 1983, pp. 53-81.

[BMO] G. BAKER, D. MEIRON, AND S. ORsZAG, Generalized methods for free surface flow problems, J. Fluid Mech., 123 (1982), pp. 477-501.

[BN] G. BAKER AND A. NACHBIN, Stable Methods for Vortex Sheet Motion with Surface Tension, preprint, 1992.

[BS] G. BAKER AND M. SHELleY, On the connection between thin vortex layers and vortex sheets, J. Fluid Mech., 215 (1990), pp. 161-194.

[BHL1] J. T. BEALE, T. HOU, AND J. LOWENGRUB, Growth rates for the linear motion of fluid interfaces away from equilibrium, Comm. Pure Appl. Math., 46 (1993), pp. 1269-1301.

[BHL2] - The Stability of Two-Fluid Flows with Surface Tension, Part 1: Growth Rates for the Linear Motion far From Equilibrium; Part 2: Convergence of Suitably Modified Vortex Methods, manuscript.

[BHL3] - On the Stability and Convergence of Suitably Modified Vortex Methods for Hele-Shaw Flows, manuscript.

[BHL4] - On the well-posedness of two fluid interfacial flows with surface tension, in Proceedings of NATO Advanced Workshop on Singularities in Fluid, Plasma, and Optics, Crete, Greece, July 1992, Russel Caflisch, ed., pp. 11-38.

[BHL5] J. T. BEALE, T. Hou, J. LowENGRUB, AND M. SHelley, Spatial and temporal stability issues for interfacial flows with surface tension, J. Math. Comput. Modeling, 20 (1994), pp. 1-27.

[Bir] G. Birkhoff, Helmholtz and Taylor instability, Proc. Sympos. Appl. Math., 13 (1962), pp. 55-76.

[CL] R. CAFLISCH AND J. LOWENGRUB, Convergence of the vortex method for vortex sheets, SIAM J. Numer. Anal., 26 (1989), pp. 1060-1080.

[Craig] W. CRAIG, An existence theory for water waves and the Bousinesq and the Korteweg-de Vries scaling limits, Comm. Partial Differential Equations, 10 (1985), pp. 787-1003.

[CS] W. CRAIG AND C. SUlem, Numerical simulation of gravity waves, J. Comput. Phys., 108 (1993), pp. 73-83.

[DB] G. DAlquist AND A. BJork, Numerical Methods, Prentice-Hall, Englewood Cliffs, NJ, 1974.

[Do] J. W. DolD, An efficient surface-integral algorithm applied to unsteady gravity waves, J. Comput. Phys., 103 (1992), pp. 90-115.

[DY] D. G. DOMMERMUTH AND D. K. P. YUE, A high order spectral method for the study of nonlinear gravity waves, J. Fluid Mech., 184 (1987), pp. 267-88.

[FR] J. D. FENTON AND M. M. RIENECKER, A Fourier method for solving nonlinear water-wave problems: Application to solitary-wave interactions, J. Fluid Mech., 118 (1982), pp. 411-443.

[GAS] M. Glozman, Y. Agnon, AND M. STiassnie, High-order formulation of the water-wave problem, Phys. Rev. D, 66 (1993), pp. 347-367.

[GO] D. GotTLIEB AND S. A. ORZAG, Numerical analysis of spectral methods: Theory and applications, CBMSNSF Regional Conference Series in Applied Mathematics 26, Society for Industrial and Applied Mathematics, Philadelphia, PA, 1977.

[HLK] T. HOU, J. LOWENGRUB, AND R. KRASNY, Convergence of a point vortex method for vortex sheets, SIAM J. Numer. Anal., 28 (1991), pp. 308-320.

[HLS] T. HOU, J. LOWENGRUB, AND M. SHELLEY, Removing the stiffness from interfacial flows with surface tension, J. Comput. Phys., 114 (1994), pp. 312-338.

[Kerr] R. M. KeRR, Simulation of Rayleigh-Taylor flows using vortex blobs, J. Comput. Phys., 76 (1988), pp. 48-84.

[Kra1] R. KRASNY, Desingularization of periodic vortex sheet rollup, J. Comput. Phys., 65 (1986), p. $292-313$.

[Kra2] - On the singularity formation in a vortex sheet and the point vortex approximation, J. Fluid Mech., 167 (1986), pp. 65-93.

[LC] M. S. LONGUET-HigGins AND E. D. CoKelET, The deformation of steep surface waves on water, I. A numerical method of computation, Proc. Roy. Soc. London Ser. A, 350 (1976), pp. 1-26.

[Moor] D. W. Moore, On the point vortex method, SIAM J. Sci. Statist. Comput., 2 (1981), pp. 65-84.

[NMP] A. L. New, P. MCIvER, AND D. H. PEREgrine, Computations of overturning waves, J. Fluid Mech., 150 (1985), pp. 233-251.

[Per] D. H. Peregrine, Breaking waves on beaches, Ann. Rev. Fluid Mech., 15 (1983), pp. 149-178.

[Po] C. PozRIKIDIS, Boundary Integral and Singularity Methods for Linearized Viscous Flow, Cambridge University Press, London, 1992. 
[Pull] D. Pullin, Numerical studies of surface tension effects in nonlinear Kelvin-Helmholtz and Rayleigh-Taylor instabilities, J. Fluid Mech., 119 (1982), pp. 507-532.

[RS] R. H. RANGEL AND W. A. SiRIGNANO, Nonlinear growth of Kelvin-Helmholtz instability: Effect of surface tension and density ratio, Phys. Fluids, 31 (1988), pp. 1845-1855.

[Rob] A. J. ROBERTS, A stable and accurate numerical method to calculate the motion of a sharp interface between fluids, IMA J. Appl. Math., 31 (1983), pp. 13-35.

[Ros] L. RosENHEAD, The point vortex approximation of a vortex sheet, Proc. Roy. Soc. London Ser. A, 134 (1932), pp. 170-192.

[SF] L. W. SchwarTZ AND J. D. Fenton, Strongly nonlinear waves, Ann. Rev. Fluid Mech., 14 (1982), pp. 39-60.

[Shel] M. SHELLEY, A study of singularity formation in vortex sheet motion by a spectrally accurate method, J. Fluid Mech., 244 (1992), pp. 493-526.

[SI] A. SIDI AND M. ISRAELI, Quadrature methods for periodic singular and weakly singular Fredholm integral equations, J. Sci. Comput., 3 (1988), pp. 201-231.

[SS] M. STIASSNIE AND L. SHEMER, On modifications of the Zakharov equation for surface gravity waves, J. Fluid Mech., 143 (1984), pp. 47-67.

[Str] G. STRANG, Accurate partial differential methods II. Nonlinear problems, Numer. Math., 6 (1964), pp. 37-46.

[Tad] E. TADMOR, Stability analysis of finite difference, pseudo-spectral and Fourier-Galerkin approximations, SIAM Rev., 29 (1987), pp. 525-555.

[Tay] G. I. TAYLOR, The instability of liquid surfaces when accelerated in a direction perpendicular to their planes, Proc. Roy. Soc. London Ser. A, 201 (1950), pp. 192-196.

[Tryg] G. TryggVason, Numerical simulations of the Rayleigh Taylor instabilities, J. Comput. Phys., 75 (1988), pp. 253-282.

[VB] T. VINJE AND P. BREvig, Numerical simulation of breaking waves, Adv. Water Resources, 4 (1981), pp. 77-82.

[WBJ] B. J. WEST, K. A. BRUECKNER, AND R. S. JANDA, A new numerical method for surface hydrodynamics, J. Geophys. Res. C, 92 (1987), pp. 11803-11824.

[Ye] R. W. Yeung, Numerical methods in free-surface flows, Ann. Rev. Fluid Mech., 14 (1982), pp. $395-442$. 